\title{
Specker's Parable of the Over-protective Seer: A Road to Contextuality, Nonlocality and Complementarity
}

\author{
Yeong-Cherng Liang* \\ Group of Applied Physics, University of Geneva, CH-1211 Geneva 4, Switzerland and \\ School of Physics, The University of Sydney, Sydney, New South Wales 2006, Australia \\ Robert W. Spekkent \\ Perimeter Institute for Theoretical Physics, 31 Caroline St. N, Waterloo, Ontario, Canada N2L 2 Y5 \\ Howard M. Wisemant \\ ARC Centre for Quantum Computation and Communication Technology, \\ and Centre for Quantum Dynamics, Griffith University, Brisbane, Queensland 4111, Australia
}

(Dated: September 2, 2011)

In 1960, the mathematician Ernst Specker described a simple example of nonclassical correlations, the counterintuitive features of which he dramatized using a parable about a seer who sets an impossible prediction task to his daughter's suitors. We revisit this example here, using it as an entrée to three central concepts in quantum foundations: contextuality, Bell-nonlocality, and complementarity. Specifically, we show that Specker's parable offers a narrative thread that weaves together a large number of results, including: the impossibility of measurement-noncontextual and outcome-deterministic ontological models of quantum theory (the 1967 Kochen-Specker theorem), in particular the recent state-specific pentagram proof of Klyachko; the impossibility of Bell-local models of quantum theory (Bell's theorem), especially the proofs by Mermin and Hardy and extensions thereof; the impossibility of a preparation-noncontextual ontological model of quantum theory; and the existence of triples of positive operator valued measures (POVMs) that can be measured jointly pairwise but not triplewise. Along the way, several novel results are presented, including: a generalization of a theorem by Fine connecting the existence of a joint distribution over outcomes of counterfactual measurements to the existence of a measurement-noncontextual and outcome-deterministic ontological model; a generalization of Klyachko's proof of the KochenSpecker theorem from pentagrams to a family of star polygons; a proof of the Kochen-Specker theorem in the style of Hardy's proof of Bell's theorem (i.e., one that makes use of the failure of the transitivity of implication for counterfactual statements); a categorization of contextual and Bell-nonlocal correlations in terms of frustrated networks; a derivation of a new inequality testing preparation noncontextuality; and lastly, some novel results on the joint measurability of POVMs and the question of whether these can be modeled noncontextually. Finally, we emphasize that Specker's parable of the over-protective seer provides a novel type of foil to quantum theory, challenging us to explain why the particular sort of contextuality and complementarity embodied therein does not arise in a quantum world.

\section{CONTENTS}

I. Introduction
A. The parable of the over-protective seer
B. Contextuality and Complementarity
C. Outline

II. Preliminaries and a formalization of the parable

A. Joint measurability

B. The existence of a joint distribution

C. Measurement-noncontextual ontological models

D. Preparation noncontextuality

E. Justifying outcome determinism

\footnotetext{
* yeongcherng.liang@unige.ch

† rspekkens@perimeterinstitute.ca

‡ h.wiseman@griffith.edu.au
}

III. No-go theorems for measurement-noncontextual and outcome-deterministic models

A. A double-query $n$-box system allowing only adjacent queries

B. Klyachko's proof of the Kochen-Specker theorem

1. Generalization to all odd $n$.

C. A proof of the Kochen-Specker theorem based on the failure of transitivity of implication

IV. No-go theorems for Bell-local models

A. A separated pair of single-query 3-box systems

B. Mermin's proof of Bell's theorem

1. Generalization to all odd $n$

C. Connection to previous work

D. From OS correlations to PR-box correlations

E. Hardy-type no-go theorems for Bell-local 
models

V. Frustrated networks

VI. No-go theorems for preparation-noncontextual models

A. Diachronic pair of single-query 3-box OS correlations

B. Quantum case

C. Justifying preparation noncontextuality by locality

VII. Joint measurability of POVMs

A. Orthogonal spin axes

B. Trine spin axes

C. Generalized-noncontextual models for joint measurements of POVMs

VIII. Concluding Remarks

IX. Acknowledgement

A. Explicit form of OS correlations in the double-query, 3-box system

B. Proof of theorem 6

C. Maximal quantum violation of the $n$-box-set Klyachko-type Kochen-Specker inequality

D. Explicit form of correlations in the separated pair of single-query 3-box systems

E. Maximum quantum violation of the $n$-box-set Bell-Mermin inequality

F. Necessary and sufficient conditions for joint measurability of noisy spin observables

References

\section{INTRODUCTION}

In the field of quantum foundations, the mathematician Ernst Specker is rightly famous for introducing, with co-author Simon Kochen, the concept of a noncontextual hidden variable model and proving that such a model cannot underly quantum theory. This 1967 result, known as the Kochen-Specker theorem [1], continues to be an active subject of research today (see Ref. 2] for a bibliography). One finds precursors to this result in the 1957 work of Gleason [3] and Bell's 1966 review article on hidden variable models (which refers to Gleason's result) [4], but also in a 1960 paper by Specker entitled "The logic of propositions that are not simultaneously decidable" [5] ].

\footnotetext{
1 The 1967 Kochen-Specker theorem [1] improves upon many of these earlier results by making use of a finite set of observables. It
}

This article studied logical features of quantum theory, in particular the question of the consistency of counterfactual propositions concerning the values of observables that are not comeasurable2. One of the points of the paper was to show that it is possible to conceive of an implication relation that is not transitive.

The idea is illustrated with a parable wherein an overprotective seer sets a simple prediction task to his daughter's suitors. The challenge cannot be met because the seer asks the suitors for a noncontextual assignment of values but measures a system for which the statistics are inconsistent with such an assignment. The present article considers the parable anew and seeks to connect it with modern developments in quantum foundations. In particular, we explore the extent to which the sorts of correlations instantiated in the seer's prediction game can be achieved in a quantum world. Although the precise correlations that are required by the seer do not occur in quantum theory, the prediction game is found to be a good pump for quantum intuitions. It leads quite naturally to proofs of nonlocality and contextuality, to a novel kind of complementarity and to the notion of stronger-than-quantum correlations. Indeed, it provides a narrative thread that is able to weave together a great number of important modern results. That so much can be gleaned from this little prediction game is a testament to the depth of Specker's work. We offer this article as a small tribute to him on the occasion of his 90th birthday.

\section{A. The parable of the over-protective seer}

We begin by reproducing Specker's parable of the overprotective seen, with clarifications by us in square brackets:

At the Assyrian School of Prophets in Arba'ilu in the time of King Asarhaddon [(681-669 BCE)], there taught a seer from Nineva. He was a distinguished representative of his faculty (eclipses of the sun and moon) and aside from the heavenly bodies, his interest was almost exclusively in his daughter. His teaching success was limited; the subject proved to be dry and required a previous knowledge of mathematics which

should be noted, however, that Bell's 1964 proof [6] of quantum nonlocality is also a proof of contextuality using only a finite set of observables; unlike the Kochen-Specker proof, it is statespecific, the first example of this kind.

2 Specker did not use the modern term "counterfactual", but instead referred to "infuturabilities", which had been discussed in a scholastic context in connection with the problem of whether God's omniscience extended to knowing the truths of propositions concerning what would have occurred if some event which did not happen had in fact happened.

${ }^{3}$ Our translation is an amalgam of those provided by Stairs [5] and Seevinck [7]. 
was scarcely available. If he did not find the student interest which he desired in class, he did find it elsewhere in overwhelming measure. His daughter had hardly reached a marriageable age when he was flooded with requests for her hand from students and young graduates. And though he did not believe that he would always have her by his side, she was in any case still too young and her suitors in no way worthy. In order that the suitors might convince themselves of their unworthiness, he promised them that she would be wed to the one who could solve a prediction task that was posed to them.

Each suitor was taken before a table on which three little boxes stood in a row, [each of which might or might not contain a gem], and was asked to predict which of the boxes contained a gem and which did not. But no matter how many times they tried, it seemed impossible to succeed in this task. After each suitor had made his prediction, he was ordered by the father to open any two boxes which he had predicted to be both empty or any two boxes which he had predicted to be both full [in accordance with whether he had predicted there to be at most one gem among the three boxes, or at least two gems, respectively]. But it always turned out that one contained a gem and the other one did not, and furthermore the stone was sometimes in the first and sometimes in the second of the boxes that were opened. But how can it be possible, given three boxes, to neither be able to pick out two as empty nor two as full?

The daughter would have remained unmarried until the father's death, if not for the fact that, after the prediction of the son of a prophet [whom she fancied], she quickly opened two boxes herself, one of which had been indicated to be full and the other empty, and the suitor's prediction [for these two boxes] was found, in this case, to be correct. Following the weak protest of her father that he had wanted two other boxes opened, she tried to open the third. But this proved impossible whereupon the father grudgingly admitted that the prediction, being unfalsified, was valid. [The daughter and the suitor were married and lived happily ever after.]

\section{B. Contextuality and Complementarity}

Specker's parable presents us with apparently impossible correlations; as he says "But how can it be possible, given three boxes, to neither be able to pick out two as empty nor two as full?" Indeed, if a suitor reasons classically, then he expects that even if he chooses a configuration of gems at random from among the eight possibilities, it will be the true configuration one time out of eight, and when he opens two boxes he has marked both empty or both full, his prediction will be found to be correct one time out of four. The fact that no suitor manages to succeed after many trials suggests that this reasoning must be flawed and that whichever two boxes are opened, one will be found full and the other empty. Such correlations are contextual in the sense that if one wishes to explain the measurements (opening a box) as revealing a pre-existing property, then one must imagine that the outcome of a measurement (or equivalently, the property that is measured) is context-dependent whether a gem is seen or not in the first box depends on whether that box was opened together with the second or together with the third. The seer's challenge cannot be met by the suitors because he asks them for a noncontextual assignment of outcomes (i.e. a specification of whether a gem will be found or not in each box, independent of which other box is opened with it) but measures a system for which the statistics are inconsistent with such an assignment 4

To imagine a world wherein the parable might occur, Specker must effectively posit the existence of a system that exhibits a particular kind of complementarity: the system must be such that three distinct measurements can implemented upon it, any pair of which can be measured jointly, but where a joint measurement of all three is not possible. To see this, one need only note that if all three binary-outcome measurements could be implemented jointly, some pair would necessarily be found to have correlated outcomes.

We now ask the obvious question: Can the parable be implemented in quantum theory? The reader is urged to pause and give this question some thought before reading on.

There is of course a trivial sense in which the parable can be implemented in a quantum world, namely the same way that it can be implemented in a classical world: through a hidden mechanism under the seer's table and under his control, which inserts and removes gems from the closed boxes at his will. Such a mechanism would allow the seer to enforce complementarity and contextuality "by hand", so to speak. However this is clearly not what Specker had in mind, because had that been the case, the seer would not have been so easily stymied by his daughter's trick, as there would have been no reason why the third box could not have been opened. Rather, the seer seems to be in possession of a set of "magic" boxes that have particular, rather than arbitrary, correlations. Thus in asking the question whether the parable

\footnotetext{
${ }^{4}$ Because the suitors do not fathom this possibility, they are led to interpret their consistent failure to provide a correct prediction as a confirmation of the seer's assessment of their worth. It is in this sense that the seer's task is devised "[i]n order that the suitors might convince themselves of their unworthiness".
} 
can be implemented in quantum theory, we mean: does quantum theory allow for this sort of "magic", which would be truly surprising for a naive suitor familiar only with classical theories, which do not incorporate contextuality and complementarity at a fundamental level?

Certainly, both complementarity and contextuality are required at a fundamental level in quantum theory - measurements that cannot be implemented jointly, and correlations that cannot be explained by noncontextual preexisting properties (see Ref. [8] for a review). But what about the particular correlations of the Specker parable? To get this kind of contextuality, it is necessary to find a situation wherein there are very specific sorts of limitations on joint measurability - there must exist a triple of measurements that can only be implemented jointly in pairs. For projective measurements in quantum theory, this sort of limitation on joint measurability does not occur. The reason is as follows. Two Hermitian operators can be jointly measured if and only they are jointly diagonalizable. But if we have three Hermitian operators $\hat{A}_{1}$, $\hat{A}_{2}$, and $\hat{A}_{3}$, and each pair of operators is jointly diagonalizable, then all three are jointly diagonalizable. This is true for any number of Hermitian operators - one can implement all jointly if and only if one can implement every pair jointly.

Nonetheless, one can imagine modifying the parable in various different ways to obtain something for which an analogue can be found in quantum theory, and these different modifications are the topics of the different sections of our article. In the following we outline each of them in turn.

\section{Outline}

We begin by providing, in Sec. III, a formalization of the original parable as well as some refinements and elaborations thereof, together with definitions of the key concepts. We then present the four different themes inspired by the parable, with an interlude on frustrated networks.

A double-query $n$-box system allowing only adjacent queries (Sec. III). The seer could have a set of $n$ boxes, arranged in a ring, for which only adjacent pairs of boxes can be opened jointly. For $n$ odd, classical intuition leads one to expect that there must exist at least one adjacent pair of boxes that are either both full or both empty, but we can imagine that the seer has a special system wherein, regardless of which adjacent pair of boxes is opened, it is always the case that one is found full and the other empty. The $n=3$ case, which corresponds to the original parable, is exceptional because the adjacent pairs constitute all the pairs. For $n>3$, this is not the case, and so there is no longer any obstacle to finding a set of projective measurements that have the same pattern of joint measurability as these boxes. Indeed, one can find such sets. There are then two ways of trying to obtain a quantum analogue of the new parable.

i) Klyachko's proof of contextuality. Find a quantum state that yields a nonzero probability of anti-correlation for every adjacent pair. When the overall probability is higher than one could account for classically, we arrive at a Klyachko-type proof of quantum contextuality [9].

ii) A new variant of Klyachko's proof of contextuality. Find a quantum state that supports the implication from one outcome to the opposite outcome for every adjacent pair in the ring and that assigns a non-zero probability to the first outcome in the sequence of inferences. In conjunction with the transitivity of implication (a consequence of noncontextuality), and the fact that the ring contains an odd number of boxes, this gives rise to a contradiction, thereby demonstrating the contextuality of quantum theory.

A separated pair of single-query 3-box systems (Sec. IV). One can imagine that the seer's three-box system is such that only a single box (rather than a pair of boxes) can be opened at any given time, but that it is possible to prepare a pair of three-box systems such that by opening a single box on each element of the pair, one reproduces the seer's correlations. Specifically, if the same box is opened on each member of the pair, they are always found to be both full or both empty, while if different boxes are opened on the two systems, one is always found full and the other empty. (Classically, one would expect that some pair of boxes on a given wing are both full or both empty, and by the assumed perfect correlation between the wings, the same pair is similarly configured on the other wing, implying that it is impossible to open different boxes on the two systems and always find anti-correlation rather than correlation.) Here, we are postulating six distinct measurements (three on each wing) only certain pairs of which can be implemented jointly, namely, pairs that have one member from each wing. So again, there is no obstacle to finding a set of projectors having this pattern of joint measurability. There are once again two ways of trying to obtain a quantum analogue of the new parable.

i) Mermin's proof of Bell-nonlocality. Find a quantum state that yields perfect correlation when the same measurement is implemented on the two wings. Demonstrate that the extent to which it can yield anti-correlation when different boxes are opened is greater than is possible in a Bell-local model [10].

ii) Hardy's proof of Bell-nonlocality. Find a chain of choices of measurement, alternating between the two parties, and find a quantum state that yields implications connecting particular outcomes of all but one measurement within this chain. Demonstrate that there is a nonzero probability for the kind of correlation exhibited by the last pair in the chain to be opposite to what one would expect by the transitivity of implication [11].

We also consider generalizations of these nonlocality proofs to rings of $n$ measurements where only adjacent members can be implemented jointly.

Interlude on frustrated networks (Sec. V). Вy representing correlations between binary-valued observables by frustrated networks, we provide a simple cat- 
egorization of some of the contextual and Bell-nonlocal correlations outlined above.

A diachronic pair of single-queries of a 3-box system (Sec. VI). In this case, the seer's three-box system is modified so that only a single box can be opened at any given time, but that it is possible to implement two consecutive measurements in such a way that if the same box is opened at the two times, then the result of the measurement is always reproduced faithfully, while if different boxes are opened at the two times, then the results are always different. In addition, we impose the constraint that no measurement at the second time can yield any information about the choice of the measurement at the first time.

Now it is natural for a suitor to assume that statistical indistinguishability among a set of choices implies that they are equivalent at the level of an ontological model. This assumption is known as preparation noncontextuality [12]. It can be shown that no such preparation-noncontextual model can reproduce the diachronic (two-time) correlations stated above. But in quantum mechanics (which violates preparation noncontextuality [12]), there are sets of measurements for which these correlations can be approximated even though the quantum state after the first measurement reveals no information about the identity of this measurement.

Joint measurability of POVMs (Sec. VII). A final path to a quantum analogue of the overprotective seer (OS) parable is to ignore the counter-intuitive correlations, and rather concentrate on the complementarity exhibited by the three boxes. As discussed above, the pairwise but not triplewise joint measurability of three observables cannot exist in quantum mechanics for traditional (projective) measurements. However, this does not rule out the possibility that there exists a triple of generalized measurements, described by POVMs (positive operator valued measures), that can be jointly measured pairwise but not triplewise. Indeed, we will exhibit two specific examples of such a triple of nonprojective measurements. This thread connects with some recent results on joint measurability of POVMs [13 16]. We demonstrate that this example is not useful for approximating the OS correlations, nor for proving the contextuality of quantum theory.

\section{PRELIMINARIES AND A FORMALIZATION OF THE PARABLE}

\section{A. Joint measurability}

We wish to flesh out the original parable by being more specific about the nature of the correlations posited therein. We shall do this within the context of operational theories. This is natural because the OS parable was originally presented by Specker as a "toy theory" with similarities to quantum theory, not as a scenario that arises within quantum theory. We thus need a unified framework to compare the OS theory both with quantum theory, and with classical theories (i.e. theories without contextuality or complementarity). Also, to make the most of the OS parable we need to embellish the narrative (in a formal way) by adding extra assumptions, and this requires considering measurements and preparations beyond those discussed by Specker. Finally, we note that in the fields of quantum foundations and quantum information, there is currently considerable interest in operational "foil" theories such as Popescu-Rohrlich (PR) boxes [17] and the toy-bit theory [18].

An operational theory is one that specifies the probabilities of each possible outcome $X$ of each possible measurement procedure $M$ given each possible preparation procedure $P$. We denote these probabilities by $p(X \mid M ; P)$. It will be important for the later discussion of contextuality to distinguish between a measurement procedure $M$, which is a specification of a list of instructions of what to do in the laboratory, and an equivalence class $\mathcal{M}$ of measurement procedures, where two procedures are equivalent if they yield the same statistics for all preparation procedures. For instance, the equivalence class associated with a particular measurement procedure $M_{1}$ is

$$
\mathcal{M}_{1} \equiv\left\{M \mid \forall P: p(X \mid M ; P)=p\left(X \mid M_{1} ; P\right)\right\} .
$$

We will refer to this equivalence relation over procedures as operational equivalence. We will refer to the equivalence classes as simply measurements, and denote them by calligraphic font, while the measurement procedures will be denoted by italic font. Similarly, we define equivalence classes of preparation procedures. For instance, the equivalence class associated with a particular preparation procedure $P_{1}$ is

$$
\mathcal{P}_{1} \equiv\left\{P \mid \forall M: p(X \mid M ; P)=p\left(X \mid M ; P_{1}\right)\right\} .
$$

Given that probabilities of outcomes of measurements depend only on the equivalence classes of the preparation and the measurement procedures, we typically condition only on the latter and write $p(X \mid \mathcal{M} ; \mathcal{P})$.

We begin by providing an operational definition of joint measurability 5 . We consider only measurements with a discrete set of outcomes.

Joint measurability of a set of $N$ measurements can be defined (recursively) as follows.

Definition 1 (joint measurability). A set of $N$ measurements $\left\{\mathcal{M}_{1}, \mathcal{M}_{2}, \ldots, \mathcal{M}_{N}\right\}$ is jointly measurable if there exists a measurement $\mathcal{M}$ with the following features: (i) The outcome set of $\mathcal{M}$ is the Cartesian product of the outcome sets of $\left\{\mathcal{M}_{1}, \mathcal{M}_{2}, \ldots, \mathcal{M}_{N}\right\}$ and (ii) the outcome distributions for every joint measurement of a subset $\left\{\mathcal{M}_{s} \mid s \in S\right\} \subset\left\{\mathcal{M}_{1}, \mathcal{M}_{2}, \ldots, \mathcal{M}_{N}\right\}$ are recovered as

\footnotetext{
5 This definition is a generalization of the notion of coexistence of quantum observables provided in Ref. [13].
} 
marginals of the outcome distribution of $\mathcal{M}$ for all preparations $\mathcal{P}$. Denoting a joint measurement of the subset $S$ by $\mathcal{M}_{S}$, and its outcome by $X_{S}$,

$$
\mathcal{M}_{S} \equiv\left\{\mathcal{M}_{s} \mid s \in S\right\}, \quad X_{S} \equiv\left\{X_{s} \mid s \in S\right\}
$$

the condition can be expressed as

$\forall S, \forall \mathcal{P}: p\left(X_{S} \mid \mathcal{M}_{S} ; \mathcal{P}\right)=\sum_{X_{t}: t \notin S} p\left(X_{1}, X_{2}, \ldots, X_{N} \mid \mathcal{M} ; \mathcal{P}\right)$.

Definition 2 ( $n$-tuple-wise joint measurability). A set of measurements $\left\{\mathcal{M}_{1}, \mathcal{M}_{2}, \ldots, \mathcal{M}_{N}\right\}$ is n-tuple-wise jointly measurable if every $n$-element subset (i.e. every $n$-tuple of measurements) is jointly measurable.

Clearly joint measurability of all $n$-tuples implies joint measurability of all $(n-1)$-tuples, but not vice-versa.

Finally, we shall sometimes say that the measurements in a set $\left\{\mathcal{M}_{1}, \mathcal{M}_{2}, \ldots, \mathcal{M}_{N}\right\}$ exhibit complementary if they are not jointly measurable.

We can now be precise about the nature of the correlations in the overprotective seer's prediction game.

Abstracting from the story of boxes and gems, the parable posits that there are three distinct measurement procedures, which we shall denote by $M_{1}, M_{2}$ and $M_{3}$ (corresponding to the choice of box). A key assumption that is not explicit in Specker's description of the prediction game is that these three measurement procedures are not operationally equivalent. That is, for every pair, there is a preparation procedure that distinguishes them, that is, some $P$ such that $p\left(X \mid M_{1} ; P\right) \neq p\left(X \mid M_{2} ; P\right)$. Making this assumption, we see that the game assumes the existence of three distinct equivalence classes of measurement procedures, which we denote by $\mathcal{M}_{1}, \mathcal{M}_{2}$ and $\mathcal{M}_{3}$. Furthermore, it is assumed that these are pairwise jointly measurable. It follows that there exist three joint measurements, which we shall denote by $\mathcal{M}_{12}, \mathcal{M}_{13}$ and $\mathcal{M}_{23}$ and which, by virtue of the definition of joint measurability, must have statistics that reproduce the statistics of $\mathcal{M}_{1}, \mathcal{M}_{2}$ and $\mathcal{M}_{3}$ as marginals. Note that, as the notation suggests, $\mathcal{M}_{12}, \mathcal{M}_{13}$ and $\mathcal{M}_{23}$ correspond to distinct equivalence classes of measurement procedures, a fact that follows from the operational distinguishability of $\mathcal{M}_{1}, \mathcal{M}_{2}$ and $\mathcal{M}_{3}$.

Note also that within the equivalence class of measurement procedures $\mathcal{M}_{1}$, there are procedures $M_{1}^{(2)}$ that involve implementing a joint measurement of $\mathcal{M}_{1}$ and $\mathcal{M}_{2}$ and discarding the outcome of the $\mathcal{M}_{2}$ measurement, and there are procedures $M_{1}^{(3)}$ that involve implementing a joint measurement of $\mathcal{M}_{1}$ and $\mathcal{M}_{3}$ and discarding the outcome of the $\mathcal{M}_{3}$ measurement. Which of these two procedures is implemented may be relevant in a contextual hidden variable model, as we will see.

The seer's trick also requires that there is at least one preparation, call it $\mathcal{P}_{*}$, that yields perfect negative correlations for the joint measurement of any pair of $\mathcal{M}_{1}, \mathcal{M}_{2}$ and $\mathcal{M}_{3}$. Perfect negative correlation for a single joint measurement of $\mathcal{M}_{1}$ and $\mathcal{M}_{2}$ does not imply that one must have equal probability for $X_{1}=0, X_{2}=1$ and $X_{1}=1, X_{2}=0$ (the two ways of achieving perfect negative correlation). However, this equality does follow from demanding perfect negative correlation for all three joint measurements, as we show in Appendix $\mathrm{A}$ Consequently, the correlations are of the form

$$
\begin{aligned}
\forall i \neq j: p\left(X_{i}=0, X_{j}=1 \mid \mathcal{M}_{i, j} ; \mathcal{P}_{*}\right) & =\frac{1}{2} \\
p\left(X_{i}=1, X_{j}=0 \mid \mathcal{M}_{i, j} ; \mathcal{P}_{*}\right) & =\frac{1}{2}
\end{aligned}
$$

We call these the overprotective seer correlations, or $O S$ correlations. Note that it follows from this definition that individual measurements have a uniformly random outcome,

$$
p\left(X_{i}=0 \mid \mathcal{M}_{i} ; \mathcal{P}_{*}\right)=p\left(X_{i}=1 \mid \mathcal{M}_{i} ; \mathcal{P}_{*}\right)=\frac{1}{2} .
$$

\section{B. The existence of a joint distribution}

The question of joint measurability concerns what is physically possible, not what is logically possible. If a physical theory postulates measurements that cannot be jointly implemented, it could still be that there is a joint probability distribution over the outcomes of these measurements that yields each measurement's statistics as a marginal.

Definition 3 (existence of a joint distribution). Consider a set of measurements $\left\{\mathcal{M}_{1}, \mathcal{M}_{2}, \ldots, \mathcal{M}_{N}\right\}$. Let $S$ be a subset of their indices and denote the joint measurement associated with this subset by $\mathcal{M}_{S}$ and its outcome by $X_{S}$, as in Eq. (3). A joint distribution for the measurements $\left\{\mathcal{M}_{1}, \mathcal{M}_{2}, \ldots, \mathcal{M}_{N}\right\}$ is said to exist if there exists a distribution $p\left(X_{1} \ldots X_{N} \mid \mathcal{P}\right)$ for every preparation $\mathcal{P}$ such that for any measurement $\mathcal{M}_{S}$,

$$
p\left(X_{S} \mid \mathcal{M}_{S} ; \mathcal{P}\right)=\sum_{X_{\nu}: \nu \notin S} p\left(X_{1} \ldots X_{N} \mid \mathcal{P}\right) .
$$

It is worth noting that within a given theory, the nonexistence of a joint distribution for some set of measurements implies the physical impossibility of a joint measurement of these. This follows from the fact that if a joint measurement is possible, then there must exist a joint distribution over the outcomes. However, the converse implication need not hold. For instance, there are theories, such as the toy theory of Ref. [18] which postulate the physical impossibility of certain joint measurements, but for which a joint distribution over outcomes (effectively a hidden variable model) does exist.

The feature of the OS correlations that is at the root of their peculiarities is the fact that they do not admit of a joint distribution.

Lemma 4 (no joint distribution for OS correlations). There is no distribution $p\left(X_{1}, X_{2}, X_{3}\right)$ on the three binary 
variables $X_{1}, X_{2}$ and $X_{3}$ such that the marginals over pairs of these are of the form of Eq. (5).

Proof. There are eight valuations of $\left(X_{1}, X_{2}, X_{3}\right)$, of the form $(0,0,0),(0,0,1),(0,1,0), \ldots$ But whichever of these valuations is assigned non-zero probability in $p\left(X_{1}, X_{2}, X_{3}\right)$, one of the three pairs $\left(X_{1}, X_{2}\right),\left(X_{1}, X_{3}\right)$ or $\left(X_{2}, X_{3}\right)$ will have non-zero probability assigned either to the valuation $(0,0)$, "both boxes empty", or to the valuation $(1,1)$ "both boxes full". For this pair, either $p(0,0)>0$ or $p(1,1)>0$, so that perfect anti-correlation is not achieved.

Given the discussion above, this result has immediate (negative) consequences for the possibility of implementing a triplewise joint measurement of $\mathcal{M}_{1}, \mathcal{M}_{2}$ and $\mathcal{M}_{3}$.

Corollary 5. Measurements $\mathcal{M}_{1}, \mathcal{M}_{2}$ and $\mathcal{M}_{3}$ that can be pairwise jointly measured and that achieve the OS correlations of Eq. (5) cannot be triplewise jointly measured.

\section{Measurement-noncontextual ontological models}

In this article, we will make use of the generalized notion of noncontextuality introduced in Ref. [12], which is operational insofar as it is defined for ontological models of any operational theory, not just quantum theory. An ontological model of an operational theory specifies: (i) a set $\Lambda$ of ontic (i.e. real, physical) states $\lambda$; (ii) for each preparation procedure $P$, a distribution $p(\lambda \mid P)$ describing the probability that the ontic state of the system subsequent to the preparation procedure $P$ is $\lambda$; (iii) for each measurement procedure $M$, a response function $p(X \mid M ; \lambda)$ describing the conditional probability of obtaining outcome $X$ given ontic state $\lambda$. Finally, one must recover the statistics of the operational theory as follows:

$$
p(X \mid M ; P)=\sum_{\lambda} p(X \mid M ; \lambda) p(\lambda \mid P) .
$$

Here we have taken $\lambda$ to be a discrete variable for simplicity.

An ontological model is said to be measurementnoncontextual if any two measurement procedures that are operationally equivalent [in the sense of Eq. (10)] are represented similarly in the model:

$$
\begin{aligned}
\forall P: p(X \mid M ; P) & =p\left(X \mid M^{\prime} ; P\right) \\
\Rightarrow \forall \lambda: p(X \mid M ; \lambda) & =p\left(X \mid M^{\prime} ; \lambda\right) .
\end{aligned}
$$

Equivalently, the condition is that the response function for a measurement procedure $M$ depends only on its operational equivalence class $\mathcal{M}$, that is,

$$
p(X \mid M ; \lambda)=p(X \mid \mathcal{M} ; \lambda) .
$$

An ontological model is said to be outcomedeterministic for a measurement procedure $M$ if the outcome is uniquely determined for every ontic state,

$$
\forall \lambda \in \Lambda: p(X \mid M ; \lambda) \in\{0,1\} .
$$

The traditional notion of a noncontextual ontological model of quantum theory incorporated both the assumption of measurement noncontextuality and that of outcome determinism for projective measurements. Here, we will follow Ref. [12] and distinguish these assumptions so as not to conflate issues about determinism with issues about noncontextuality. To avoid terminological confusion, we shall say that an ontological model of quantum theory is traditionally-noncontextual if it is both measurement-noncontextual [in the sense of Eq. (9)] and outcome-deterministic for projective measurements. Any proof of the impossibility of a traditionally-noncontextual model of quantum theory will be called a proof of the Kochen-Specker theorem.

As it turns out, there is a close connection between the existence of a joint distribution and noncontextuality:

Theorem 6. For a given set of measurements, if there exists a measurement-noncontextual and outcomedeterministic ontological model then there exists a joint distribution for their outcomes.

The proof is provided in Appendix B, This is a slight generalization of half of a theorem by Fine [19]. Combining this theorem with the nonexistence of a joint distribution for the OS correlations (lemma 4), we have:

Corollary 7. There is no measurement-noncontextual and outcome-deterministic ontological model of the OS correlations of Eq. (5).

It is also possible to write down inequalities which must be satisfied by the experimental statistics if these are to admit of an explanation in terms of a measurementnoncontextual and outcome-deterministic model. We will call these Kochen-Specker inequalities. For the case of the OS correlations, if we imagine such a model then each box must be either empty or full. Consequently, if we choose a pair of boxes uniformly at random at most two of the three pairs could exhibit anticorrelation, so that the probability of obtaining anticorrelated outcomes is bounded above by $2 / 3$. More precisely, if $p\left(X_{i} \neq X_{i \oplus 1} \mid \mathcal{M}_{i, i \oplus 1} ; \mathcal{P}\right)$ denotes the probability of obtaining anti-correlated outcomes in a joint measurement of $\mathcal{M}_{i}$ and $\mathcal{M}_{i \oplus 1}$, where $\oplus$ denotes addition modulo 3 , then the average probability of success is

$$
R_{3} \equiv \sum_{i=1}^{3} \frac{1}{3} p\left(X_{i} \neq X_{i \oplus 1} \mid \mathcal{M}_{i, i \oplus 1} ; \mathcal{P}\right)
$$

and it satisfies

$$
R_{3} \leq R_{3}^{N C}=\frac{2}{3}
$$

This is a Kochen-Specker inequality.

It is sometimes useful to express Kochen-Specker inequalities in an algebraic form. We define new variables $\bar{X}_{i}=(-1)^{X_{i}}$, so that $\bar{X}_{i}=+1(-1)$ when $X_{i}=0(1)$. 
Using angle brackets to denote averages, we consider the following combination of correlation functions

$$
\mathcal{S}_{3} \equiv\left\langle\bar{X}_{1} \bar{X}_{2}\right\rangle+\left\langle\bar{X}_{2} \bar{X}_{3}\right\rangle+\left\langle\bar{X}_{3} \bar{X}_{1}\right\rangle .
$$

Then the inequality takes the form

$$
\mathcal{S}_{3} \geq \mathcal{S}_{3}^{N C}=-1,
$$

The OS correlations, however, require $\left\langle\bar{X}_{i} \bar{X}_{i \oplus 1}\right\rangle=-1$ for all $i$ and hence $\mathcal{S}_{3}=-3$, clearly violating the bound.

With the correlations in this form, one can also express a proof of the impossibility of an outcome-deterministic noncontextual model in the algebraic manner introduced by Mermin [20]. Assuming that $\bar{X}_{i} \in\{+1,-1\}$ has a value independent of context, the OS correlations require that these values satisfy the following algebraic relations.

$$
\begin{aligned}
\bar{X}_{1} \bar{X}_{2} & =-1, \\
\bar{X}_{1} \bar{X}_{3} & =-1, \\
\bar{X}_{2} \bar{X}_{3} & =-1 .
\end{aligned}
$$

However, these relations cannot be satisfied because the product of the left-hand-sides is $\bar{X}_{1}^{2} \bar{X}_{2}^{2} \bar{X}_{3}^{2}=+1$, while the product of the right-hand-sides is -1 .

Any theory that realizes the OS correlations fails to admit of a measurement-noncontextual and outcomedeterministic ontological model. However, as explained in the introduction, the kind of complementarity one requires to achieve these correlations - three measurements that are pairwise but not triplewise jointly measurable - cannot arise for projective measurements in quantum theory. In Sec. III we turn to the modifications of the parable that do have a counterpart in quantum theory.

\section{Preparation noncontextuality}

The notion of measurement noncontextuality defined in Eq. (9) is motivated by a kind of equivalence principle: in the absence of observable differences between measurement procedures (i.e. differences in their statistics) one should not posit differences in their representations in the ontological model. In Ref. 12] it was argued that the same principle should lead one to an assumption of noncontextuality for preparation procedures. Specifically, an ontological model is said to be preparation noncontextual if any two preparation procedures that are operationally equivalent [in the sense of Eq. (2)] are represented equivalently in the model:

$$
\begin{array}{r}
\forall M: p(X \mid M ; P)=p\left(X \mid M ; P^{\prime}\right) \\
\Rightarrow \forall \lambda: p(\lambda \mid P)=p\left(\lambda \mid P^{\prime}\right) .
\end{array}
$$

Preparation noncontextuality can also be characterized as the condition that the distribution for a preparation procedure $P$ depends only on its operational equivalence class $\mathcal{P}$, that is,

$$
p(\lambda \mid P)=p(\lambda \mid \mathcal{P}) .
$$

Given their similar motivations, someone who endorses measurement noncontextuality ought also to endorse preparation noncontextuality just as enthusiastically. One should endorse both notions or neither. Therefore, it is most natural to ask about the possibility of an ontological model that is both preparation-noncontextual and measurement-noncontextual. We will call such models generalized-noncontextual 6. In this paper, we will consider suitors faced with the seer's prediction problem who are committed to the kind of equivalence principle described above and therefore to generalized noncontextuality.

Inequalities that must be satisfied by the experimental statistics if these are to admit of a generalizednoncontextual model will be called simply noncontextuality inequalities. Note that our terminology distinguishes such inequalities from the Kochen-Specker inequalities of the previous section: Kochen-Specker inequalities express constraints on statistics when one assumes outcome determinism in addition to measurement noncontextuality, while noncontextuality inequalities rely on no such assumption of determinism. An example of a noncontextuality inequality will be provided in Sec. VI

\section{E. Justifying outcome determinism}

Note that a commitment to the kind of equivalence principle described above does not obviously provide any grounds for assuming outcome determinism for measurements, Eq. (11). Thus, faced with the OS correlations and corollary 7 a suitor might simply deny outcome determinism to salvage measurement noncontextuality. For instance, seeing the correlations in the seer's prediction game, a clever suitor might hypothesize that they are explained by the following sort of model. There is an ontic variable that flags when the preparation $\mathcal{P}_{*}$ was implemented and if it was, the measurements $\mathcal{M}_{12}, \mathcal{M}_{13}$ and $\mathcal{M}_{23}$ each generate the outcomes $(0,1)$ and $(1,0)$ uniformly at random. Such an ontological model would violate outcome determinism, but would preserve measurement noncontextuality.

On the other hand, the assumption of outcome determinism can sometimes be shown to be a consequence of preparation noncontextuality. If such a justification is forthcoming, then the OS correlations cannot be explained by any ontological model that is generalizednoncontextual. For instance, in quantum theory, the assumption of outcome determinism for projective measurements can be derived from preparation noncontextuality, as shown in Ref. [12]. Therefore, in quantum theory the conjunction of measurement noncontextuality and outcome determinism for projective measurements - i.e. the assumption of traditional noncontextuality

\footnotetext{
6 In Ref [12], these were called "universally noncontextual."
} 
of an ontological model — is implied by the assumption of generalized noncontextuality and all the no-go theorems for the former are no-go theorems for the latter. In Sec. IIII we will provide proofs of the failure of traditional noncontextuality in quantum theory using a generalization of the OS correlations. Given the result just mentioned, such proofs also demonstrate the failure of generalized noncontextuality.

Much of this article makes statements about correlations that are not found in quantum theory but can easily be imagined to occur in more general operational theories. In such theories, a natural analogue of the notion of a projective measurement can be defined. The question thus arises of whether the assumption of preparation noncontextuality might imply outcome determinism for such measurements for an ontological model of a general operational theory. The question is currently open, but we conjecture that it has a positive answer.

Fortunately, we can still draw some negative conclusions about generalized noncontextuality in operational theories without settling this conjecture. Specifically, in Sec. VI] we will demonstrate how a slight modification of the seer's game yields a set of correlations that fails to admit of a preparation-noncontextual ontological model.

\section{NO-GO THEOREMS FOR MEASUREMENT-NONCONTEXTUAL AND OUTCOME-DETERMINISTIC MODELS}

\section{A. A double-query $n$-box system allowing only adjacent queries}

One way to generalize Specker's parable is to consider $n>3$ boxes, and allow only certain pairs to be opened jointly. In particular, one can imagine the boxes to be arranged in a ring with adjacent pairs being the only ones that can be opened jointly. The resulting pattern of joint measurability can be reproduced in quantum theory because there exist ordered sets of $n>3$ projectors for which adjacent elements commute (where adjacency is determined modulo $n$ ). If $n$ is odd, then for every deterministic and noncontextual assignment of gems to boxes that the suitor might make, there must exist at least one adjacent pair of boxes that are either both full or both empty. Indeed, given any assignment of gems to boxes, if we choose an adjacent pair of boxes uniformly at random, the probability of obtaining anti-correlated outcomes is bounded above by $(n-1) / n$. We then imagine that the seer has a special system such that, regardless of which adjacent pair of boxes is opened, it is always the case that one is found full and the other empty. 7 For these correlations, unlike those described in the original

\footnotetext{
7 Note that for even $n$, one cannot develop an interesting parable because there are assignments of gems to boxes wherein no adjacent pair is both full or both empty.
}

parable, one can find a quantum analogue. Although this analogue does not allow the seer to always defeat the suitor's prediction, the probability of finding perfect anti-correlation between a pair of adjacent boxes can be greater than the success rate of $(n-1) / n$ expected by classical reasoning.

Let us consider this situation more carefully. We are imagining an odd number $n \geq 5$ of measurements, $\left\{\mathcal{M}_{a} \mid a=1, \ldots, n\right\}$, such that for all $a, \mathcal{M}_{a}$ and $\mathcal{M}_{a \oplus 1}$ are jointly measurable by a measurement $\mathcal{M}_{a, a \oplus 1}$ (here $\oplus$ denotes addition modulo $n$ ), and that there is at least one preparation, call it $\mathcal{P}_{*}$, such that the outcomes of all of these pairs of measurements are anti-correlated. By a generalization of the argument provided in Appendix A, the correlations must be of the form

$$
\begin{aligned}
\forall a: p\left(X_{a}=0, X_{a \oplus 1}=1 \mid \mathcal{M}_{a, a \oplus 1} ; \mathcal{P}_{*}\right) & =\frac{1}{2} \\
p\left(X_{a}=1, X_{a \oplus 1}=0 \mid \mathcal{M}_{a, a \oplus 1} ; \mathcal{P}_{*}\right) & =\frac{1}{2} .
\end{aligned}
$$

We will call these the double-query n-box OS correlations.

By an argument analogous to the one proving lemma 4 one can show that there is no joint distribution over all the $X_{a}$ that reproduces these correlations as marginals. It then follows from theorem 6 that there is no measurement-noncontextual and outcome-deterministic ontological model of these correlations.

Indeed, if we choose an adjacent pair of boxes uniformly at random, the probability $R_{n}$ of obtaining anticorrelated outcomes,

$$
R_{n} \equiv \sum_{a=1}^{n} \frac{1}{n} p\left(X_{a} \neq X_{a \oplus 1} \mid \mathcal{M}_{a}, \mathcal{M}_{a \oplus 1} ; \mathcal{P}\right),
$$

is clearly bounded above,

$$
R_{n} \leq 1-\frac{1}{n}
$$

(because at most $n-1$ pairs can be anti-correlated if $n$ is odd). The double-query $n$-box OS correlations yield $R_{n}=1$, maximally violating this Kochen-Specker inequality.

We may equivalently state the restriction as follows. Following the convention established in Sec. IIC, we define $\bar{X}_{a}=(-1)^{X_{a}} \in\{+1,-1\}$. For all measurementnoncontextual and deterministic assignments of the value $\bar{X}_{a}$, at most $n-1$ of the pair-wise products can be -1 , so that:

$$
\mathcal{S}_{n} \equiv\left\langle\bar{X}_{1} \bar{X}_{2}\right\rangle+\left\langle\bar{X}_{2} \bar{X}_{3}\right\rangle+\cdots+\left\langle\bar{X}_{n} \bar{X}_{1}\right\rangle \geq-(n-2),
$$

whereas the double-query $n$-box OS correlations give $\mathcal{S}_{n}=-n$.

Again, a simple algebraic way of manifesting the fact that the double-query $n$-box correlations do not admit of a measurement-noncontextual and outcomedeterministic model is that they require $\bar{X}_{a} \in\{+1,-1\}$ 
such that

$$
\begin{gathered}
\bar{X}_{1} \bar{X}_{2}=-1, \\
\bar{X}_{2} \bar{X}_{3}=-1, \\
\vdots \\
\bar{X}_{n-1} \bar{X}_{n}=-1, \\
\bar{X}_{n} \bar{X}_{1}=-1,
\end{gathered}
$$

but the product of the left-hand-sides is $\bar{X}_{1}^{2} \bar{X}_{2}^{2} \cdots \bar{X}_{n}^{2}=$ +1 , while the product of the right-hand-sides is -1 .

We now consider what values of $R_{n}$ and $\mathcal{S}_{n}$ can be achieved in quantum theory.

\section{B. Klyachko's proof of the Kochen-Specker theorem}

We require $n$ Hermitian observables $\hat{X}_{1}, \ldots, \hat{X}_{n}-$ each having eigenvalues 0 and 1 - associated with the $n$ measurements $\mathcal{M}_{1}, \ldots, \mathcal{M}_{n}$. As discussed in Sec. IB for the specific case of $n=3$, the pairwise commutativity of $\hat{X}_{1}, \hat{X}_{2}$ and $\hat{X}_{3}$ implies their triplewise commutativity and consequently the existence of a triplewise joint measurement and of a measurement-noncontextual and outcome-deterministic model 8

Nonetheless, we can obtain something interesting for odd $n$ greater than 3 . We begin with the case of $n=5$. A no-go theorem of this sort has recently been given by Klyachko [21] (see also Refs. 9] and [22]). The construction is as follows. We consider a quantum system described by a 3-dimensional Hilbert space, and all of the states we consider require only real-valued coefficients in some basis. Thus the system can be visualized in 3dimensional Euclidean space. The observables are projectors $\hat{X}_{a}=\left|l_{a}\right\rangle\left\langle l_{a}\right|$, where the vectors $\left\{\left|l_{a}\right\rangle: a=1, \ldots, 5\right\}$ are of the form

$$
\left|l_{a}\right\rangle=\left(\sin \theta \cos \varphi_{a}, \sin \theta \sin \varphi_{a}, \cos \theta\right),
$$

and $\varphi_{a}=\frac{4 \pi a}{5}$ so that the sequence of vectors forms a pentagram, as in Fig. 1 The angle $\theta$ is chosen such that vectors adjacent in the sequence are orthogonal, $\left\langle l_{a} \mid l_{a \oplus 1}\right\rangle=0$, where $\oplus$ denotes sum modulo 5 . As a result of this orthogonality relation, adjacent observables $\hat{X}_{a}, \hat{X}_{a \oplus 1}$ are indeed jointly measurable. It is clear that such a value of $\theta$ exists because as it varies from 0 to $\frac{\pi}{2}$, the angle between adjacent vectors varies from 0 to $\frac{4 \pi}{5}$. In fact, orthogonality is achieved at $\cos \theta=\frac{1}{\sqrt[4]{5}}$.

Now consider a preparation of the quantum state $\left|\psi_{1}\right\rangle$ corresponding to the vector lying along the symmetry

\footnotetext{
8 Another way to see that the Kochen-Specker inequality (15) cannot be violated in quantum theory is that we can treat $\mathcal{S}_{3}$ as a polynomial of commuting variables, and thus its minimum can be attained by assigning the value to each variable in a noncontextual and deterministic manner.
}

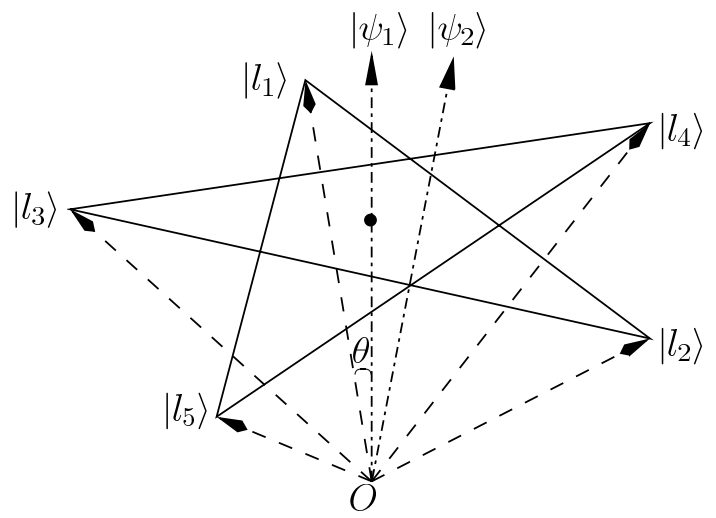

FIG. 1. Quantum states and observables used for Klyachko's proof of contextuality and the proof of contextuality via the failure of transitivity of implication.

axis of the pentagram, such that the angle between it and each of the $\left|l_{a}\right\rangle$ is $\theta$. In a measurement of any adjacent pair of observables $\hat{X}_{a}, \hat{X}_{a \oplus 1}$, either just one of them yields the outcome 1 , in which case the outcomes are anticorrelated, or both yield the outcome 0 . The probability for anti-correlation is $2 \cos ^{2}(\theta)=2 / \sqrt{5}$, which implies that the Kochen-Specker bound of Eq. (21) is violated,

$$
R_{5}=R_{5}^{\text {quantum }}=\frac{2}{\sqrt{5}} \simeq 0.89442 \not \leq \frac{4}{5} .
$$

Equivalently, with the observables $\bar{X}_{a}=2 \hat{X}_{a}-\hat{\mathbf{1}}$, where $\hat{\mathbf{1}}$ is the identity operator, the state $\left|\psi_{1}\right\rangle$ achieves $\mathcal{S}_{5}=$ $5-4 \sqrt{5} \approx-3.9443 \geq-3$.

The value $2 / \sqrt{5}$ is in fact the maximum possible quantum violation of this Kochen-Specker inequality. We show this in Appendix Clwith the help from the converging hierarchy of semidefinite programming (SDP) tools discussed in Ref. 23] [see also Eq. (26) and Eq. (27) below].

Note that unlike the no-coloring proofs of the KochenSpecker theorem, this is a state-specific proof [24, 25] 9 In fact, for 3-dimensional quantum state, this is a statespecific proof that involves the smallest set of vectors $\left\{\left|l_{a}\right\rangle\right\}$ satisfying the orthogonality relation $\left\langle l_{a} \mid l_{a \oplus 1}\right\rangle=$ $0[9] .10$

9 Note also that the claim in Klyachko et al. [9] that the inequality in question provides a "test of arbitrary hidden variables model, context free or not" is mistaken. If the values assigned to observables could be context-dependent, there would be no contradiction.

10 The 2-vector and 3-vector cases are trivial. For the 4 -vector case, note that the orthogonality relation $\left\langle l_{a} \mid l_{a \oplus 1}\right\rangle=0$ implies that some pair of non-adjacent vectors must be collinear which, in turn, implies that all the four projectors $\left|l_{a}\right\rangle\left\langle l_{a}\right|$ must commute, and therefore cannot violate any Kochen-Specker inequality. 


\section{Generalization to all odd $n$.}

One can generalize Klyachko's no-go result to all odd $n$ as follows. Define $n$ observables by the projectors onto vectors $\left\{\left|l_{a}\right\rangle: a=1, \ldots, n\right\}$ defined as in Eq. (24) but with $\varphi_{a}=\frac{n-1}{n} \pi a$ and with $\theta$ chosen such that $\left\langle l_{a} \mid l_{a \oplus 1}\right\rangle=0$, where $\oplus$ denotes sum modulo $n$. This is achieved when $\cos ^{2} \theta=\cos (\pi / n) /(1+\cos (\pi / n))$. This set of $n$ vectors forms what is known as an $\left\{n / \frac{n-1}{2}\right\}$ star polygon [26]. The $\{5 / 2\},\{7 / 3\}$ and $\{9 / 4\}$ star polygons are depicted in Fig. 2] Again, preparing the quantum state on the symmetry axis of the star polygon, the probability of anticorrelation for adjacent observables violates the KochenSpecker bound of Eq. (21) with

$$
R_{n}=R_{n}^{\text {quantum }}=\frac{2 \cos \left(\frac{\pi}{n}\right)}{1+\cos \left(\frac{\pi}{n}\right)} \not 1-\frac{1}{n}
$$

or equivalently, the Kochen-Specker bound of Eq. (22) with

$$
\begin{aligned}
\mathcal{S}_{n}=\mathcal{S}_{n}^{\text {quantum }} & =n-2 n R_{n}^{\text {quantum }} \\
& =n-\frac{4 n \cos \left(\frac{\pi}{n}\right)}{1+\cos \left(\frac{\pi}{n}\right)} \ngtr 2-n .
\end{aligned}
$$

As with the $n=5$ case, these values also represent the strongest possible quantum violation of these KochenSpecker inequalities, as is shown in Appendix C. At large $n$, the quantum probability approaches unity quadratically as

$$
R_{n}^{\text {quantum }} \simeq 1-\frac{\pi^{2}}{4 n^{2}}
$$

in contrast to the linear approach to unity of the KochenSpecker bound.

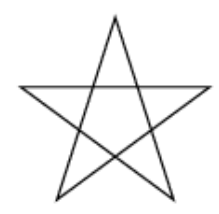

$\left\{\frac{5}{2}\right\}$

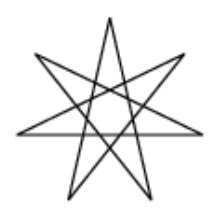

$\left\{\frac{7}{3}\right\}$

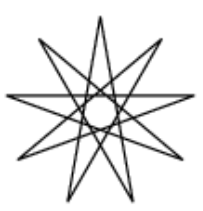

$\left\{\frac{9}{4}\right\}$
FIG. 2. $\left\{n / \frac{n-1}{2}\right\}$ star polygons for $n=5,7$, and 9 .

It is worth emphasizing that by using the quantum correlations for $n$ measurements, the seer can achieve something very close to the ends he achieved in the original parable. Specifically, the seer can construct a prediction game such that suitors who reason classically think the game is fair (i.e. they think it is highly likely that some suitor will win) when in fact it is not (because classical reasoning does not apply and it is actually highly unlikely that any suitor will win).

The prediction game that meets the seer's ends is as follows. The suitor is asked to pick an adjacent pair of boxes that he believes to be both empty or both full and to open those. If his prediction for those two boxes is correct, the suitor wins, otherwise he loses. With what probability will a suitor who reasons classically expect to win? We presume that he knows the seer to be adversarial and so he reasons that the seer has prepared a classical configuration which makes his [the suitor's] task as difficult as possible. He reasons therefore that the configuration is one wherein only one adjacent pair of boxes is both full or both empty (by his classical lights, he knows that there must be at least one such pair for an odd number of boxes). Thus the suitor expects his probability of winning to be the probability that he has guessed correctly which of all the $n$ pairs is the correlated one, times the probability that he has guessed their contents correctly overall, a probability of $1 / 2 n$. In fact, the probability of the suitor's prediction coming true is only of order $1 / n^{2}$ in the quantum scheme described above. Let us say the number of suitors is $l$, assumed large. Then if the seer chooses the number of boxes $n$ such that $n \ll l \ll n^{2}$, the suitors believe it to be very likely that one of them will win when in fact it is very likely that none of them will win.

\section{A proof of the Kochen-Specker theorem based on the failure of transitivity of implication}

Specker's intent in introducing his parable was to demonstrate the logical possibility of a failure of the transitivity of implication. The idea is straightforward. Suppose $s_{1}, s_{2}$ and $s_{3}$ are propositions that assert the presence of a gem in boxes 1,2 and 3 respectively, and $\neg s_{1}$, $\neg s_{2}$ and $\neg s_{3}$ assert their negations. We have $s_{1} \Longrightarrow \neg s_{2}$ (because boxes 1 and 2 are never found both full), and $\neg s_{2} \Longrightarrow s_{3}$ (because boxes 2 and 3 are never found both empty). If implication were transitive, then we could conclude that $s_{1} \Longrightarrow s_{3}$. But in fact we have $s_{1} \Longrightarrow \neg s_{3}$ (because boxes 1 and 3 are never found both full). Therefore, assuming a gem is sometimes found in box 1, transitivity fails.

Specker's 1960 article was framed within the tradition of quantum logic, and although some researchers have proposed that quantum theory might require us to abandon some of the rules of classical logic as rules of rightreasoning (see, for example, Ref. [27]), we will not consider this possibility here. Indeed, if we incorporate the context of a measurement in the propositions, so that we distinguish $s_{1}$, finding a gem in box 1 in the context of measuring box 1 with box 2 , from $s_{1}^{\prime}$, finding a gem in box 1 in the context of measuring box 1 with box 3 , then the transitivity of implication can be salvaged and there is no challenge to classical logic.

Nonetheless, the failure of the transitivity of implication provides another perspective on how to generate no-go results for measurement-noncontextual outcomedeterministic models. In such models, implications among value assignments of observables are necessarily 
transitive because these value assignments do not depend on the context of the measurement. A failure of the transitivity of implication therefore implies the impossibility of such a model.

In the case of the double-query $n$-box OS correlations, if $n$ is odd, the perfect anti-correlations justify the following implications around the ring of boxes,

$$
\begin{aligned}
& X_{1}=1 \Longrightarrow X_{2}=0 \Longrightarrow X_{3}=1 \\
& \Longrightarrow \ldots \Longrightarrow X_{n}=1 \Longrightarrow X_{1}=0 .
\end{aligned}
$$

By the transitivity of implication, we would conclude that $X_{1}=1 \Longrightarrow X_{1}=0$. Given that $X_{1}=1$ is sometimes observed, one has a contradiction. Consequently, the observation of the double-query $n$-box OS correlations implies the impossibility of a measurementnoncontextual outcome-deterministic ontological model.

We now demonstrate the existence of a quantum analogue of this argument in the case of $n=5$. Specifically, we demonstrate that for the set of observables in Klyachko's proof, specified in Eq. (24) and depicted in Fig. 11. there is a quantum state such that

$$
\begin{aligned}
X_{1}=1 \Longrightarrow X_{2}=0 \Longrightarrow X_{3}=1 \\
\Longrightarrow X_{4}=0 \Longrightarrow X_{5}=1 \Longrightarrow X_{1}=0 .
\end{aligned}
$$

First, note that an inference from $X_{a}=1$ to $X_{a \oplus 1}=0$ can be made independently of the quantum state, because for any pair of orthogonal projectors, at most one of them can take the value 1 . However, an inference from $X_{a}=0$ to $X_{a \oplus 1}=1$ is only true for certain quantum states because a pair of projectors may both be assigned the value 0 . To ensure that $X_{a}=0$ implies $X_{a \oplus 1}=1$, we must choose a quantum state that lies in the span of the vectors $\left|l_{a}\right\rangle$ and $\left|l_{a \oplus 1}\right\rangle$ in Hilbert space. This way, the vector orthogonal to this span is assigned value 0 , such that if $\left|l_{a}\right\rangle$ is assigned value $0,\left|l_{a \oplus 1}\right\rangle$ must be assigned the value 1 . Starting with an assignment of $X_{1}=1$, we need to make the $X_{a}=0$ to $X_{a \oplus 1}=1$ inference twice in the pentagram: from $X_{2}=0$ to $X_{3}=1$ and from $X_{4}=0$ to $X_{5}=1$. Consequently, we need a quantum state that lies in the subspace (plane) spanned by $\left|l_{2}\right\rangle$ and $\left|l_{3}\right\rangle$ but also in the subspace spanned by $\left|l_{4}\right\rangle$ and $\left|l_{5}\right\rangle$. Fortunately, these subspaces intersect on a ray (see Fig. 1), and therefore we take the quantum state to be the one associated with that ray, indicated in Fig. 11 as $\left|\psi_{2}\right\rangle$.

Therefore, assuming a preparation of the state $\left|\psi_{2}\right\rangle$, we have the sequence of implications of Eq. (30). By the transitivity of implication, we can conclude that $X_{1}=$ $1 \Longrightarrow X_{1}=0$. Given that $X_{1}=1$ is assigned non-zero probability by $\left|\psi_{2}\right\rangle$, specifically, $p=1-\frac{2}{\sqrt{5}} \approx 0.1056$, we have derived a contradiction from the assumption of the transitivity of implication, and therefore also from the assumption of an ontological model that is measurementnoncontextual and outcome-deterministic for projective measurements (i.e. traditionally-noncontextual) 11 .

\footnotetext{
11 A slightly different way of seeing the contradiction is that tran-
}

This is a proof of the Kochen-Specker theorem which is analogous to Hardy's proof of Bell's theorem, described in Sec. IVE. Interestingly, it is not possible to generalize this type of proof to the case of $n>5$ using a set of vectors that form an $\left\{n / \frac{n-1}{2}\right\}$ star polygon. For instance, in the case of $n=7$, if we start with $X_{1}=1$, in order to make the inference from $X_{2}=0$ to $X_{3}=1$, from $X_{4}=0$ to $X_{5}=1$ and from $X_{6}=0$ to $X_{7}=1$, the quantum state would have to lie in each of the following three subspaces: the one spanned by $\left|l_{2}\right\rangle$ and $\left|l_{3}\right\rangle$, the one spanned by $\left|l_{4}\right\rangle$ and $\left|l_{5}\right\rangle$ and the one spanned by $\left|l_{6}\right\rangle$ and $\left|l_{7}\right\rangle$. But although any pair of these subspaces intersect along a ray, the three do not, so there is no quantum state that does the job.

The state-specific Kochen-Specker proof we have just presented turns out to be related to Clifton's 8-ray Kochen-Specker proof [24]. The latter makes use of the famous 8-vertex subgraph of the original 117-vertex Kochen-Specker proof [1]. Clifton's proof also has an interesting connection with the pre and post-selection effect known as the "three-box paradox" [28], as shown in Ref. [29]. A connection between Klyachko's KochenSpecker proof and the 8-ray proof (as well as Hardy's nonlocality proof) has also been noted previously in Ref. [30].

To see how our proof is related to Clifton's, let us denote the vector orthogonal to the span of $\left|l_{2}\right\rangle$ and $\left|l_{3}\right\rangle$ by $|\chi\rangle$ and the one orthogonal to the span of $\left|l_{4}\right\rangle$ and $\left|l_{5}\right\rangle$ by $\left|\chi^{\prime}\right\rangle$, then the orthogonality relations of the eight vectors $\left\{\left|l_{1}\right\rangle,\left|l_{2}\right\rangle,\left|l_{3}\right\rangle,\left|l_{4}\right\rangle,\left|l_{5}\right\rangle,|\chi\rangle,\left|\chi^{\prime}\right\rangle,\left|\psi_{2}\right\rangle\right\}$ are summarized by the diagram in Fig. 3. (where nodes represent rays and the presence of an edge represents orthogonality). In an outcome-deterministic measurement-noncontextual model, every vector must receive a value 0 or 1 with exactly one member of every orthogonal triple receiving the value 1 , and no more than one member of an orthogonal pair receiving the value 1 .

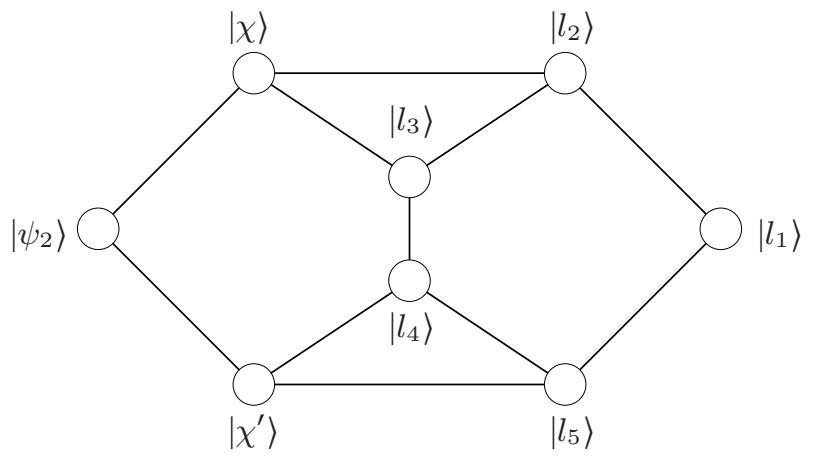

FIG. 3. Clifton's 8-ray state-specific Kochen-Specker proof.

Clifton's proof can then be phrased as follows. Given a preparation of $\left|\psi_{2}\right\rangle$, the vector $\left|\psi_{2}\right\rangle$ (considered as a

sitivity of implication specifies that $X_{1}=1$ implies $X_{5}=1$, whereas by a joint measurement of $X_{1}$ and $X_{5}$, we would infer that $X_{1}=1$ implies $X_{5}=0$. 
measurement outcome) must be assigned the value 1 and the vector $\left|l_{1}\right\rangle$ has a nonzero probability of being assigned the value 1 . We denote the value assigned to vector $|\phi\rangle$ by $v(|\phi\rangle)$. From $v\left(\left|\psi_{2}\right\rangle\right)=1$ we infer $v(|\chi\rangle)=v\left(\left|\chi^{\prime}\right\rangle\right)=$ 0 and from $v\left(\left|l_{1}\right\rangle\right)=1$ (which happens with nonzero probability) we infer $v\left(\left|l_{2}\right\rangle\right)=v\left(\left|l_{5}\right\rangle\right)=0$. One then concludes from $v(|\chi\rangle)=0$ and $v\left(\left|l_{2}\right\rangle\right)=0$ that $v\left(\left|l_{3}\right\rangle\right)=$ 1 , and from $v\left(\left|\chi^{\prime}\right\rangle\right)=0$ and $v\left(\left|l_{5}\right\rangle\right)=0$ that $v\left(\left|l_{4}\right\rangle\right)=1$. However, $v\left(\left|l_{3}\right\rangle\right)=1$ and $v\left(\left|l_{4}\right\rangle\right)=1$ is a contradiction.

This is the standard way of deriving a contradiction for the eight rays in Clifton's proof, however one could equally well use the fact that $v(|\chi\rangle)=v\left(\left|\chi^{\prime}\right\rangle\right)=0$ and $v\left(\left|l_{1}\right\rangle\right)=1$ to justify anticorrelation across every edge around the ring $\left\{\left|l_{1}\right\rangle,\left|l_{2}\right\rangle,\left|l_{3}\right\rangle,\left|l_{4}\right\rangle,\left|l_{5}\right\rangle\right\}$, which is just the proof we have presented above.

\section{NO-GO THEOREMS FOR BELL-LOCAL MODELS}

\section{A. A separated pair of single-query 3-box systems}

In this section, we consider another variation on Specker's parable. The seer has a novel 3-box system which allows only a single box to be opened, rather than two. To distinguish the two types of three-box systems, we call the former a single-query system and the latter a double-query system. We also assume that the seer can prepare a pair of single-query systems that mimic the behavior of the double-query system as follows: if the same box is opened on one system as is opened on the other, one obtains the same result (both are always found to be full, or both empty); if different boxes are opened, then one obtains different results (one is always full and the other empty). For the benefit of skeptical suitors, the seer allows for the queries of the two different systems to be implemented at space-like separation. We imagine that they are transported to different corners of the Assyrian empire: one to Abydos and the other to Babylon. The suitor dispatches two of his trusted classmates, one to each of these two cities, and instructs them to choose a box at random.

We are therefore imagining a situation wherein two observables are measured jointly by first preparing a pair of systems in a perfectly correlated state and measuring one observable on each.

As we will demonstrate below, this version of the Specker parable allows us to establish a simple proof of nonlocality in the same spirit as that presented by Mermin in Ref. [10]. Let us denote the choices made by the two class-mates by $a$ and $b$ respectively, taking values in the set $\{1,2,3\}$, corresponding to the choice of box. Further, we denote the results of box $a$ at Abydos and box $b$ at Babylon, respectively, by $A_{a}$ and $B_{b}$, taking values in $\{1,0\}$ corresponding to the observations \{full, empty $\}$. Then we can express the condition that the outcomes must satisfy in this two-wing version of the Specker parable as

$$
A_{a} \oplus B_{b}=1 \oplus \delta_{a, b},
$$

where $\delta$ denotes the Kronecker delta function. To quantify the extent to which these correlations are realized, let us define $R_{3}$ as the weighted sum, assuming $a$ and $b$ are chosen uniformly at random, of the probability of achieving perfect negative correlation when $a \neq b$, and the probability of achieving perfect correlation when $a=b$. That is,

$$
\begin{aligned}
R_{3} & \equiv \frac{1}{9} \sum_{a, b: b \neq a} p\left(A_{a} \neq B_{b} \mid \mathcal{M}_{a}, \mathcal{M}_{b} ; \mathcal{P}\right) \\
& +\frac{1}{9} \sum_{a, b: b=a} p\left(A_{a}=B_{b} \mid \mathcal{M}_{a}, \mathcal{M}_{b} ; \mathcal{P}\right)
\end{aligned}
$$

where $p\left(A_{a}=B_{b} \mid \mathcal{M}_{a}, \mathcal{M}_{b} ; \mathcal{P}\right)$ refers to the probability of finding $A_{a}=B_{b}$ conditioned on box $a$ being opened at Abydos and box $b$ being opened at Babylon; likewise for $p\left(A_{a} \neq B_{b} \mid \mathcal{M}_{a}, \mathcal{M}_{b} ; \mathcal{P}\right)$.

The OS correlations described in the two-wing Specker parable can be summarized as

$$
\begin{gathered}
\sum_{A_{a}, B_{b}: B_{b}=A_{a}} p\left(A_{a}, B_{b} \mid \mathcal{M}_{a}, \mathcal{M}_{b} ; \mathcal{P}\right)=1 \quad \text { for } a=b, \\
\sum_{A_{a}, B_{b}: B_{b} \neq A_{a}} p\left(A_{a}, B_{b} \mid \mathcal{M}_{a}, \mathcal{M}_{b} ; \mathcal{P}\right)=1 \quad \text { for } a \neq b,
\end{gathered}
$$

The assumption that $\mathcal{M}_{a}$ and $\mathcal{M}_{b}$ are jointly measurable in the sense of definition (1) implies that they must satisfy a condition of no superluminal signaling [17, 31], namely,

$$
\begin{aligned}
& p\left(A_{a} \mid \mathcal{M}_{a} ; \mathcal{P}\right)=\sum_{B_{b}} p\left(A_{a}, B_{b} \mid \mathcal{M}_{a}, \mathcal{M}_{b} ; \mathcal{P}\right) \quad \forall b, \\
& p\left(B_{b} \mid \mathcal{M}_{b} ; \mathcal{P}\right)=\sum_{A_{a}} p\left(A_{a}, B_{b} \mid \mathcal{M}_{a}, \mathcal{M}_{b} ; \mathcal{P}\right) \quad \forall a
\end{aligned}
$$

which asserts that the conditional marginal probabilities $p\left(A_{a} \mid \mathcal{M}_{a} ; \mathcal{P}\right)$ obtained by summing over $B_{b}$ are independent of the choice of the distant measurement procedure $\mathcal{M}_{b}$, and likewise for $p\left(B_{b} \mid \mathcal{M}_{b} ; \mathcal{P}\right)$. It is simple to show, as we do in Appendix D. that by imposing the no-signaling condition, the correlations are constrained to be of the following form:

$$
\begin{aligned}
\forall a \neq b: p\left(0,1 \mid \mathcal{M}_{a}, \mathcal{M}_{b}, \mathcal{P}_{*}\right) & =\frac{1}{2}, \\
p\left(1,0 \mid \mathcal{M}_{a}, \mathcal{M}_{b} ; \mathcal{P}_{*}\right) & =\frac{1}{2}, \\
\forall a=b: p\left(0,0 \mid \mathcal{M}_{a}, \mathcal{M}_{b}, \mathcal{P}_{*}\right) & =\frac{1}{2}, \\
p\left(1,1 \mid \mathcal{M}_{a}, \mathcal{M}_{b} ; \mathcal{P}_{*}\right) & =\frac{1}{2} .
\end{aligned}
$$

We will henceforth call these the nonlocal OS correlations. The winning probability for Eq. (32) is unity for these correlations, i.e.,

$$
R_{3}^{\mathrm{NLOS}}=1
$$


They are the only non-signaling correlations that can win this prediction game deterministically. This implies, in particular, that the nonlocal OS correlations represent an extreme point of the convex set of non-signaling correlations 32], very much like the archetypical PR box 12 correlations [17] for the scenario where $a, b$ only run from 1 to 2 . Although these correlations do not allow for superluminal signaling, they do violate Bell's assumption of local causality [33], as we now demonstrate.

In order to enforce perfect positive correlations when the suitor's two classmates make the same measurement, the Babylonian system must be prepared with an answer for each possible query that matches the answer that the Abydosian system is prepared to provide. It follows that there are deterministic noncontextual hidden variables determining the outcome on the Babylonian system. This step is familiar from Bell's original derivation of his theorem [6]: locality together with the assumption of perfect correlations implies the existence of deterministic noncontextual values for each system. Given such values, it is easy to see 13 that the overall probability of winning the game in a locally causal model is at most $\frac{7}{9}$. That is,

$$
R_{3} \leq R_{3}^{\text {local }}=\frac{7}{9}
$$

This is a Bell inequality. The fact that $R_{3}^{\mathrm{NLOS}}=1$ for the seer's system is a violation of this Bell inequality and a proof that no locally causal model of the nonlocal OS correlations is possible.

The Bell inequality (37) can also be written in terms of the more conventional correlation function, or the socalled two-party correlator $\left\langle\bar{A}_{a} \bar{B}_{b}\right\rangle$, where $\bar{A}_{a}, \bar{B}_{b}$ take on values $\{+1,-1\}$ as usual:

$$
\begin{array}{r}
\left\langle\bar{A}_{a} \bar{B}_{b}\right\rangle=\sum_{\bar{A}_{a}, \bar{B}_{b}: \bar{B}_{b}=\bar{A}_{a}} p\left(\bar{A}_{a}, \bar{B}_{b} \mid \mathcal{M}_{a}, \mathcal{M}_{b} ; \mathcal{P}\right) \\
-\sum_{\bar{A}_{a}, \bar{B}_{b}: \bar{B}_{b} \neq \bar{A}_{a}} p\left(\bar{A}_{a}, \bar{B}_{b} \mid \mathcal{M}_{a}, \mathcal{M}_{b} ; \mathcal{P}\right)
\end{array}
$$

12 The terminology "box" is, in the present circumstances, unfortunate. It refers to a "black-box" (i.e. unexplained - indeed, inexplicable - source of correlations) between two distant parties, just as in our above scenario.

${ }^{13}$ For instance, if the values assigned to the state of the boxes 1,2 , and 3 are $0,0,1$, then we have positive correlation for $(a, b) \in\{(1,1),(2,2),(3,3)\}$ and negative correlation for $(a, b) \in$ $\{(1,3),(3,1),(2,3),(3,2)\}$, in accordance with the nonlocal OS correlations. However, we also have positive correlation for $(a, b) \in\{(1,2),(2,1)\}$ in disagreement with the nonlocal OS correlations. Thus the correlations are correct in only 7 out of 9 cases. Alternative strategies that do not enforce the perfect correlation when $a=b$ cannot do any better. For instance, if all boxes on the left are empty and all boxes on the right are full, then one has 6 out of 6 anti-correlations but 0 out of 3 correlations, leaving a winning probability of $2 / 3$, which is smaller than $7 / 9$.
The two-party correlator is simply the average value of the product of the result in Abydos when box $a$ was chosen, multiplied by the result in Babylon when box $b$ was chosen. Together with the normalization condition $\sum_{\bar{A}_{a}, \bar{B}_{b}} p\left(\bar{A}_{a}, \bar{B}_{b} \mid \mathcal{M}_{a}, \mathcal{M}_{b}, \mathcal{P}\right)=1$, we can now reexpress the winning probability as $R_{3}=\frac{1}{18} \mathcal{S}_{3}+\frac{1}{2}$, where

$$
\mathcal{S}_{3}=\sum_{a}\left\langle\bar{A}_{a} \bar{B}_{a}\right\rangle-\sum_{a \neq b}\left\langle\bar{A}_{a} \bar{B}_{b}\right\rangle .
$$

In these notations, it is again easy to verify that if the variables $\bar{A}_{a}, \bar{B}_{b}$ admit pre-existing values \pm 1 (i.e. are determined by hidden variables), then $\mathcal{S}_{3} \leq 5$ [cf. Eq. (37)] (As is now well-known [19], the same bound also applies to any locally causal model where the values of the variables are determined by stochastic hidden variable models.) Specker's correlations require $\mathcal{S}_{3}=9$, thus clearly violating the Bell-inequality.

\section{B. Mermin's proof of Bell's theorem}

What about correlations allowed in quantum theory? We know from a celebrated theorem by Cleve et al. (Theorem 5.12, Ref. 34]) that there is no quantum strategy that can give unit winning probability. While it is not possible to realize the over-protective seer parable as formulated above, it is nevertheless possible to demonstrate, using quantum mechanics, correlations that approximate the desired correlations better than any locally causal model can. As it turns out, the largest winning probability allowed by quantum theory is (see Appendix $\mathrm{E}$ for details)

$$
R_{3}^{\text {quantum }}=\frac{5}{6}
$$

and hence $\mathcal{S}_{3}^{\text {quantum }}=6$ (which exceed the Bell-local bounds of $\frac{7}{9}$ and 5 respectively). That quantum theory allows such non-trivial correlations can be verified by considering the two-qubit maximally entangled state $\frac{1}{\sqrt{2}}(|0\rangle|0\rangle+|1\rangle|1\rangle)$ (in the $\hat{\sigma}_{z}$ basis) and letting $\bar{A}_{1}, \bar{A}_{2}$, and $\bar{A}_{3}$ be the results of measuring the three Pauli operators equally spaced in the $\hat{z}-\hat{x}$ plane, defined by

$$
\hat{A}_{a}=\cos \frac{2 \pi(a-1)}{3} \hat{\sigma}_{z}+\sin \frac{2 \pi(a-1)}{3} \hat{\sigma}_{x} ;
$$

likewise for the $\hat{B}_{b}$, which are defined identically. Thus quantum mechanics allows us to move towards the extremal non-local correlations in our formulation of the parable. This proof that quantum theory violates Belllocality (Bell's theorem) is in fact the one popularized by David Mermin [10].

\section{Generalization to all odd $n$}

It is straightforward to generalize this new parable to the case of $n$ boxes for all odd $n \geq 5$. Specifically, posit 
a separated pair of $n$-box rings such that if the box that is opened in Abydos is the same as the one opened in Babylon, the outcomes agree, while if the index $a$ of the box opened in Abydos differs by 1 from the index $b$ of the one opened in Babylon, that is, if $b=a \oplus 1$ or $a=$ $b \oplus 1$, then the outcomes disagree. As it turns out, the correlations must be of the form 14

$$
\begin{aligned}
b=a \oplus 1 \text { or } a=b \oplus 1: p\left(1,0 \mid \mathcal{M}_{a}, \mathcal{M}_{b} ; \mathcal{P}\right) & =\frac{1}{2} \\
p\left(0,1 \mid \mathcal{M}_{a}, \mathcal{M}_{b} ; \mathcal{P}\right) & =\frac{1}{2} \\
b=a: p\left(0,0 \mid \mathcal{M}_{a}, \mathcal{M}_{b} ; \mathcal{P}\right) & =\frac{1}{2} \\
p\left(1,1 \mid \mathcal{M}_{a}, \mathcal{M}_{b} ; \mathcal{P}\right) & =\frac{1}{2} .
\end{aligned}
$$

We do not specify the nature of the correlation for other values of $a$ and $b$.

We can define the average probability of success as

$$
\begin{aligned}
R_{n} & \equiv \frac{1}{3 n} \sum_{a, b: b=a \oplus 1 \text { or } a=b \oplus 1} p\left(A_{a} \neq B_{b} \mid \mathcal{M}_{a}, \mathcal{M}_{b} ; \mathcal{P}\right) \\
& +\frac{1}{3 n} \sum_{a, b: b=a} p\left(A_{a}=B_{b} \mid \mathcal{M}_{a}, \mathcal{M}_{b} ; \mathcal{P}\right)
\end{aligned}
$$

It is evident that with a local strategy if one has perfect correlation when $a=b$, then when $a=b \oplus 1$ or $b=a \oplus$ 1 , one can have perfect anti-correlation with probability at most $(n-1) / n$. Furthermore, no local strategy can do any better than this. Consequently, given that the conditions $a=b, a=b \oplus 1$, and $b=a \oplus 1$ arise with probability $1 / 3$ each, the winning probability with a local strategy is upper bounded by 15

$$
R_{n} \leq R_{n}^{\text {local }}=\frac{1}{3}+\frac{2}{3} \frac{n-1}{n}=1-\frac{2}{3 n} .
$$

Quantum theory can violate this inequality. Using the same entangled state as above, we generalize Eq. (41) to

$$
\hat{A}_{a}=\cos \varphi_{a} \hat{\sigma}_{z}+\sin \varphi_{a} \hat{\sigma}_{x},
$$

where $\varphi_{a}=\frac{n-1}{n} \pi(a-1)$, and likewise for the $\hat{B}_{b}$ (Fig. (4). There are $3 n$ kinds of measurement statistics that appear in $R_{n}$. We consider each in turn. For the $n$ terms wherein $a=b$, we obtain perfect correlation with probability 1 , while for the $n$ terms wherein $a=b \oplus 1$ and the $n$ terms wherein $b=a \oplus 1$, we obtain anti-correlated outcomes

14 The proof of this proceeds analogously to the one given in Appendix $\mathrm{D}$ for the specific case of $n=3$.

15 It is worth noting that none of the following Bell inequalities is facet-inducing (following the terminology of Ref. [35]), or tight (following the terminology of Ref. [36, 37]). That is, they do not correspond to the boundary of the set of locally causal correlations with maximal dimension. with probability $\cos ^{2}(\pi / 2 n)$. In all then, we find the corresponding probability of success as:

$$
R_{n}^{\text {quantum }}=\frac{1}{3}+\frac{2}{3} \cos ^{2} \frac{\pi}{2 n} \simeq 1-\frac{\pi^{2}}{6 n^{2}} .
$$

Once again, for a large number of suitors, the seer can choose $n$, the number of measurement settings, to ensure that with very high probability all of the suitors will lose, despite their classically founded expectation that one of their number is very likely to win.
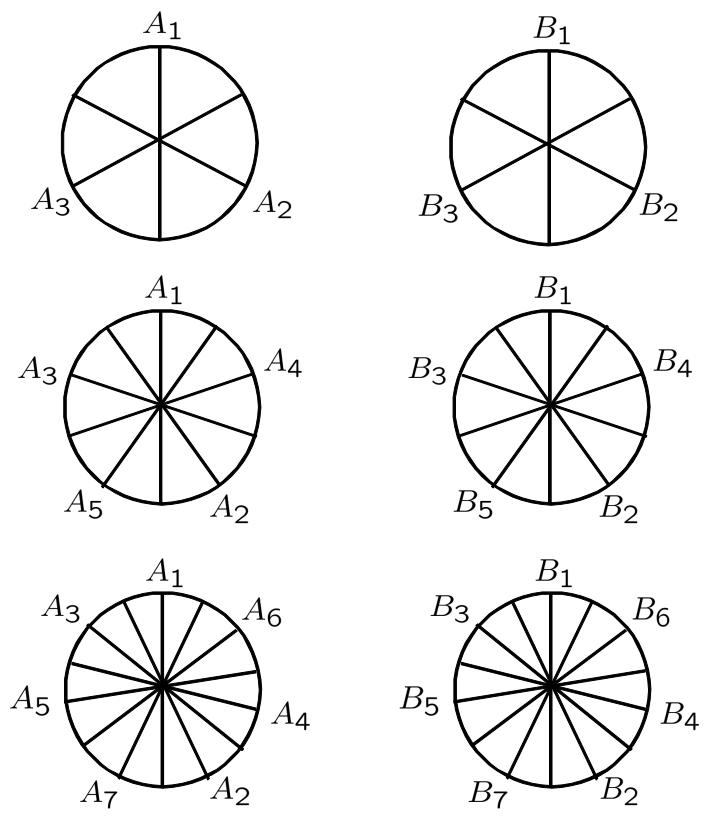

FIG. 4. Representation of the observables used in the Mermin-type proofs of nonlocality for $n=3,5$ and 7 . The observables are depicted by lines in a plane of the Bloch sphere. The vertices of each line correspond to the eigenvectors of the associated observable, with the labeled vertex associated with eigenvalue 1.

\section{Connection to previous work}

An analogous game is discussed by Vaidman 38] who considers a slightly different narrative device: a necklace having an even number $n$ of beads each of which can be one of two colors and such that one finds all adjacent beads to be of different colors except for the first and last beads which are of the same color. It is clear that by replacing the first and last beads by a single bead, we have precisely the correlations considered above. Another variation of the game was considered by Braunstein and Caves [39]: there, perfect correlation is required for all adjacent pairs of measurements except that between the first and the last, in which case perfect anti-correlation is required. This game gives rise to the so-called "chained Bell inequalities" 39]. 
The problem of maximizing the winning probability $R_{n}$ is also relevant to the strength of a two-prover interactive proof system of the type described by Cleve et al. 34]. The two provers are taken to be two agents of the seer (one sent to Abydos and the other to Babylon), while the suitor is the verifier. The provers' task is to convince the verifier that a cyclic graph with an odd number $n$ of vertices is 2-colorable (despite the fact that it is not). The verifier sends the name of a vertex to each prover such that the two vertices are either the same or adjacent. The provers, who cannot communicate with one another, must each respond with a color. The existence of systems generating the nonlocal OS correlations would provide the provers with a perfect winning strategy.

Cleve et al. have analyzed a two-player interactive proof, called the odd cycle game, which is very similar to the one we consider here. The odd cycle game is another natural generalization of Specker's parable to a pair of systems where for a given measurement on the Abydosian system, there are two rather than three options for the measurement on the Babylonian system: it is the same, i.e. $b=a$, or it has index one higher, i.e. $b=a \oplus 1$. The possibility of $a=b \oplus 1$, which is allowed in the game we have considered, and ensures symmetry between the two players, is excluded in the odd cycle game. 16

\section{From OS correlations to PR-box correlations}

Another way of generalizing the single-query 3-box OS correlations to a separated pair of parties is to imagine that each party has a 3-box system, but the first party only ever opens the first or second box, while the second party only ever opens the second or third box. If we imagine that there is correlation when they both open the second box and anti-correlation otherwise, then this set of measurements is already sufficient to obtain a contradiction with a local model, Specifically, the local deterministic values must satisfy

$$
\begin{aligned}
& \bar{A}_{2} \bar{B}_{2}=+1, \\
& \bar{A}_{1} \bar{B}_{2}=-1, \\
& \bar{A}_{1} \bar{B}_{3}=-1, \\
& \bar{A}_{2} \bar{B}_{3}=-1,
\end{aligned}
$$

16 The upper bound on the winning probability with a local strategy for the odd cycle game is clearly $R_{n}^{\text {local }} \leq \frac{1}{2}+\frac{1}{2} \frac{n-1}{n}=1-$ $\frac{1}{2 n}$. The maximal quantum violation, which is determined in Ref. [34], is achieved if the measurements on Alice's system are the spin operators $A_{a}$ in Eq. [45, while the measurements on Bob's system are a rotation by an angle of $\pi / 4 n$ of the spin operators $B_{b}$ in Eq. 45). In this case, for the $n$ terms wherein $a=b$, we have correlation with probability $\cos ^{2}(\pi / 4 n)$, and for the $n$ terms wherein $b=a \oplus 1$, we have anti-correlation with probability $\cos ^{2}(\pi / 4 n)$, such that $R_{n}^{\text {quantum }}=\cos ^{2}(\pi / 4 n) \simeq 1-$ $\frac{\pi^{2}}{16 n^{2}}$. but the product of the left-hand-sides is $\bar{A}_{1}^{2} \bar{A}_{2}^{2} \bar{B}_{1}^{2} \bar{B}_{2}^{2}=$ +1 , while the product of the right-hand-sides is -1 . The correlations of Eq. (47) are precisely the PR box correlations [17] that have been extensively studied in recent years.

\section{E. Hardy-type no-go theorems for Bell-local models}

In outcome-deterministic ontological models that are local or noncontextual, implications among value assignments of observables are transitive because these value assignments do not depend on the context (local or remote) of the measurement. The failure of the transitivity of implication therefore implies the impossibility of such models. Again, we find that this conclusion has been reached before in the literature on nonlocality. Specifically, the Hardy-type proof of nonlocality [11] can be expressed in this fashion [40], a fact that was first noted by Stapp [41] (for a simplified account, see Refs. 42, 43]).

We begin by presenting Hardy's proof of nonlocality in its standard form. It uses a pair of binary-outcome observables on each wing of the experiment. Hardy demonstrated a way of choosing these observables such that for any partially entangled pure state, the correlations between these observables satisfy:

$$
\begin{aligned}
& A_{1}=1 \Longrightarrow B_{1}=1, \\
& B_{2}=1 \Longrightarrow A_{2}=1,
\end{aligned}
$$

while

$$
\text { sometimes }\left(A_{1}=1 \text { and } B_{2}=1\right)
$$

(i.e. with probability $p_{\text {Hardy }} \equiv p\left(A_{1}=1\right.$ and $\left.B_{2}=1\right)>$ $0)$, and

$$
\text { never }\left(A_{2}=1 \text { and } B_{1}=1\right) \text {. }
$$

We can express this as a failure of the transitivity of implication as follows. From Eqs. (48), (51) and (49)) (in its contrapositive form), we infer respectively,

$$
\begin{aligned}
& A_{1}=1 \Longrightarrow B_{1}=1, \\
& B_{1}=1 \Longrightarrow A_{2}=0, \\
& A_{2}=0 \Longrightarrow B_{2}=0 .
\end{aligned}
$$

which we summarize graphically by

$$
\begin{gathered}
A_{1}=1 \Longrightarrow B_{1}=1 \\
A_{2}=0 \Longrightarrow B_{2}=0
\end{gathered}
$$

If transitivity held, then these three inferences would imply that

$$
A_{1}=1 \Longrightarrow B_{2}=0 \text {. }
$$


However, this contradicts Eq. (50) and consequently transitivity must fail. More explicitly, taking $\Longrightarrow$ to be material implication, the negation of Eq. (55) is the conjunction of $A_{1}=1$ and $B_{2}=1$,

$$
\neg\left(A_{1}=1 \Longrightarrow B_{2}=0\right)=\left(A_{1}=1 \text { and } B_{2}=1\right),
$$

so that the probability $p_{\text {Hardy }} \equiv p\left(A_{1}=1\right.$ and $\left.B_{2}=1\right)$ quantifies the frequency with which the transitivity of implication fails.

We now consider the status of this sort of proof for the PR box. By relabeling the outcomes of the standard PR box, one can obtain correlations of the form

$$
\begin{aligned}
& A_{1}=B_{1} \\
& A_{1}=B_{2} \\
& A_{2}=B_{1} \oplus 1 \\
& A_{2}=B_{2},
\end{aligned}
$$

with marginals of the form $p\left(A_{1}=0\right)=p\left(A_{2}=0\right)=$ $p\left(B_{1}=0\right)=p\left(B_{2}=0\right)=1 / 2$. Eqs. (57), (59) and (60) imply the inferences of Eqs. (52), (53), and (54) respectively. Meanwhile, Eq. (58), together with the fact that $p\left(A_{1}=1\right)=1 / 2$, implies that sometimes $A_{1}=1$ and $B_{2}=1$, or equivalently, that sometimes Eq. (55) fails, so that we have a contradiction with transitivity. Indeed, the probability of this occurring is $p_{\text {Hardy }}=p\left(A_{1}=1\right.$ and $\left.B_{2}=1\right)=1 / 2$.

Actually, $p_{\text {Hardy }}$ only quantifies the probability for one particular kind of contradiction, which requires $A_{1}=1$ to get going. In the rest of the cases, where $A_{1}=0$, we still obtain a contradiction because Eqs. (57), (59) and (60) also imply inferences of the form of Eqs. (52), (53), and (54) where $A_{a} \Leftrightarrow A_{a} \oplus 1$ and $B_{b} \Leftrightarrow B_{b} \oplus 1$. Transitivity then implies that $A_{1}=0 \Longrightarrow B_{2}=1$, while Eq. (58) contradicts this. So one obtains a contradiction with certainty for the PR box.

There is another aspect of these PR box implications that cannot be emulated by quantum theory which has recently been pointed out by Fritz [44]: if one supplements the implications in Eqs. (52)-(554) with the implication $B_{2}=1 \Longrightarrow A_{1}=1$ or any of the two reverse implications, that is, $A_{2}=0 \Longrightarrow B_{1}=1$, or $B_{2}=0 \Longrightarrow A_{2}=0$, then the resulting set of constraints cannot be satisfied by any quantum state and set of projective measurements.

As discussed in the introduction, and rehearsed in Sec. IIIC Specker introduced his parable of the overprotective seer in order to demonstrate the possibility of a logic wherein there is a failure of the transitivity of implication. One therefore expects that the nonlocal OS correlations from Sec. IVA which are based on Specker's parable, ought to provide a proof of nonlocality via such a failure of transitivity. This is indeed the case, as we now show. The nonlocal OS correlations, $c f$. Eq. (31), imply the following chain of implications

$$
\begin{gathered}
A_{1}=1 \Longrightarrow B_{1}=1 \\
A_{2}=0 \Longrightarrow B_{2}=0 \\
\qquad \\
A_{3}=1 \Longrightarrow B_{3}=1
\end{gathered}
$$

If the transitivity of implication held, we would have

$$
A_{1}=1 \Longrightarrow B_{3}=1 \text {. }
$$

However, Eq. (31) together with the fact that $p\left(A_{1}=\right.$ 1) $=1 / 2, c f$. Eq. (35), implies that sometimes $A_{1}=1$ and $B_{3}=0$, which contradicts Eq. (61). Indeed, we achieve this contradiction with probability $p_{\text {Hardy }}=$ $p\left(A_{1}=0\right.$ and $\left.B_{3}=1\right)=1 / 2$. As with the PR box, one can obtain a contradiction with certainty also in the cases where $A_{1}=0$.

Although the nonlocal OS correlations cannot be achieved in quantum theory, it is interesting to ask whether the particular contradiction constructed above might be achieved with some nonzero probability for some choice of state and observables. Indeed, this is possible. In particular, this can be achieved with $p^{\text {Hardy }}=144 /(27+\sqrt{3})^{2} \approx 0.17443$ by using the quantum state $|\psi\rangle=\left(1+\eta^{2}\right)^{-1 / 2}(|0\rangle|0\rangle-\eta|1\rangle|1\rangle)$ and the projectors defined by:

$$
\hat{A}_{a}=\left|\uparrow_{a}^{(A)}\right\rangle\left\langle\uparrow_{a}^{(A)}\left|, \quad \hat{B}_{b}=\right| \uparrow_{b}^{(B)}\right\rangle\left\langle\uparrow_{b}^{(B)}\right|,
$$

where

$$
\begin{gathered}
\left|\uparrow_{a}^{(A)}\right\rangle=\left\{\begin{array}{l}
\frac{1}{\sqrt{1+\kappa_{1}^{2}}}\left(\kappa_{1}|0\rangle+|1\rangle\right): a=1, \\
\frac{1}{\sqrt{1+\kappa_{2}^{2}}}\left(|0\rangle-\kappa_{2}|1\rangle\right): a=2, \\
\frac{1}{\sqrt{1+\kappa_{3}^{2}}}\left(|0\rangle+\kappa_{3}|1\rangle\right): a=3,
\end{array}\right. \\
\left|\uparrow_{b}^{(B)}\right\rangle=\left\{\begin{array}{l}
\frac{1}{\sqrt{1+\kappa_{3}^{2}}}\left(-\kappa_{3}|0\rangle+|1\rangle\right): b=1, \\
\frac{1}{\sqrt{1+\kappa_{2}^{2}}}\left(\kappa_{2}|0\rangle+|1\rangle\right): b=2, \\
\frac{1}{\sqrt{1+\kappa_{1}^{2}}}\left(|0\rangle-\kappa_{1}|1\rangle\right): b=3,
\end{array}\right.
\end{gathered}
$$

$\kappa_{a}=\eta^{(a+1 \bmod 3)+\frac{1}{2}}$, and $\eta=\sqrt{3}$.

The above Hardy-type proof of nonlocality via the failure of the transitivity of implications is entirely equivalent to the proof of nonlocality due to Boschi et al. [40]. Note that a slightly stronger contradiction with $p^{\text {Hardy }} \approx$ 0.17455 can be obtained with a different choice of $\eta$ [40]. Moreover, this latter value of $p^{\text {Hardy }}$ is only marginally different from the quantum-mechanical upper bound $p^{\text {Hardy }} \leq 0.17456$ obtained from the tools of Ref. [23]. This suggests that the strongest contradiction in this scenario may already be achievable using a two-qubit partially entangled pure state 17

17 See, however, Ref. 45] for some strong evidence that in some 
It is also worth noting that by considering a similar setup that involves an increasing number of boxes, and hence a longer chain of intransitive implications, quantum theory actually provides a contradiction with increasing $p^{\text {Hardy }}$ that asymptotes to $50 \%$ [40].

We end this section with a demonstration that there is a particular kind of failure of transitivity that one does not find in quantum theory. We begin by noting that with a PR box, we can get a contradiction with the transitivity of implication in a manner which is different from that of Hardy's proof, and in some ways more striking. In addition to deriving Eqs. (52), (53) and (54) from Eqs. (57), (59) and (60), we can derive

$$
B_{2}=0 \Longrightarrow A_{1}=0 \text {. }
$$

Graphically, the chain of inferences is

$$
\begin{aligned}
& A_{1}=1 \Longrightarrow B_{1}=1 \\
& A_{2}=0 \Longrightarrow B_{2}=0 \\
& A_{1}=0
\end{aligned}
$$

Were transitivity of implication to hold, we would conclude that $A_{1}=1 \Longrightarrow A_{1}=0$, which, together with the fact that $p\left(A_{1}=1\right)=1 / 2$, yields a contradiction. This sort of proof is also available for the nonlocal OS correlations. It can be characterized as providing a sequence of inferences about values of observables wherein the consequent of the last inference contradicts the antecedent of the first inference. The question is whether this sort of contradiction can be achieved in quantum theory. As it turns out, it cannot, as we now demonstrate.

Consider an arbitrary pure bi-partite state $|\Psi\rangle \in \mathcal{H}^{A} \otimes$ $\mathcal{H}^{B}$ where $\mathcal{H}^{A}$ and $\mathcal{H}^{B}$ are Hilbert spaces of dimension d. Defining $\{|k\rangle\}_{k=1}^{d}$ to be an orthonormal basis of $\mathcal{H}$, $\rho$ to be a density operator, $\hat{\mathbf{1}}$ to be the identity operator and $U$ to be a unitary operator, we can always write $|\Psi\rangle$ in the form

$$
|\Psi\rangle=(\hat{\mathbf{1}} \otimes U \sqrt{\rho}) \sum_{k}|k\rangle \otimes|k\rangle .
$$

Now suppose that one measures system $A$ with the $\operatorname{POVM}\{|\phi\rangle\langle\phi|, \hat{\mathbf{1}}-| \phi\rangle\langle\phi|\}$ and one obtains the $|\phi\rangle\langle\phi|$ outcome. This leads to an updating of the description of the state of system $B$ to

$$
|\chi\rangle \equiv \mathcal{N}_{\chi} U \sqrt{\rho}\left|\phi^{*}\right\rangle
$$

cases, it may require infinite-dimensional Hilbert space to achieve the strongest correlations allowed by quantum mechanics even though the two-qubit correlations are only marginally different from the quantum mechanical upper bound derived from the tools of Ref. [23]. where

$$
\left|\phi^{*}\right\rangle \equiv \sum_{k}\langle k \mid \phi\rangle^{*}|k\rangle,
$$

and $\mathcal{N}_{\chi}$ is a normalization factor. Consequently, a subsequent measurement on system $B$ of the POVM $\{|\chi\rangle\langle\chi|, \hat{\mathbf{1}}-| \chi\rangle\langle\chi|\}$ will yield the $|\chi\rangle\langle\chi|$ outcome with certainty.

Next, consider the experiment wherein $\{|\phi\rangle\langle\phi|, \hat{\mathbf{1}}-| \phi\rangle\langle\phi|\}$ is not made on $A$, but the measurement $\{|\chi\rangle\langle\chi|, \hat{\mathbf{1}}-\{|\chi\rangle\langle\chi|\}$ is made on $B$ and the outcome $|\chi\rangle\langle\chi|$ is obtained. One then updates the description of the state of system $A$ to

$$
\begin{aligned}
\left|\phi^{\prime}\right\rangle & \propto\left\langle\chi\left|(\hat{\mathbf{1}} \otimes U \sqrt{\rho}) \sum_{k}\right| k\right\rangle \otimes|k\rangle \\
& \propto\left\langle\phi^{*}\left|(\hat{\mathbf{1}} \otimes \rho) \sum_{k}\right| k\right\rangle \otimes|k\rangle \\
& \propto\left\langle\phi^{*}\left|\left(\rho^{T} \otimes \hat{\mathbf{1}}\right) \sum_{k}\right| k\right\rangle \otimes|k\rangle \\
\left|\phi^{\prime}\right\rangle & =\mathcal{N}_{\phi^{\prime}} \rho^{T}|\phi\rangle,
\end{aligned}
$$

where $\mathcal{N}_{\phi^{\prime}}$ is a normalization factor. A subsequent measurement on system $A$ of the POVM $\left\{\left|\phi^{\prime}\right\rangle\left\langle\phi^{\prime}|, \hat{\mathbf{1}}-| \phi^{\prime}\right\rangle\left\langle\phi^{\prime}\right|\right\}$ will then yield the $\left|\phi^{\prime}\right\rangle\left\langle\phi^{\prime}\right|$ outcome with certainty.

The state $|\chi\rangle$ on $B$ is called the relative state to $|\phi\rangle$ on $A$ given $|\Psi\rangle$ on $A B$ [46]. Similarly, $\left|\phi^{\prime}\right\rangle$ on $A$ is the relative state to $|\chi\rangle$ on $B$ given $|\Psi\rangle$ on $A B$. If we find a particular state on one system, then we are certain to find the relative state on the other should we measure for it. Consequently, we can consider an arbitrary chain of such pairs of measurements, and at every step in the chain we can make a perfect inference from the positive outcome of one to the positive outcome of the other.

We pause at this point in the proof to note that this analysis provides a particularly simple way of understanding Hardy's proof of nonlocality. Using reasoning analogous to that above, the relative state to $\left|\phi^{\prime}\right\rangle$ is $\left|\chi^{\prime}\right\rangle$ where $\left|\chi^{\prime}\right\rangle \equiv U \rho U^{\dagger}|\chi\rangle$ (note that there clearly exist choices of $\rho$ and $U$ such that $\left.\left|\chi^{\prime}\right\rangle \neq|\chi\rangle\right)$. If the transitivity of implication held, then by this sequence of perfect inferences, we would conclude that whenever $|\phi\rangle\langle\phi|$ is found on $A$, it would be the case that $\left|\chi^{\prime}\right\rangle\left\langle\chi^{\prime}\right|$ is necessarily found on $B$. However, this conclusion is false because the relative state to $|\phi\rangle$ is $|\chi\rangle$, so that the probability of finding $\left|\chi^{\prime}\right\rangle\left\langle\chi^{\prime}\right|$ on $B$ is $\left|\left\langle\chi^{\prime} \mid \chi\right\rangle\right|^{2}$ which is less than one if $\left|\chi^{\prime}\right\rangle \neq|\chi\rangle$. Thus transitivity must fail.

We now show that a quantum proof of nonlocality cannot be constructed in terms of a sequence of inferences wherein the consequent of the last inference contradicts the antecedent of the first. We define a set of $N$ observables on $A$, each of which is projective, namely, $\left\{\left|\phi^{(i)}\right\rangle\left\langle\phi^{(i)}|| i=1, \ldots, N\right\}\right.$ and a set of $N$ similar observables on $B,\left\{\left|\chi^{(i)}\right\rangle\left\langle\chi^{(i)}|| i=1, \ldots, N\right\}\right.$. Here, $\left|\chi^{(i)}\right\rangle$ is the relative state to $\left|\phi^{(i)}\right\rangle$ and $\left|\phi^{(i+1)}\right\rangle$ is the relative 
state to $\left|\chi^{(i)}\right\rangle$. This implies that we can infer from finding $\left|\phi^{(i)}\right\rangle\left\langle\phi^{(i)}\right|$ on $A$ to the necessity of finding $\left|\chi^{(i)}\right\rangle\left\langle\chi^{(i)}\right|$ on $B$, and from finding $\left|\chi^{(i)}\right\rangle\left\langle\chi^{(i)}\right|$ on $B$ to the necessity of finding $\left|\phi^{(i+1)}\right\rangle\left\langle\phi^{(i+1)}\right|$ on $A$. If transitivity of implication held, then we could chain these inferences together such that from finding $\left|\phi^{(1)}\right\rangle\left\langle\phi^{(1)}\right|$ on $A$, we would infer the necessity of finding $\left|\phi^{(N)}\right\rangle\left\langle\phi^{(N)}\right|$ on $A$.

The question is whether we can ever have such a chain where $\left|\phi^{(N)}\right\rangle$ is orthogonal to $\left|\phi^{(1)}\right\rangle$. Note from the analysis above that $\left|\phi^{(i+1)}\right\rangle \propto \rho^{T}\left|\phi^{(i)}\right\rangle$, so that

$$
\left|\phi^{(N)}\right\rangle \propto\left(\rho^{T}\right)^{N}\left|\phi^{(1)}\right\rangle \text {. }
$$

Therefore the condition for orthogonality is

$$
\left|\left\langle\phi^{(1)}\left|\left(\rho^{T}\right)^{N}\right| \phi^{(1)}\right\rangle\right|^{2}=0
$$

Given the non-negativity of $\rho$ (and hence of $\rho^{T}$ and $\left.\left(\rho^{T}\right)^{N}\right)$, this condition is only satisfied if $\rho^{T}\left|\phi^{(1)}\right\rangle=0$, but this would imply that the probability of finding $\left|\phi^{(1)}\right\rangle\left\langle\phi^{(1)}\right|$ on the bipartite state $|\Psi\rangle$ vanishes. In other words, the only bipartite state for which we can have a chain of inference wherein the final consequent denies the initial antecedent is one that denies the initial antecedent. Therefore, such a contradiction cannot be achieved. This is in contrast to what occurs in the case of $\mathrm{PR}$ boxes and the nonlocal OS correlations, and is therefore a feature which distinguishes quantum theory from these foil theories. It is interesting to note, however, that in quantum proofs of contextuality one can find a chain of inferences where the final consequent denies the initial antecedent and the initial antecedent is sometimes true, as shown in Sec. IIIC,

\section{FRUSTRATED NETWORKS}

It is instructive to consider a network representation of the various correlations that we have considered thus far. The bit associated with the outcome of a binary-outcome measurement (this is the only type of measurement we've considered) is associated with a node. Perfect positive correlation between outcomes of distinct measurements is represented by a solid line between the nodes, perfect negative correlation by a dashed line. Such representations of correlations have been discussed before in the context of nonlocality proofs, in particular by Mitchell, Popescu and Roberts [47] and in the Ph.D. thesis of Collins [48] and by Schmidt [49].

Fig. 5 provides network representations of the extremal correlations that were used in the no-go theorems for measurement-noncontextual outcome-deterministic models. The triangular network represents the OS correlations in Specker's parable; the square network represents the PR-box correlations (understood as a proof of contextuality, i.e. where the four measurements are considered to be implemented in one spatial location); the pentagonal network represents the extremal version of the correlations in Klyachko's no-go theorem; the hexagonal network represents the kind of correlations described by Vaidman in Ref. [38].
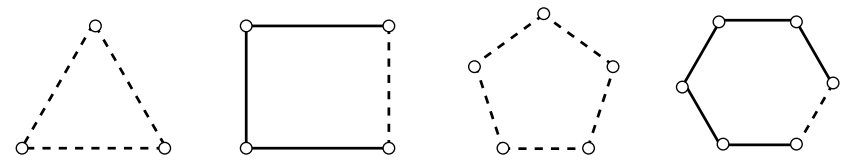

FIG. 5. Frustrated networks representing the extremal correlations in various proofs of contextuality.

Fig. 6]provides network representations of the extremal correlations that were used in proofs of nonlocality. We have labeled the nodes to highlight the spatial region in which each of the outcomes occurs. The network on the left, which is graph-isomorphic to the square network above, represents the correlations generated by a PR-box [17]. The network on the right depicts the correlations found in the separated pair of single-query 3-box systems of Sec. IV]
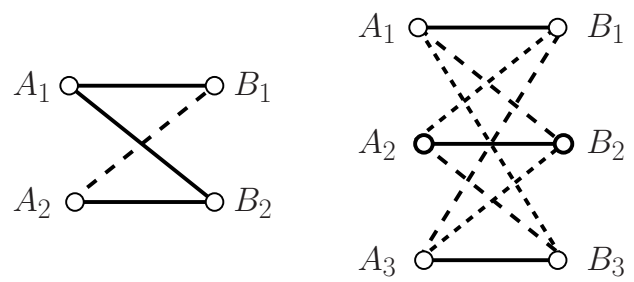

FIG. 6. Frustrated networks representing the extremal correlations in various proofs of nonlocality.

Let the bit describing whether there is an even or an odd number of dashed lines along a path be called the parity of the path. We shall say that a network is frustrated if for any pair of nodes, there exist paths with different parities connecting those nodes. Clearly, each of the networks in Fig. 5 is frustrated. It is this frustration which captures the impossibility of an outcomedeterministic measurement-noncontextual model of these correlations. For the networks given in Fig. 6, this impossibility also gives rise to a simple proof of nonlocality of the depicted correlations.

For any network, we can determine whether or not it is frustrated by looking only at its cycles. This is because frustration occurs when there are two paths with differing parities and this fact will reveal itself by examining the cycle consisting of that pair of paths. Thus, to see the ways in which a network can be frustrated, it suffices to consider the ways in which cycles can be frustrated. For any integer number of nodes, it is straightforward to find all the frustrated cycles with that number of nodes. For two nodes, there is only a single path and therefore no possibility for frustration. At 3 nodes, the frustrated networks are those indicated in Fig. 7. The case of two 
correlations and one anti-correlation corresponds, in the imagery of Specker's parable, to a case where if boxes 1 and 2 or boxes 1 and 3 are opened, one finds the same outcome, but if boxes 2 and 3 are opened, the outcomes always differ. Note, however, that these different networks are equivalent up to a relabeling of the outcomes and consequently represent essentially the same correlations.
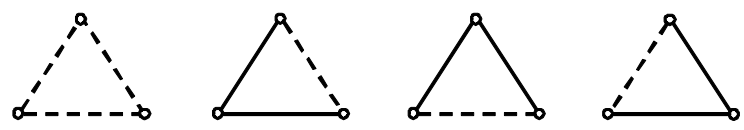

FIG. 7. All the ways in which a triangular network can be frustrated.

Indeed, all the frustrated networks with a given number of nodes can be obtained one from another by a relabeling of the outcomes. It therefore suffices to consider a single representative of the equivalence class of frustrated networks with a given number of nodes.

It is also possible to have a similar graphical representation for some of the no-go theorems for noncontextuality and locality that are based on a failure of transitivity of implication. We represent a set of implications among the values of binary-outcome observables by a directed graph with decorated edges. The implications of interest are of the form: $X_{1}=x \Longrightarrow X_{2}=y$ where $x, y \in\{0,1\}$ and either $y=x$ or $y=x \oplus 1$. We depict this by inserting a directed edge (i.e. an arrow) from the node for $X_{1}$ to the node for $X_{2}$ and decorating the base of the arrow with the value $x$; the directed edge is solid if $y=x$ and dashed if $y=x \oplus 1$. Note that this implication can also be written in its contrapositive form as $X_{2}=y \oplus 1 \Longrightarrow X_{1}=x \oplus 1$. Therefore, we can always represent the same implication with an arrow in the opposite direction. When reversing an arrow, the value decorating the arrow stays the same if the arrow is solid and flips if the arrow is dashed.

If the parity is odd around a closed loop in such a directed graph, then the antecedent of the first implication is denied by the consequent of the last implication. Therefore, as long as the antecedent has non-zero probability, we have a failure of the transitivity of implication. Such a directed network is said to be frustrated.

In the introduction, we described how Specker's parable implies a failure of the transitivity of implication (under the assumption that value-assignments to observables are context-independent). Letting $s_{i}$ denote the proposition that $X_{i}=1$ (box $i$ contains a gem), the set of implications are: $s_{1} \Longrightarrow \neg s_{2}, \neg s_{2} \Longrightarrow s_{3}$, and $s_{3} \Longrightarrow \neg s_{1}$. These are represented by the directed network of Fig. 8(a), which is clearly frustrated. The set of implications that are used in the transitivity-based no-go theorem of Sec. IIIC are represented by the pentagonal version of this directed network, Fig. 8(b), which is also frustrated.

Unlike the undirected frustrated networks, which are composed of a set of correlations some or all of which (a)

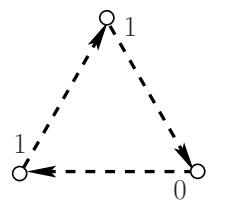

(b)

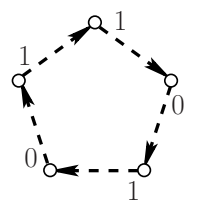

FIG. 8. Some frustrated directed networks corresponding to contextual correlations.

are only approximated by the quantum correlations, the directed frustrated network of Fig. 8(b) is an exact specification of implications one finds in quantum theory, specifically, those described in the proof of Sec. IIC. The only sense in which one could imagine a theory being "more contextual", according to this sort of proof, is by assigning a higher probability to the contradictiongenerating valuation of the first observable in the chain. An extremal version of such a proof would be one wherein both possible valuations of the first observable yielded a contradiction. We conjecture that such a proof cannot be found in quantum theory.

Finally, the "striking" form of the PR box correlations, presented in Sec. IVE and associated with the set of implications below Eq. (62), is represented by the frustrated directed network in Fig. 9(a), and the generalization of this to the case of the nonlocal OS correlations is represented in Fig. 9(b). As was shown at the end
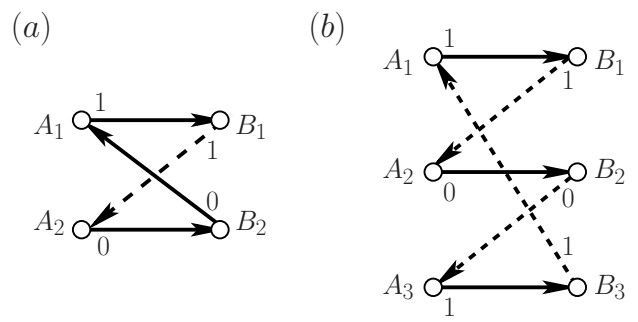

FIG. 9. Some frustrated directed networks corresponding to Bell-nonlocal correlations.

of Sec. IVE it is not possible to find a quantum state and a set of observables that instantiates such a set of implications while assigning a nonzero probability to the contradiction-generating valuation of the first observable.

\section{NO-GO THEOREMS FOR PREPARATION-NONCONTEXTUAL MODELS}

So far, in all of our quantum analogues of Specker's parable, the correlations examined were between the outcomes of pairs of measurements that could be implemented jointly. In this section, we consider the possibility of achieving these correlations between the outcomes of pairs of measurements that are implemented consecu- 
tively 18

It is important to recognize that one need not rule out the possibility of consecutive measurements to ensure the impossibility of joint measurements. The original version of the Specker parable is misleading in this respect. It asks us to imagine that after opening two boxes, one is simply unable to open the third (as if its lid were glued shut with an unbreakable seal). The literal generalization to arbitrary measurements $\mathcal{M}_{1}, \mathcal{M}_{2}$ and $\mathcal{M}_{3}$ that can be measured jointly pairwise but not triplewise would seem to be that if $\mathcal{M}_{1}$ and $\mathcal{M}_{2}$ have been implemented, a mysterious force prevents us from carrying out the instructions that correspond to implementing $\mathcal{M}_{3}$. However, this conclusion does not follow from a denial of joint measurability as it is defined in Sec. IIA. One can always implement $\mathcal{M}_{3}$ following a measurement of $\mathcal{M}_{1}$ and $\mathcal{M}_{2}$ on a preparation $\mathcal{P}$. It is just that the statistics of outcomes of $\mathcal{M}_{3}$ that one thereby obtains is not the same as one would have obtained if $\mathcal{M}_{3}$ were implemented on $\mathcal{P}$ directly. To be precise, if the joint statistics of outcomes of a pair of measurements $\mathcal{M}$ and $\mathcal{M}^{\prime}$ are independent of the order in which they are implemented, then the consecutive implementation of the two measurements constitutes a joint measurement of $\mathcal{M}$ and $\mathcal{M}^{\prime}$ by the definition of Sec. IIA. Consequently, a denial of joint measurability implies a denial of the invariance of statistics under a reordering of the measurements. This way of interpreting a lack of joint measurability is precisely the one that is familiar from the quantum theory of projective measurements.

To see how the OS correlations might obtain for consecutive measurements, we present a new parable. We consider a single-query 3-box system, that is, one where only a single box can be opened at a time. A pair of boxes can be opened consecutively, but the second boxopening need not reproduce the statistics of outcomes that would have been observed had it been opened first. In this sense, the measurements associated with opening distinct boxes cannot be implemented jointly.

We now get to the specifics of the correlations, which are inspired by the original Specker parable. We assume that there is a special preparation $\mathcal{P}_{*}$ of the 3 -box system, such that if the same box is opened at the two times, then the same outcome is found, while if different boxes are opened at the two times, then different outcomes are found.

So far, there is nothing in this set of correlations that prohibits their being explained by a generalizednoncontextual ontological model. Because no two measurements are ever implemented jointly in this parable, there is no sense in which any measurement has a nontrivial context upon which its ontological representation

18 Because implementing the first measurement and selecting a particular outcome constitutes a preparation, one can equally well describe this section as a consideration of the possibility of achieving analogues of the OS correlations between preparations and measurements. This is discussed further below. might depend. Indeed, there are ontological models that explain the correlations easily. They need only posit that the first measurement disturbs the ontic state of the three-box system in order to enforce the appropriate correlations. For instance, suppose that three bits specify the gem occupation numbers of the three boxes and completely characterize the ontic state. It could be that finding a 0 (1) for a box forces the other two boxes to have occupation number 1(0). (Indeed, if the suitor is opening boxes on a table, this kind of disturbance to the ontic state might be enforced by having a hidden mechanism under the table that automatically inserts or removes gems from the two boxes that were not opened.)

To obtain a set of correlations that can challenge the assumption of generalized-noncontextuality, we need to modify the thought experiment slightly by adding the following assumption: in addition to the correlations described, it is the case that after the early measurement is complete, for every possible subsequent measurement (the theory may well allow more than the three measurements that are used in the protocol), it is impossible to obtain any information about the identity of the early measurement. We call this the trit-obliviousness condition (this terminology has its precedent in Ref. [50]).

Note that implementing the early measurement procedure and selecting a particular outcome constitutes a preparation. For each of the three possible measurement procedures, $M_{1}, M_{2}$ and $M_{3}$, and each of the outcomes 0 and 1 , we obtain a distinct preparation procedure. We denote these by $P_{1,0}, P_{1,1}, P_{2,0}, P_{2,1}, P_{3,0}$ and $P_{3,1}$ in an obvious notation. 19 We can also define the preparations that result when one chooses not to condition on the outcome of the measurement procedure. We denote these by $P_{1}, P_{2}$ and $P_{3}$. Finally, we denote the probability of obtaining outcome 0 when the first measurement $M_{t}$ is implemented on the special preparation $\mathcal{P}_{*}$ by $w_{t, 0} \equiv p\left(0 \mid M_{t} ; \mathcal{P}_{*}\right)$, and we define $w_{t, 1} \equiv 1-w_{t, 0}$. The statistics for the unconditional preparations are then given by

$$
p\left(X \mid M ; P_{t}\right)=w_{t, 0} p\left(X \mid M ; P_{t, 0}\right)+w_{t, 1} p\left(X \mid M ; P_{t, 1}\right) .
$$

The trit-obliviousness condition states that preparation procedures $P_{1}, P_{2}$ and $P_{3}$ are operationally equivalent, that is,

$$
\forall M: p\left(X \mid M ; P_{1}\right)=p\left(X \mid M ; P_{2}\right)=p\left(X \mid M ; P_{3}\right)
$$

We now see how the new parable might have troubles with generalized-noncontextuality. In Sec. IID, we defined a preparation-noncontextual ontological model

19 Note that measurement procedures $M_{1}$ and $M_{1}^{\prime}$ that are in the same operational equivalence class $\mathcal{M}_{1}$, may nonetheless define preparation procedures that fail to be operationally equivalent, because operational equivalence for preparation procedures is decided by the statistics of all possible subsequent measurements. 
to be one wherein operational equivalence of preparation procedures implies that they are represented by the same distributions in the ontological model, cf. Eq. (17). Thus, from Eq. (70) and preparation noncontextuality, we infer that

$$
p\left(\lambda \mid P_{1}\right)=p\left(\lambda \mid P_{2}\right)=p\left(\lambda \mid P_{3}\right) .
$$

Given that convex combinations of preparation procedures are represented in an ontological model by convex combinations of the associated distributions (see Ref. [12]), we infer from Eq. (71) that

$$
\begin{aligned}
& w_{1,0} p\left(\lambda \mid P_{1,0}\right)+w_{1,1} p\left(\lambda \mid P_{1,1}\right) \\
= & w_{2,0} p\left(\lambda \mid P_{2,0}\right)+w_{2,1} p\left(\lambda \mid P_{2,1}\right) \\
= & w_{3,0} p\left(\lambda \mid P_{3,0}\right)+w_{3,1} p\left(\lambda \mid P_{3,1}\right) .
\end{aligned}
$$

The "preparation context" is the specification of which of the three mixtures of preparation procedures was implemented, and the assumption of preparation noncontextuality is that the distribution over $\lambda$ does not depend on this context.

Equation (72) is a nontrivial constraint which is not necessarily consistent with the posited correlations between the preparation procedures $P_{1,0}, P_{1,1}, P_{2,0}, P_{2,1}$, $P_{3,0}$ and $P_{3,1}$ and the outcomes of the subsequent measurements of $M_{1}, M_{2}$ or $M_{3}$. Indeed, in the ontological model we proposed above, where the ontic state is a triple of bits specifying the occupation numbers of each box, the distributions corresponding to the six preparation procedures are:

$$
\begin{aligned}
& p\left(\lambda \mid P_{1,0}\right)=\delta_{\lambda,(0,1,1)} \\
& p\left(\lambda \mid P_{1,1}\right)=\delta_{\lambda,(1,0,0)} \\
& p\left(\lambda \mid P_{2,0}\right)=\delta_{\lambda,(1,0,1)} \\
& p\left(\lambda \mid P_{2,1}\right)=\delta_{\lambda,(0,1,0)} \\
& p\left(\lambda \mid P_{3,0}\right)=\delta_{\lambda,(1,1,0)} \\
& p\left(\lambda \mid P_{3,1}\right)=\delta_{\lambda,(0,0,1)},
\end{aligned}
$$

where $\delta$ denotes the Kronecker delta function. Supposing that $w_{1,0}=w_{2,0}=w_{3,0}=1 / 2$, we have

$$
\begin{aligned}
& p\left(\lambda \mid P_{1}\right)=\frac{1}{2} \delta_{\lambda,(0,1,1)}+\frac{1}{2} \delta_{\lambda,(1,0,0)}, \\
& p\left(\lambda \mid P_{2}\right)=\frac{1}{2} \delta_{\lambda,(1,0,1)}+\frac{1}{2} \delta_{\lambda,(0,1,0)}, \\
& p\left(\lambda \mid P_{3}\right)=\frac{1}{2} \delta_{\lambda,(1,1,0)}+\frac{1}{2} \delta_{\lambda,(0,0,1)},
\end{aligned}
$$

and therefore

$$
p\left(\lambda \mid P_{1}\right) \neq p\left(\lambda \mid P_{2}\right) \neq p\left(\lambda \mid P_{3}\right) .
$$

So we find that the distributions representing $P_{1}, P_{2}$ and $P_{3}$ in the ontological model are distinct even though these preparation procedures are operationally equivalent 20

\footnotetext{
${ }^{20}$ Remember that in the ontological model we are considering,
}

a violation of the assumption of preparation noncontextuality.

We have demonstrated that the simple ontological model suggested earlier to explain the two-time OS correlations cannot also satisfy the condition of tritobliviousness while preserving preparation noncontextuality. In the next subsection, we will show that no ontological model that can explain the OS correlations and the trit-oblivious condition can be preparationnoncontextual. In this sense, a suitor who is committed to generalized noncontextuality should be surprised if he sees the specified two-time correlations after having confirmed the trit-obliviousness condition.

It is useful to summarize the correlations that we have described above.

\section{A. Diachronic pair of single-query 3-box OS correlations}

There are six possible preparation procedures, denoted $P_{t, b}$ where $t \in\{1,2,3\}$ ( $t$ for trit) and $b \in\{0,1\}$, and three possible measurement procedures, denoted $M_{y}$ where $y \in\{1,2,3\}$. For simplicity, we assume that the prior over each of $t, b$ and $y$ to be uniform. The outcome $X$ of the measurement procedure $M_{y}$ given a preparation procedure $P_{t, b}$ is the following function of $t, b$ and $y$,

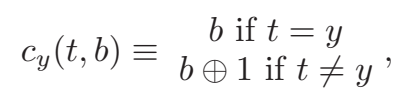

that is, the correlations are such that

$$
p\left(X=c_{y}(t, b) \mid M_{y} ; P_{t, b}\right)=1 .
$$

Finally, defining the effective preparation procedure $P_{t}$ as the mixture of $P_{t, 0}$ and $P_{t, 1}$, it is assumed that no measurement can reveal any information about which of $P_{1}, P_{2}$ or $P_{3}$ was implemented,

$$
\forall M: p\left(X \mid M ; P_{1}\right)=p\left(X \mid M ; P_{2}\right)=p\left(X \mid M ; P_{3}\right) .
$$

This is the trit-obliviousness condition.

Defining the average probability of success as

$$
R_{3} \equiv \frac{1}{18} \sum_{t, b, y} p\left(X=c_{y}(t, b) \mid M_{y} ; P_{t, b}\right)
$$

we can also characterize the two-time OS correlations as those achieving $R_{3}=1$. Using the trit-obliviousness condition, we shall see that the assumption of preparation

the measurement $M_{y}$ simply reveals the value of the $y$ th bit, that is, $p\left(X=\lambda_{y} \mid M_{y} ;\left(\lambda_{1}, \lambda_{2}, \lambda_{3}\right)\right)=1$. It follows that $\sum_{\lambda}$ $p\left(X=0 \mid M_{y} ; \lambda\right) p\left(\lambda \mid P_{t}\right)=\frac{1}{2}$ for all $y, t \in\{1,2,3\}$, and consequently, the two outcomes of $M_{y}$ occur with equal probability given a preparation procedure $P_{t}$. Therefore, the ontological model captures the fact that $P_{1}, P_{2}$ and $P_{3}$ are operationally indistinguishable. 
noncontextuality places a bound on the average probability of success, namely,

$$
R_{3} \leq R_{3}^{\mathrm{PNC}}=\frac{7}{9}
$$

We refer to this bound as a noncontextuality inequality.

The proof is as follows. For any measurement $M$, the probability of outcome $X$ given preparation $P_{t}$ is simply

$$
p\left(X \mid M ; P_{t}\right)=\frac{1}{2} \sum_{b \in\{0,1\}} p\left(X \mid M ; P_{t, b}\right) .
$$

Similarly, the probability of the ontic state $\lambda$ given an implementation of $P_{t}$ is simply

$$
p\left(\lambda \mid P_{t}\right)=\frac{1}{2} \sum_{b \in\{0,1\}} p\left(\lambda \mid P_{t, b}\right) .
$$

Given the trit-obliviousness condition, Eq. (85), and the assumption of preparation noncontextuality, Eq. (17), we infer that $p\left(\lambda \mid P_{0}\right)=p\left(\lambda \mid P_{1}\right)=p\left(\lambda \mid P_{2}\right)$, which states that mixed preparations corresponding to different values of the trit $t$ are not only indistinguishable at the operational level, but at the ontic level as well. Therefore, even if one knew $\lambda$, the posterior probabilities for $t=1, t=2$ and $t=3$ would be the same, that is, one would know nothing about the trit $t$. The argument so far can be summarized as follows: for preparationnoncontextual models, trit-obliviousness at the operational level implies trit-obliviousness at the ontic level. The ontic state $\lambda$ provides a classical encoding of $(t, b)$, but one that does not contain any information about $t$.

To finish the argument, we take note of all the functions of $t$ and $b$ that contain no information about $t 21$ These are equivalent, up to an affine transformation (i.e. up to a scalar multiple and an additive constant), to one of the following four functions

\begin{tabular}{c||c|c|c|c}
$t, b$ & $b$ & $c_{1}(t, b)$ & $c_{2}(t, b)$ & $c_{3}(t, b)$ \\
\hline 1,0 & 0 & 0 & 1 & 1 \\
1,1 & 1 & 1 & 0 & 0 \\
2,0 & 0 & 1 & 0 & 1 \\
2,1 & 1 & 0 & 1 & 0 \\
3,0 & 0 & 1 & 1 & 0 \\
3,1 & 1 & 0 & 0 & 1
\end{tabular}

where $c_{y}(t, b)$ is defined in Eq. (83). In an ontological model that respects preparation noncontextuality and the trit-obliviousness condition, the ontic state must be given by one of these four functions, that is, $p\left(\lambda \mid P_{t, b}\right)=$

21 In the sense that for any given value of the function $f(t, b)$, the conditional probability $p(t \mid f(t, b))=1 / 3$ for all $t$. $\delta_{\lambda, b}$ or $\delta_{\lambda, c_{1}(t, b)}$ or $\delta_{\lambda, c_{2}(t, b)}$ or $\delta_{\lambda, c_{3}(t, b)}$. Note that in each case, the ontic state space is a single bit 22 .

In the case of an ontological model wherein $\lambda=b$, the best the measurement device can do is to always output $b \oplus 1$ because with probability $2 / 3, y \neq t$ and $c_{y}(t, b)=$ $b \oplus 1$, while with probability $1 / 3, y=t$ and $c_{y}(t, b)=$ $b$. Thus, for this ontological model, the average success probability is $2 / 3$.

In the case of an ontological model wherein $\lambda=c_{1}(t, b)$, the best the measurement device can do is to output $c_{1}(t, b)$ when $y=1$ and $c_{1}(t, b) \oplus 1$ when $y \neq 1$. Note that $c_{1}(t, b) \oplus 1=c_{2}(t, b)$ for $2 / 3$ of the values of $t, b$ and $c_{1}(t, b) \oplus 1=c_{3}(t, b)$ also for $2 / 3$ of the values of $t, b$. (To see this, it suffices to take the negation of the $c_{1}(t, b)$ column of the table and compare it with the $c_{2}(t, b)$ and $c_{3}(t, b)$ columns.) So we see that this choice of output generates the right correlations $2 / 3$ of the time for $y \neq$ 1. Thus for this ontological model, the overall success probability is $7 / 9$.

By symmetry, the cases of $\lambda=c_{2}(t, b)$ and $\lambda=$ $c_{3}(t, b)$ also achieve a success probability of at most $7 / 9$. Therefore, the probability of success in a preparationnoncontextual ontological model is bounded above by $7 / 9$.

\section{B. Quantum case}

We now consider to what extent one can achieve the diachronic OS correlations in quantum theory. The following is a protocol that uses a single qubit. The three measurements correspond to the three Pauli operators $\hat{A}_{t}$ of Eq. (41) corresponding to directions equally spaced in an equatorial plane of the Bloch sphere. The positive and negative eigenvalues are mapped onto outputs $X=0$ and $X=1$ respectively. The preparation procedures $P_{t, 0}$ and $P_{t, 1}$ correspond to the two eigenstates of $\hat{A}_{t}$, with positive and negative eigenvalues mapped onto $b=0$ and $b=1$ respectively. We denote these states by the Hilbert space vectors $\left|\phi_{t, b}\right\rangle$. The Bloch sphere representation of these states and measurements is provided in Fig. 10. When $y=t$, the preparation corresponds to an eigenstate of the observable being measured, and the outcome $X$ equals the bit $b$. Thus, $X=c_{y}(t, b)$ with probability 1 in this case. When $y \neq t$, the probability of obtaining $X=b$ is $\left|\left\langle\phi_{t, b} \mid \phi_{y, b}\right\rangle\right|^{2}=\cos ^{2}(\pi / 3)=1 / 4$ while the probability of obtaining $X=b \oplus 1$ and thus $X=c_{y}(t, b)$ is $3 / 4$. We have $y \neq t$ in $2 / 3$ of cases, so that the overall probability of success is

$$
R_{3}^{\text {quantum }}=\frac{5}{6}
$$

22 It is not a triple of bits and therefore cannot specify the occupation numbers of each of the three boxes. In this sense, the narrative device of a three-box system cannot do justice to this version of the parable. We must think about the preparations and measurements more abstractly. 
Meanwhile, no information about $t$ can be obtained by any quantum measurement given that the mixtures associated with different values of $t$ are represented by the same density operator: $\frac{1}{2}\left|\phi_{0,0}\right\rangle\left\langle\phi_{0,0}\left|+\frac{1}{2}\right| \phi_{0,1}\right\rangle\left\langle\phi_{0,1}\left|=\frac{1}{2}\right| \phi_{1,0}\right\rangle\left\langle\phi_{1,0}\left|+\frac{1}{2}\right| \phi_{1,1}\right\rangle\left\langle\phi_{1,1}\right|$ $=\frac{1}{2}\left|\phi_{2,0}\right\rangle\left\langle\phi_{2,0}\left|+\frac{1}{2}\right| \phi_{2,1}\right\rangle\left\langle\phi_{2,1}\right|=\hat{\mathbf{1}} / 2$. Thus we have a violation of the noncontextuality inequality of Eq. (87).
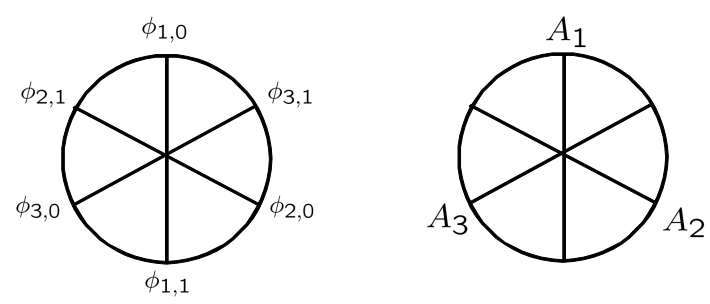

FIG. 10. Quantum states and observables used for proof of the impossibility of a preparation-noncontextual ontological model.

Note that the OS correlations are useful for achieving the following two-party secure computation, which is a kind of multiplexing. Let the two parties be called Alice and Bob. Alice has as input a trit $t \in\{1,2,3\}$ and a bit $b \in\{0,1\}$, each chosen uniformly at random. Bob has as input a trit $y \in\{1,2,3\}$ chosen uniformly at random. Bob outputs a bit $c$ and the goal of the task is for Bob to output $c=c_{y}(t, b)$, that is, Bob should output $b$ if $y=t$ and the negation of $b$ otherwise. Alice can send a system to Bob encoding information about her input, however there is a cryptographic constraint: no information about the trit $t$ can be transmitted to Bob, which is to say that the protocol must be trit-oblivious. This information-theoretic manner of characterizing the correlations provides a connection with the discussion of preparation noncontextuality found in Ref. [50].

\section{Justifying preparation noncontextuality by locality}

As discussed in Ref. [12], it is sometimes possible to justify an assumption of preparation noncontextuality using Bell's assumption of local causality 33. This is the case for the assumptions of preparation noncontextuality that appear in the derivation of the noncontextuality inequality of Eq. (87). It suffices to note that if one implements a measurement procedure on half of a correlated pair of systems and one conditions upon its outcome, then this procedure can also be considered a preparation procedure for the other half of the correlated pair. Indeed, given the separated pair of single-query 3-box systems considered in Sec. IVA, every measurement procedure $M_{t}$ on the 3-box system in Abydos chosen from $t \in\{1,2,3\}$ and yielding outcome $b \in\{0,1\}$ corresponds to a preparation procedure $P_{t, b}$ for the 3 -box system in Babylon. If $M_{t}$ is measured in Abydos but one does not condition on the outcome, then this corresponds to a preparation procedure $P_{t}$ of the system in Babylon. In this case, the probability of observing an outcome $X$ for a measurement of $M_{y}$ in Babylon given a preparation $P_{t, b}$ is precisely equal to the probability of observing an outcome $X$ for a measurement of $M_{y}$ in Babylon given an outcome $b$ for $M_{t}$ in Abydos. There is an isomorphism between the diachronic pair of single-query 3 -box systems and the separated pair.

Now suppose that the Abydosian and Babylonian measurements are space-like separated. In this case, the no-signaling constraint ensures that the choice of $t$ in Abydos cannot influence the outcome statistics of any measurement in Babylon and consequently that the three preparation procedures $P_{1}, P_{2}$ and $P_{3}$ are operationally equivalent, that is, $\forall M: p\left(X \mid M ; P_{1}\right)=p\left(X \mid M ; P_{2}\right)=$ $p\left(X \mid M ; P_{3}\right)$. This is the condition of trit-obliviousness.

Furthermore, an assumption of local causality implies that the choice of measurement in Abydos also cannot influence the distribution over ontic states for the 3-box system in Babylon. Denoting the ontic state of the Babylonian system by $\lambda$, local causality implies $p\left(\lambda \mid P_{1}\right)=$ $p\left(\lambda \mid P_{2}\right)=p\left(\lambda \mid P_{3}\right)$. But this is precisely the content of the assumption of preparation noncontextuality for the operationally equivalent procedures $P_{1}, P_{2}$ and $P_{3}$. Therefore local causality justifies this assumption.

This reasoning also shows that any local strategy for winning the prediction game for the separated pair of single-query 3-box systems implies a preparationnoncontextual strategy for winning the prediction game for the diachronic pair with the same winning probability 23 It follows that another way to derive the local bound of $7 / 9$ for the probability of achieving the OS correlations for the separated pair, Eq. (37), is to appeal to this implication and the fact that the optimal preparationnoncontextual strategy achieves a winning probability of $7 / 9$ for the diachronic pair, as shown in Eq. (87).

\section{JOINT MEASURABILITY OF POVMS}

As we showed early on, we cannot find a triple of projective measurements in quantum theory that are jointly measurable pairwise but not triplewise. However, not all measurements in quantum theory are projective. The most general measurement is one that is associated with a positive operator valued measure (POVM). A POVM is a set of operators $\left\{E_{X}: X \in S\right\}$ such that $E_{X} \geq 0$, and $\sum_{X} E_{X}=\hat{\mathbf{1}}$. The parameter $X$ labels the outcomes of the measurement, which we assume form a discrete

23 It has been shown that for every inequality on correlations between pairs of separated measurements that is implied by the assumption of a local ontological model, an equivalent inequality for the correlations between preparations and measurements is implied by the assumption of a preparation-noncontextual ontological model [51]. 
set. If the preparation procedure preceding the measurement is represented by the density operator $\rho$, then the probability of outcome $X$ is given by $\operatorname{Tr}\left(\rho E_{X}\right)$.

In this section, we consider the question of whether one could find a triple of non-projective measurements in quantum theory that are pairwise but not triplewise jointly measurable. As it turns out, this is indeed possible.

First, we adapt the definition of joint measurability to the case of POVMs. A pair of measurements associated with POVMs $\left\{E_{X_{1}}^{1}\right\}$ and $\left\{E_{X_{2}}^{2}\right\}$ are jointly measurable iff there exists a third POVM $\left\{F_{X_{1}, X_{2}}\right\}$ such that $E_{X_{1}}^{1}=$ $\sum_{X_{2}} F_{X_{1}, X_{2}}$ and $E_{X_{2}}^{2}=\sum_{X_{1}} F_{X_{1}, X_{2}}$. It is worth noting that the problem of mathematically characterizing jointly measurable observables when these are not projective is a subject of on-going research [13 16].

We will consider two examples of such triples of POVMs such that any pair can be implemented jointly, but the triple cannot. They both make use of noisy spin observables. The three measurements we consider, labelled by an integer $k \in\{1,2,3\}$, are associated with POVMs $\left\{E_{+}^{k}, E_{-}^{k}\right\}$

$$
E_{ \pm}^{k} \equiv \frac{1}{2} \hat{\mathbf{1}} \pm \frac{\eta}{2} \vec{\sigma} \cdot \hat{n}_{k}, \quad 0 \leq \eta \leq 1
$$

where $\vec{\sigma}=\left(\sigma_{x}, \sigma_{y}, \sigma_{z}\right)$ is the vector of Pauli spin operators, whilst $\hat{n}_{1}, \hat{n}_{2}$ and $\hat{n}_{3}$ are the three axes along which the spin is measured. Note that the POVM $\left\{E_{+}^{k}, E_{-}^{k}\right\}$ can be written as a convex combination of the projective spin measurement along $\hat{n}_{k}$ - associated with the projectors $\Pi_{ \pm}^{k} \equiv \frac{1}{2} \hat{\mathbf{1}} \pm \frac{1}{2} \vec{\sigma} \cdot \hat{n}_{k}$ - and the trivial measurement $\{\hat{\mathbf{1}} / 2, \hat{\mathbf{1}} / 2\}$. That is,

$$
E_{ \pm}^{k}=(1-\eta) \frac{1}{2} \hat{\mathbf{1}}+\eta \Pi_{ \pm}^{k}
$$

This is the sense in which we can consider $\left\{E_{+}^{k}, E_{-}^{k}\right\}$ with $\eta<1$ to be a noisy version of the observable $\vec{\sigma} \cdot \hat{n}_{k}$.

\section{A. Orthogonal spin axes}

Our first example of such a triple of nonprojective measurements uses noisy versions of spin operators along three orthogonal axes:

$$
\begin{aligned}
& \hat{n}_{1}=\hat{z} \equiv(0,0,1), \\
& \hat{n}_{2}=\hat{x} \equiv(1,0,0), \\
& \hat{n}_{3}=\hat{y} \equiv(0,1,0) .
\end{aligned}
$$

Proposition 8. The triple of measurements defined by Eqs. (92) and 94, that is, noisy spin observables along three orthogonal axes, are pairwise jointly measurable iff $\eta \leq 1 / \sqrt{2} \approx 0.707$, but triplewise jointly measurable iff $\eta \leq 1 / \sqrt{3} \approx 0.577$.

In other words, the condition $1 / \sqrt{3}<\eta \leq 1 / \sqrt{2}$ is necessary and sufficient for the triple to be pairwise jointly measurable but not triplewise jointly measurable.
This result is proven in Ref. [14], but for completeness, we provide an independent proof in Appendix [F For pedagogical reasons, we also provide a geometric picture in the Bloch sphere of the measurements that saturate these inequalities. To this end, defining the index set $\mathcal{I} \subseteq$ $\{1,2,3\}$, we introduce the (unnormalized Bloch) vectors

$$
\vec{m}_{\left\{X_{k}\right\}_{k \in \mathcal{I}}} \equiv \sum_{k \in \mathcal{I}} X_{k} \hat{n}_{k}
$$

where $X_{k} \in\{-1,+1\}$ and write the respective unit vectors as $\hat{m}_{\left\{X_{k}\right\}_{k \in \mathcal{I}}}$.

The POVM that measures a noisy spin observable along the $\hat{z}$-axis jointly with the one along the $\hat{x}$-axis and that saturates $\eta \leq 1 / \sqrt{2}$ is of the form

$$
\left\{F_{X_{1} X_{2}} \equiv \frac{1}{2} \Pi_{X_{1} X_{2}}\right\}
$$

where the projectors $\left\{\Pi_{X_{1} X_{2}}\right\}$ are associated with Bloch vectors $\left\{\hat{m}_{X_{1} X_{2}}\right\}$ forming the vertices of a square in the $\hat{z}-\hat{x}$ plane, depicted in Fig. 11. Coarse-graining over $X_{2}$ yields the POVM $\left\{F_{ \pm}^{1} \equiv \frac{1}{2} \hat{\mathbf{1}}+\frac{1}{2} \vec{\sigma} \cdot \vec{s}_{ \pm}^{1}\right\}$ where $\vec{s}_{ \pm}^{1} \equiv \pm \frac{1}{\sqrt{2}} \hat{z}$, which is to say, a measurement of the $\eta$ sharp spin observable along the $\hat{z}$ axis with $\eta=\frac{1}{\sqrt{2}}$, depicted in Fig. 11. Similarly, coarse-graining over $X_{1}$ yields noisy spin observable associated with Bloch vectors $\vec{s}_{ \pm}^{2}= \pm \frac{1}{\sqrt{2}} \hat{x}$, which is to say along the $\hat{x}$ axis with $\eta=\frac{1}{\sqrt{2}}$. Joint measurements of every other pair of spin axes are described similarly.
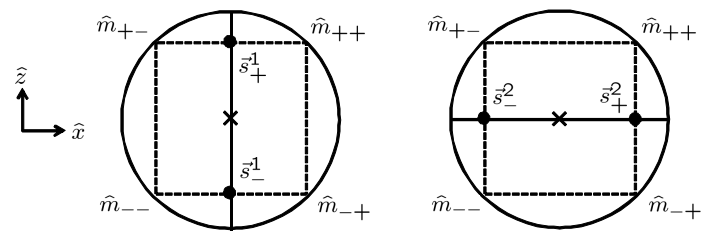

FIG. 11. Bloch sphere representation of the joint measurement of the noisy spin observables along the $\hat{x}$ and $\hat{z}$ axes.

The POVM that measures noisy spin observables along axes $\hat{z}, \hat{x}$ and $\hat{y}$ jointly and that saturates $\eta \leq 1 / \sqrt{3}$ is of the form $\left\{F_{X_{1} X_{2} X_{3}} \equiv \frac{1}{4} \Pi_{X_{1} X_{2} X_{3}}\right\}$ where the projectors $\left\{\Pi_{X_{1} X_{2} X_{3}}\right\}$ are associated with the Bloch vectors $\left\{\hat{m}_{X_{1} X_{2} X_{3}}\right\}$ forming the vertices of a cube, depicted in Fig. 12 Coarse-graining over $X_{2}$ and $X_{3}$ yields the $\mathrm{POVM}\left\{F_{ \pm}^{1} \equiv \frac{1}{2} \hat{\mathbf{1}}+\frac{1}{2} \vec{\sigma} \cdot \vec{s}_{ \pm}^{1}\right\}$ where $\vec{s}_{ \pm}^{1}= \pm \frac{1}{\sqrt{3}} \hat{z}$, which is to say an $\eta$-sharp spin observable along the $\hat{z}$ axis with $\eta=1 / \sqrt{3}$, also depicted in Fig. 12. Similarly, coarsegraining over $X_{1}$ and $X_{3}$ yields a noisy spin observable associated with Bloch vectors $\vec{s}_{ \pm}^{2}= \pm \frac{1}{\sqrt{3}} \hat{x}$, while coarse-graining over $X_{1}$ and $X_{2}$ yields one associated with $\vec{s}_{ \pm}^{3}= \pm \frac{1}{\sqrt{3}} \hat{y}$.

It is clear from these geometric representations that the reason there is a gap between the noise required for jointly measuring a pair and that required for jointly 

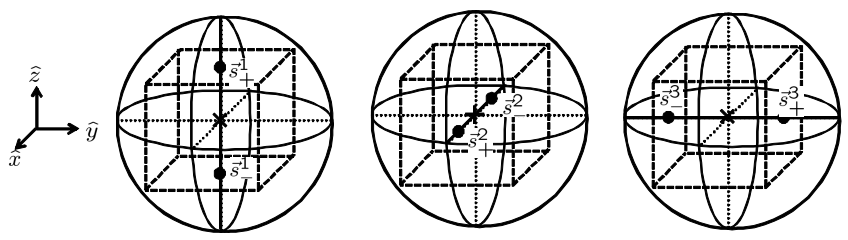

FIG. 12. Bloch sphere representation of the joint measurement of the noisy spin observables along the $\hat{x}, \hat{y}$ and $\hat{z}$ axes.

measuring the triple is that the length of the edge of a cube inscribed in a sphere is less than that of a square inscribed in an equatorial plane of that sphere.

Joint measurements of observables along orthogonal spin axes are not very useful for approximating the OS correlations. Indeed, defining the probability of obtaining anti-correlated outcomes when a pair of nonprojective measurements is implemented jointly, averaged uniformly over the three pairs,

$$
R_{3} \equiv \frac{1}{3} \sum_{j \neq k} p\left(X_{j} \neq X_{k} \mid \mathcal{M}_{j k} ; \lambda\right)
$$

we find the following result.

Proposition 9. For the triple of measurements defined by Eqs. (92) and 94), that is, noisy spin observables along three orthogonal axes, the quantum probability of anti-correlation when a pair is measured jointly, averaged uniformly over the three pairs is

$$
R_{3}^{\text {quantum }}=\frac{1}{2}
$$

(independent of the quantum state).

Proof. The intuitive reason is that each pair of spin observables is unbiased. More precisely, if we coarse-grain over the effects in the joint POVM $\left\{F_{X_{1} X_{2}}\right\}$ of Eq. (96) with outcomes corresponding to anti-correlation, we get

$$
F_{+-}+F_{-+}=\frac{1}{2} \hat{\mathbf{1}} .
$$

Therefore, for all quantum states, the probability of finding anti-correlated results is $1 / 2$.

There is consequently no bias towards anti-correlation and therefore this triple of measurements is not helpful for approximating the OS correlations.

\section{B. Trine spin axes}

Our second example consists of noisy versions of spin observables along three axes equally separated in a plane (i.e. separated by a trine or an angle of $120^{\circ}$ ):

$$
\begin{aligned}
& \hat{n}_{1}=(0,0,1) \\
& \hat{n}_{2}=\left(\frac{\sqrt{3}}{2}, 0,-\frac{1}{2}\right) \\
& \hat{n}_{3}=\left(-\frac{\sqrt{3}}{2}, 0,-\frac{1}{2}\right) .
\end{aligned}
$$

These are depicted in Fig. 13

Proposition 10. The triple of measurements defined by Eqs. (92) and (100), that is, noisy spin observables along three equally-spaced axes in a plane, are pairwise jointly measurable if $\eta \leq \sqrt{3}-1 \simeq 0.73205$, but triplewise jointly measurable only if $\eta \leq 2 / 3$.

In other words, the condition $2 / 3<\eta \leq \sqrt{3}-1$ is sufficient for the triple to be pairwise jointly measurable but not triplewise jointly measurable.

Again, the proof is provided in Appendix $\mathrm{F}$, but we can understand the result geometrically. The trine directions $\hat{n}_{1}, \hat{n}_{2}$ and $\hat{n}_{3}$ of Eq. (100) are indicated in Fig. 13, The POVM that measures a noisy spin observable along the $\hat{n}_{1}$-axis jointly with the one along the $\hat{n}_{3}$-axis and that saturates $\eta \leq \sqrt{3}-1$ is of the form

$$
\left\{F_{X_{1} X_{2}} \equiv w_{X_{1} X_{2}} \Pi_{X_{1} X_{2}}\right\}
$$

where

$$
\begin{gathered}
w_{++}=w_{--}=\frac{1}{\sqrt{3}+1} \\
w_{+-}=w_{-+}=\frac{\sqrt{3}}{\sqrt{3}+1},
\end{gathered}
$$

and where the projectors $\left\{\Pi_{X_{1} X_{2}}\right\}$ are associated with Bloch vectors $\left\{\hat{m}_{X_{1} X_{2}}\right\}$ forming the vertices of a square, depicted in Fig. 13. Coarse-graining over $X_{2}$ yields the POVM $\left\{F_{ \pm}^{1} \equiv \frac{1}{2} \hat{\mathbf{1}}+\frac{1}{2} \vec{\sigma} \cdot \vec{s}_{ \pm}^{1}\right\}$ with $\vec{s}_{ \pm}^{1}= \pm(\sqrt{3}-1) \hat{n}_{1}$ depicted in Fig. 13. Similarly, coarse-graining over $X_{1}$ yields a noisy spin observable associated with Bloch vectors $\vec{s}_{ \pm}^{3}= \pm(\sqrt{3}-1) \hat{n}_{3}$. Joint measurements of every other pair of spin axes are described similarly.
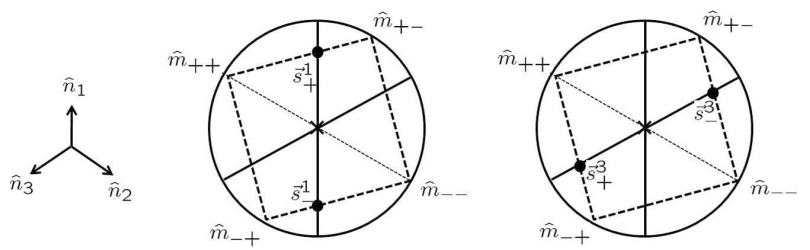

FIG. 13. Bloch sphere representation of the joint measurement of the noisy spin observables along trine axes $\hat{n}_{1}$ and $\hat{n}_{3}$.

The POVM that measures noisy spin observables along axes $\hat{n}_{1}, \hat{n}_{2}$ and $\hat{n}_{3}$ jointly and that saturates $\eta \leq 2 / 3$ 
is of the form $\left\{F_{X_{1} X_{2} X_{3}} \equiv w_{X_{1} X_{2} X_{3}} \Pi_{X_{1} X_{2} X_{3}}\right\}$ where $w_{+++}=w_{---}=0$ (implying that one never obtains a triplewise coincidence in the joint measurement) while $w_{+--}=w_{-++}=w_{+-+}=w_{-+-}=w_{--+}=w_{++-}=$ $1 / 3$ and where the projectors $\left\{\Pi_{X_{1} X_{2} X_{3}}\right\}$ are associated with Bloch vectors $\left\{\hat{m}_{X_{1} X_{2} X_{3}}\right\}$ forming the vertices of a hexagon for the six values of $X_{1} X_{2} X_{3}$ such that $w_{X_{1} X_{2} X_{3}} \neq 0$, as depicted in Fig. 14] Coarse-graining over $X_{2}$ and $X_{3}$ yields the POVM $\left\{F_{ \pm}^{1} \equiv \frac{1}{2} \hat{\mathbf{1}}+\frac{1}{2} \vec{\sigma} \cdot \vec{s}_{ \pm}^{1}\right\}$ where $\vec{s}_{ \pm}^{1}= \pm \frac{2}{3} \hat{n}_{1}$, depicted in Fig. 14. Similarly, coarsegraining over $X_{1}$ and $X_{3}$ yields a noisy spin observable associated with Bloch vectors $\vec{s}_{ \pm}^{2}= \pm \frac{2}{3} \hat{n}_{2}$, while coarse-graining over $X_{1}$ and $X_{2}$ yields one associated with $\vec{s}_{ \pm}^{3}= \pm \frac{2}{3} \hat{n}_{3}$. Note that, unlike the three previous examples, the Bloch-directions of the fine-grained (saturating) POVM elements coincide with the Bloch-directions of the coarse-grained POVM elements. This is a peculiarity of geometry, and is a feature also seen in the dual problem of identifying pure-state ensembles that saturate the bounds of so-called EPR-steering inequalities [52].

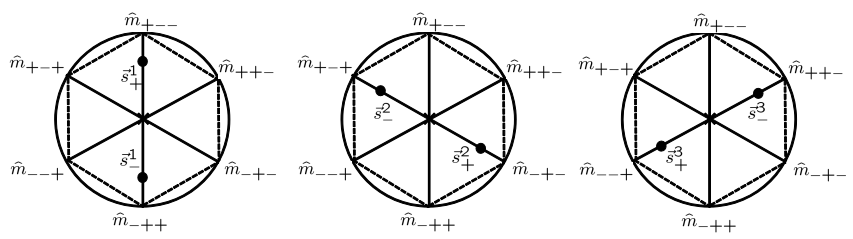

FIG. 14. Bloch sphere representation of the joint measurement of the noisy spin observables along trine axes $\hat{n}_{1}, \hat{n}_{2}$ and $\hat{n}_{3}$.

Given the discussion in Sec.IVB, one might expect the trine spin observables to instantiate a better approximation of the OS correlations. Indeed, we have the following proposition that supports this intuition.

Proposition 11. For the triple of measurements defined by Eqs. (92) and (100), that is, a triple of noisy spin observables along trine axes, the quantum probability of anti-correlation when a pair is measured jointly, averaged uniformly over the three pairs is

$$
R_{3}^{\text {quantum }}=\frac{\sqrt{3}}{\sqrt{3}+1} \simeq 0.63397 .
$$

(independent of the quantum state).

Proof. If, in the joint measurement of Eq. (101), we coarse-grain the two effects that correspond to anticorrelation, we obtain

$$
F_{+-}+F_{-+}=\frac{\sqrt{3}}{\sqrt{3}+1} \hat{\mathbf{1}}
$$

from which the result follows trivially.

Can we explain this degree of anti-correlation within a generalized-noncontextual ontological model? Given that the measurements involved are nonprojective, we need not represent them as assigning deterministic outcomes for every ontic state. Indeed, as discussed in Sec.IID for nonprojective measurements, one is not warranted in assuming outcome determinism. It follows that the bound of $2 / 3$ on the probability of anti-correlation, Eq. (12), which we derived under the assumption of measurements being projective, need not apply. Conceivably, the bound implied by generalized noncontextuality could be smaller for nonprojective measurements, and the quantum degree of anti-correlation might therefore still violate it. As it turns out however, the bound is actually larger for nonprojective measurements, and therefore the quantum degree of anti-correlation is entirely consistent with an ontological model that is measurementnoncontextual and outcome-deterministic for projective measurements. We show this now.

\section{Generalized-noncontextual models for joint measurements of POVMs}

Each measurement that is modeled by a POVM of the form of Eq. (92) can be considered as a convex combination of a projective measurement and a measurement of the trivial two-outcome POVM $\{\hat{\mathbf{1}} / 2, \hat{\mathbf{1}} / 2\}$, as seen in Eq. [93). In Ref. [18], it is proven that within any ontological model, the response function that represents a convex combination of measurement procedures is simply the convex combination of the associated response functions. Ref. 18] also contains a proof that within a measurement-noncontextual model, the response function that represents each outcome of the trivial twooutcome POVM $\{\hat{\mathbf{1}} / 2, \hat{\mathbf{1}} / 2\}$ is the uniform function $1 / 2$, i.e., regardless of the value of $\lambda$ in the ontological model, the two outcomes occur with equal probability. We also recall from Sec. IIE that in models of quantum theory, preparation noncontextuality implies outcome determinism for projective measurements. From these facts, we obtain the following result.

Lemma 12. In an ontological model that is generalizednoncontextual, the response function for the $\eta$-sharp spin observable of Eq. (92), denoted by $\mathcal{M}_{k}$, is

$$
p\left(X_{k} \mid \mathcal{M}_{k} ; \lambda\right)=\eta\left[X_{k}(\lambda)\right]+(1-\eta)\left(\frac{1}{2}[0]+\frac{1}{2}[1]\right),
$$

where $[X(\lambda)]$ denotes the response function $p(X \mid \lambda)=1$ if $X=X(\lambda)$ and 0 otherwise.

This yields a strong constraint on the response function for the joint measurement, denoted $\mathcal{M}_{12}$, of $\eta$-sharp spin observables along distinct axes. The joint response function $p\left(X_{1}, X_{2} \mid \mathcal{M}_{12} ; \lambda\right)$ must yield $p\left(X_{1} \mid \mathcal{M}_{1} ; \lambda\right)$ when averaged over $X_{2}$ and $p\left(X_{2} \mid \mathcal{M}_{2} ; \lambda\right)$ when averaged over $X_{1}$. The most general form that can recover these marginals 
is

$$
\begin{aligned}
p\left(X_{1}, X_{2} \mid \mathcal{M}_{12} ; \lambda\right) & =\alpha\left[X_{1}(\lambda)\right]\left[X_{2}(\lambda)\right] \\
& +\beta\left[X_{1}(\lambda)\right]\left(\frac{1}{2}[0]+\frac{1}{2}[1]\right) \\
& +\gamma\left(\frac{1}{2}[0]+\frac{1}{2}[1]\right)\left[X_{2}(\lambda)\right] \\
& +\delta\left(\frac{1}{2}[0][0]+\frac{1}{2}[1][1]\right) \\
& +\varepsilon\left(\frac{1}{2}[0][1]+\frac{1}{2}[1][0]\right) .
\end{aligned}
$$

where the marginals are

$$
\begin{aligned}
p\left(X_{1} \mid M_{12} ; \lambda\right) & =(\alpha+\beta)\left[X_{1}(\lambda)\right] \\
& +(\gamma+\delta+\varepsilon)\left(\frac{1}{2}[0]+\frac{1}{2}[1]\right) \\
p\left(X_{2} \mid M_{12} ; \lambda\right) & =(\alpha+\gamma)\left[X_{2}(\lambda)\right] \\
& +(\beta+\delta+\varepsilon)\left(\frac{1}{2}[0]+\frac{1}{2}[1]\right),
\end{aligned}
$$

so that we require

$$
\begin{aligned}
\alpha+\beta & =\alpha+\gamma=\eta, \\
\gamma+\delta+\varepsilon & =\beta+\delta+\varepsilon=1-\eta .
\end{aligned}
$$

We infer that $\beta=\gamma$.

In order to give the model the best chance of reproducing the operational statistics, we consider what values of $\alpha, \beta, \gamma, \delta$ and $\varepsilon$ achieve the largest possible amount of anti-correlation. The $\delta$ terms always yields correlation, while the $\beta$ and $\gamma$ terms yield correlation as often as anti-correlation. Only the $\alpha$ and $\varepsilon$ terms can have anti-correlation more frequently than correlation. Thus, to maximize the amount of anti-correlation, one sets $\beta=\gamma=\delta=0$. It then follows that $\alpha=\eta$ and $\varepsilon=1-\eta$.

The same reasoning applies for the joint measurements of $\mathcal{M}_{1}$ and $\mathcal{M}_{3}$ and of $\mathcal{M}_{2}$ and $\mathcal{M}_{3}$, so that for all $i, j \in$ $\{1,2,3\}$ such that $i \neq j$,

$$
\begin{aligned}
p\left(X_{i}, X_{j} \mid \mathcal{M}_{i j} ; \lambda\right) & =\eta\left[X_{i}(\lambda)\right]\left[X_{j}(\lambda)\right] \\
& +(1-\eta)\left(\frac{1}{2}[0][1]+\frac{1}{2}[1][0]\right) .
\end{aligned}
$$

The question then arises of how much anti-correlation one can have on average for a pair of measurements (assuming the pair is chosen uniformly at random), that is, what is the upper bound on $R_{3}$ of Eq. (97)? For every $\lambda$, at most two out of the three products $\left[X_{1}(\lambda)\right]\left[X_{2}(\lambda)\right],\left[X_{1}(\lambda)\right]\left[X_{3}(\lambda)\right]$ and $\left[X_{2}(\lambda)\right]\left[X_{3}(\lambda)\right]$ can yield anti-correlation, so the probability of anticorrelation for the $\eta$ term is at most 2/3. Meanwhile, the $1-\eta$ term always yield anti-correlation. Therefore,

$$
R_{3} \leq \eta\left(\frac{2}{3}\right)+(1-\eta)=1-\frac{\eta}{3} .
$$

One might have expected that the ability to add noise to the response function in the ontological model would not help explain a high degree of anti-correlation, but such an expectation fails to take into account the fact that the noise can itself be anti-correlated and thereby explain more anti-correlation in the statistics. Thus rather than only being able to explain a probability of anticorrelation of $2 / 3$ in a generalized-noncontextual model, we can explain a probability of anti-correlation of $1-\frac{\eta}{3}$ which is always greater than $2 / 3$ because $\eta \leq 1$. For instance, for $\eta=1 / \sqrt{2}$, the upper bound on $R_{3}$ is $1-1 /(3 \sqrt{2}) \simeq 0.76430$, while for $\eta=\sqrt{3}-1$, it is $(4-\sqrt{3}) / 3 \simeq 0.75598$.

Because the degree of anti-correlation we found in quantum theory was less than $2 / 3$ in both examples, there is no problem providing a generalizednoncontextual model. More precisely, the degree of quantum anti-correlation obtained in the example with orthogonal spin axes can be explained noncontextually because $R_{3}^{\text {quantum }}=1 / 2<0.76430$, and the degree obtained in the example with the trine spin axes can be explained noncontextually because $R_{3}^{\text {quantum }}=0.63397<$ 0.75598 .

Is it the case that for all triples of nonprojective quantum measurements that can be implemented pairwise but not triplewise, the strength of anti-correlations can be explained by a generalized-noncontextual ontological model? The question remains open, but we expect a positive answer.

\section{CONCLUDING REMARKS}

There has been a lot of work in recent years on "foils to quantum theory", operational theories that one studies not primarily as competitors to quantum theory, but as useful tools for getting a handle on the principles underlying it. Only by situating quantum theory in a landscape of possible theories does it make sense to speak of the principles that pick it out, to answer Wheeler's question: "how come the quantum?". Specker's parable provides an interesting new kind of foil, because the kind of complementarity it exhibits - three measurements that can be implemented jointly pairwise but not triplewise - is something that is not found among projective measurements in quantum theory. This prompts the question: why does quantum theory not have this sort of complementarity? It might be interesting, for instance, to deduce the information-processing power of a foil theory incorporating such correlations. Furthermore, even if we consider a kind of complementarity that can be accommodated in quantum theory, such as five measurements that can be measured in adjacent pairs, there is an interesting question about why the correlations exhibited by quantum theory are not stronger. Why is quantum theory not more contextual or more nonlocal [17, 53 62]? The same sort of question arises for quantum examples of triples of nonprojective measurements that can be imple- 
mented pairwise but not triplewise. Why can these not yield the strength of anti-correlations required to obtain a no-go theorem for generalized noncontextuality? We hope that these questions might provide a new angle on the problem of deriving the structure of quantum theory from within a landscape of operational foil theories.

\section{ACKNOWLEDGEMENT}

This project was inspired by Ernst Specker's talk at the workshop "Information Primitives and Laws of Nature" which took place at ETH, Zürich in May 2008. On the topic of the joint measurability of POVMs, we thank Robin Blume-Kohout for a motivating discussion and David Pegg for helpful comments. On the topic of nonlocal OS correlations, we acknowledge useful discussions with Ben Toner and Jean-Daniel Bancal. We also thank Allen Stairs for comments on a draft of this article and Lucien Hardy for bringing Ref. [40] to our attention and for suggesting the connection between Specker's parable and the failure of transitivity of implication in proofs of nonlocality. Finally, we thank Adán Cabello for pointing out the connection between our Kochen-Specker proof based on the failure of transitivity of implication and Clifton's proof. YCL and HMW acknowledge funding from the Australian Research Council. Part of this work was conducted during visits by YCL and HMW to Perimeter Institute, and RWS to Australia, through the PIAF (Perimeter Institute Australia Foundations) collaboration. Perimeter Institute is supported by the Government of Canada through Industry Canada and by the Province of Ontario through the Ministry of Research and Innovation. YCL was also supported by the Swiss NCCR "Quantum Photonics" and the European ERCAG QORE.

\section{Appendix A: Explicit form of OS correlations in the double-query, 3-box system}

Perfect negative correlation in the outcomes of the joint observables $\mathcal{M}_{12}, \mathcal{M}_{13}$ and $\mathcal{M}_{23}$ constrains their statistics to be of the form

$$
\begin{aligned}
& p\left(0,1 \mid \mathcal{M}_{12} ; \mathcal{P}_{*}\right)=q_{12}, \quad p\left(1,0 \mid \mathcal{M}_{12} ; \mathcal{P}_{*}\right)=1-q_{12}, \\
& p\left(0,1 \mid \mathcal{M}_{13} ; \mathcal{P}_{*}\right)=q_{13}, \quad p\left(1,0 \mid \mathcal{M}_{13} ; \mathcal{P}_{*}\right)=1-q_{13}, \\
& p\left(0,1 \mid \mathcal{M}_{23} ; \mathcal{P}_{*}\right)=q_{23}, \quad p\left(1,0 \mid \mathcal{M}_{23} ; \mathcal{P}_{*}\right)=1-q_{23}
\end{aligned}
$$

for $0 \leq q_{12}, q_{13}, q_{23} \leq 1$. This fixes the statistics for the individual measurements $\mathcal{M}_{1}, \mathcal{M}_{2}$ and $\mathcal{M}_{3}$ through the marginals of Eq. A1. Specifically,

$$
\begin{aligned}
p\left(0 \mid \mathcal{M}_{1} ; \mathcal{P}_{*}\right) & =\sum_{X_{2}} p\left(0, X_{2} \mid \mathcal{M}_{12} ; \mathcal{P}_{*}\right)=q_{12}, \\
& =\sum_{X_{3}} p\left(0, X_{3} \mid \mathcal{M}_{13} ; \mathcal{P}_{*}\right)=q_{13}, \\
p\left(0 \mid \mathcal{M}_{2} ; \mathcal{P}_{*}\right) & =\sum_{X_{1}} p\left(X_{1}, 0 \mid \mathcal{M}_{12} ; \mathcal{P}_{*}\right)=1-q_{12}, \\
& =\sum_{X_{3}} p\left(0, X_{3} \mid \mathcal{M}_{23} ; \mathcal{P}_{*}\right)=q_{23}, \\
p\left(0 \mid \mathcal{M}_{3} ; \mathcal{P}_{*}\right) & =\sum_{X_{1}} p\left(X_{1}, 0 \mid \mathcal{M}_{13} ; \mathcal{P}_{*}\right)=1-q_{13}, \\
& =\sum_{X_{2}} p\left(X_{2}, 0 \mid \mathcal{M}_{23} ; \mathcal{P}_{*}\right)=1-q_{23}
\end{aligned}
$$

All together, we find that we must have

$$
q_{12}=q_{13}=q_{23}=\frac{1}{2}
$$

which implies that the correlations are of the form of Eq. (5).

\section{Appendix B: Proof of theorem 6}

Here, we provide the proof of theorem 6 as follows.

Proof. By measurement noncontextuality, the response function depends only on the equivalence class of a measurement procedure. By outcome determinism, the response function for every measurement $\mathcal{M}_{S}$ is deterministic, so that $p\left(X_{S} \mid \mathcal{M}_{S} ; \lambda\right) \in\{0,1\}$. In particular, this is true for singleton sets. It follows that

$$
p\left(X_{S} \mid \mathcal{M}_{S} ; \lambda\right)=\prod_{s \in S} p\left(X_{s} \mid \mathcal{M}_{s} ; \lambda\right)
$$

We can then define a joint distribution $p\left(X_{1} \ldots X_{N} \mid \lambda\right)$ yielding the correct marginals by the product of the single measurement response functions,

$$
p\left(X_{1} \ldots X_{N} \mid \lambda\right) \equiv \prod_{s=1}^{N} p\left(X_{s} \mid \mathcal{M}_{s} ; \lambda\right) .
$$

By assumption of the empirical adequacy of the ontological model, there exists a distribution $p(\lambda \mid \mathcal{P})$ for all $\mathcal{P}$, such that

$$
\int d \lambda p\left(X_{S} \mid \mathcal{M}_{S}, \lambda\right) p(\lambda \mid \mathcal{P})=p\left(X_{S} \mid \mathcal{M}_{S} ; \mathcal{P}\right)
$$

Using $p(\lambda \mid \mathcal{P})$, we can define

$$
p\left(X_{1} \ldots X_{N} \mid \mathcal{P}\right)=\int d \lambda p\left(X_{1} \ldots X_{N} \mid \lambda\right) p(\lambda \mid \mathcal{P})
$$


which has marginal on $X_{S}$ of

$$
\begin{aligned}
p\left(X_{S} \mid \mathcal{P}\right) & =\sum_{X_{\nu}: \nu \notin S} p\left(X_{1} \ldots X_{N} \mid \mathcal{P}\right) \\
& =\int d \lambda \sum_{X_{\nu}: \nu \notin S} p\left(X_{1} \ldots X_{N} \mid \lambda\right) p(\lambda \mid \mathcal{P}) \\
& =\int d \lambda p\left(X_{S} \mid \mathcal{M}_{S}, \lambda\right) p(\lambda \mid \mathcal{P}) \\
& =p\left(X_{S} \mid \mathcal{M}_{S} ; \mathcal{P}\right)
\end{aligned}
$$

We have therefore shown that $p\left(X_{1} \ldots X_{N} \mid \mathcal{P}\right)$ is a joint distribution whose marginals yield the operational statistics of all measurements.

\section{Appendix C: Maximal quantum violation of the $n$-box-set Klyachko-type Kochen-Specker inequality}

To see that $\mathcal{S}_{n}^{\text {quantum }}$ given in Eq. (27) is indeed the strongest possible quantum violation of inequality (22), it suffices to consider the following polynomial of Hermitian operators

$$
\hat{\mathcal{B}}_{n}=\sum_{a=1}^{n} \bar{X}_{a} \bar{X}_{a \oplus 1}
$$

and note that for arbitrary Hermitian operators satisfying the commutation relation $\left[\bar{X}_{a}, \bar{X}_{a \oplus 1}\right]=0$, we have

$$
\begin{aligned}
& \hat{\mathcal{B}}_{n}-n\left(1-\frac{4 \cos \frac{\pi}{n}}{1+\cos \frac{\pi}{n}}\right) \hat{\mathbf{1}} \\
= & \frac{1}{4}\left(2-\sec \frac{\pi}{n}\right) \sum_{a=1}^{n}\left[\hat{\mathbf{1}}-\left(\bar{X}_{a}\right)^{2}\right]+\frac{1}{4} \sum_{a=1}^{n}\left[\hat{\mathbf{1}}-\left(\bar{X}_{a} \bar{X}_{a \oplus 1}\right)^{2}\right] \\
& +\frac{1}{4} \sec \frac{\pi}{n} \sum_{a=1}^{n} \bar{X}_{a} \bar{X}_{a \oplus 2}\left[\hat{\mathbf{1}}-\left(\bar{X}_{a \oplus 1}\right)^{2}\right] \\
& +\frac{1}{4 n}\left(1+\sec \frac{\pi}{n}\right) v_{0}^{\dagger} v_{0}+\frac{1}{n} \sum_{k=1,2} \sum_{j=1}^{n} \lambda_{k j} v_{k j}^{\dagger} v_{k j}, \quad \text { (C2) }
\end{aligned}
$$

where

$$
\begin{aligned}
& v_{0}=n\left(3-2 \sec ^{2} \frac{\pi}{2 n}\right) \hat{\mathbf{1}}+\sum_{a=1}^{n} \bar{X}_{a} \bar{X}_{a \oplus 1}, \\
& v_{1 j}=\sum_{a=1}^{n} \omega_{n}^{j a} \bar{X}_{a}, \quad v_{2 j}=\sum_{a=1}^{n} \omega_{n}^{j a} \bar{X}_{a} \bar{X}_{a \oplus 1}
\end{aligned}
$$

$\omega_{n}=\mathrm{e}^{-i 2 \pi / n}$ is the $n$-th root of unity, $\lambda_{2 n}=0$, and

$$
\begin{aligned}
& \lambda_{1 j}=\left(1+\cos \frac{2 \pi j}{n} \sec \frac{\pi}{n}\right) \sin ^{2} \frac{\pi j}{n}, \quad j=1, \ldots, n, \\
& \lambda_{2 j}=\frac{1}{4}\left(1+\cos \frac{2 \pi j}{n} \sec \frac{\pi}{n}\right), \quad j=1, \ldots, n-1 .
\end{aligned}
$$

It is straightforward to check that $\lambda_{1 j}$ and $\lambda_{2 j}$ are nonnegative for all $j$. Thus, for dichotomic Hermitian observables that also satisfy $\left(\bar{X}_{a}\right)^{2}=\hat{\mathbf{1}}$, the right-hand-side of
Eq. (C2) become a sum of non-negative Hermitian operators and hence non-negative. As a result, the smallest eigenvalue of $\hat{\mathcal{B}}_{n}$ is lower bounded by $n\left(1-\frac{4 \cos \frac{\pi}{n}}{1+\cos \frac{\pi}{n}}\right)$, therefore making $\mathcal{S}_{n}^{\text {quantum }}$ given in Eq. (27) the strongest possible quantum violation of inequality (22).

\section{Appendix D: Explicit form of correlations in the separated pair of single-query 3-box systems}

Here, we will give a simple proof that after taking into account of the no-signaling condition, the nonlcoal OS correlations have to take the form of Eq. (35).

First, note that by virtue of satisfying Eq. (33), the nonlocal OS correlations may be written as

$$
\begin{array}{r}
\forall a \neq b: \quad p\left(0,1 \mid \mathcal{M}_{a}, \mathcal{M}_{b} ; \mathcal{P}_{*}\right)=q_{a b} \\
p\left(1,0 \mid \mathcal{M}_{a}, \mathcal{M}_{b} ; \mathcal{P}_{*}\right)=1-q_{a b} \\
\forall a=b: \quad p\left(0,0 \mid \mathcal{M}_{a}, \mathcal{M}_{b} ; \mathcal{P}_{*}\right)=q_{a b} \\
p\left(1,1 \mid \mathcal{M}_{a}, \mathcal{M}_{b} ; \mathcal{P}_{*}\right)=1-q_{a b},
\end{array}
$$

where $0 \leq q_{a b} \leq 1$. These joint probabilities are depicted in Table 【

\begin{tabular}{|c|c|cc|cc|cc|}
\hline & & \multicolumn{2}{|c|}{$B_{1}$} & \multicolumn{2}{c|}{$B_{2}$} & \multicolumn{2}{c|}{$B_{3}$} \\
\hline & & 0 & 1 & 0 & 1 & 0 & 1 \\
\hline \multirow{2}{*}{$A_{1}$} & 0 & $q_{11}$ & 0 & 0 & $q_{12}$ & 0 & $q_{13}$ \\
& 1 & 0 & $1-q_{11}$ & $1-q_{12}$ & 0 & $1-q_{13}$ & 0 \\
\hline \multirow{2}{*}{$A_{2}$} & 0 & 0 & $q_{21}$ & $q_{22}$ & 0 & 0 & $q_{23}$ \\
& 1 & $1-q_{21}$ & 0 & 0 & $1-q_{22}$ & $1-q_{23}$ & 0 \\
\hline \multirow{2}{*}{$A_{3}$} & 0 & 0 & $q_{31}$ & 0 & $q_{32}$ & $q_{33}$ & 0 \\
& 1 & $1-q_{31}$ & 0 & $1-q_{32}$ & 0 & 0 & $1-q_{33}$ \\
\hline
\end{tabular}

TABLE I. Joint conditional probability distributions $p\left(A_{a}, B_{b} \mid \mathcal{M}_{a}, \mathcal{M}_{b} ; \mathcal{P}\right)$ of Eq. (D1) for all pairs of values of $a$ and $b$. Along the horizontal (vertical) are the three choices of measurement on the $B(A)$ wing together with the two outcomes for each.

Now we consider the consequences of the no-signaling conditions of Eq. (34). From the independence on $b$ of $\sum_{B_{b}} p\left(A_{a}, B_{b} \mid \mathcal{M}_{a}, \mathcal{M}_{b} ; \mathcal{P}\right)$, we deduce that

$$
q_{a 1}=q_{a 2}=q_{a 3},
$$

implying that the joint distributions can be made to depend on just three parameters, which we will denote by $s_{a}=q_{a b}$. It then follows from the independence on $a$ of $\sum_{A_{a}} p\left(A_{a}, B_{b} \mid \mathcal{M}_{a}, \mathcal{M}_{b} ; \mathcal{P}\right)$ that

$$
s_{a}=1-s_{a},
$$

which implies that $s_{a}=\frac{1}{2}$ for all $a$, and therefore $q_{a b}=\frac{1}{2}$ for all $a, b$. It follows that the nonlocal OS correlations must be of the form given in Eq. (35) if they are to be non-signaling. 


\section{Appendix E: Maximum quantum violation of the $n$-box-set Bell-Mermin inequality}

In general, the problem of determining the maximal quantum violation of a Bell inequality is highly nontrivial (see, for example, Refs. 23, 63, 64 and references therein). Here, we will show that $R_{n}^{\text {quantum }}$ defined in Eq. (46) is indeed the maximal winning probability, $c f$. Eq. (43), allowed in quantum mechanics. To this end, it suffices to show that the winning probability $R_{n}$ is upper bounded by $R_{n}^{\text {quantum }}$ in quantum theory. For convenience, we will show this in terms of

$$
\mathcal{S}_{n} \equiv \sum_{a=1}^{n}\left\langle\bar{A}_{a} \bar{B}_{a}\right\rangle-\sum_{a, b: b=a \oplus 1}\left\langle\bar{A}_{a} \bar{B}_{b}\right\rangle-\sum_{a, b: a=b \oplus 1}\left\langle\bar{A}_{a} \bar{B}_{b}\right\rangle,
$$

which can be re-expressed as:

$$
\mathcal{S}_{n}=6 n\left(R_{n}-\frac{1}{2}\right)
$$

using Eq. (38).

Now, consider the Bell operator [65] corresponding to above expression defining $S_{n}$ :

$$
\hat{\mathcal{B}}_{\mathrm{NLOS}}^{[n]}=\sum_{a=1}^{n} \bar{A}_{a} \bar{B}_{a}-\sum_{a, b: b=a \oplus 1} \bar{A}_{a} \bar{B}_{b}-\sum_{a, b: a=b \oplus 1} \bar{A}_{a} \bar{B}_{b} .
$$

Following a procedure very similar to that described in Sec. III of Ref. [66] (see also Ref. 23]), one finds that for arbitrary Hermitian observables $\left\{\bar{A}_{a}\right\}_{a=1}^{n}$ and $\left\{\bar{B}_{b}\right\}_{b=1}^{n}$ satisfying $\left[\bar{A}_{a}, \bar{B}_{b}\right]=0$,

$$
\begin{aligned}
& n \lambda_{\frac{n+1}{2}} \hat{\mathbf{1}}-\hat{\mathcal{B}}_{\mathrm{NLOS}}^{[n]} \\
= & \frac{1}{2} \sum_{a=1}^{n}\left[\left(\lambda_{\frac{n+1}{2}}+\lambda_{a}\right) v_{a-}^{\dagger} v_{a-}+\left(\lambda_{\frac{n+1}{2}}-\lambda_{a}\right) v_{a+}^{\dagger} v_{a+}\right] \\
+ & \frac{1}{2} \lambda_{\frac{n+1}{2}}\left\{\sum_{a=1}^{n}\left[\hat{\mathbf{1}}-\left(\bar{A}_{a}\right)^{2}\right]+\sum_{b=1}^{n}\left[\hat{\mathbf{1}}-\left(\bar{B}_{b}\right)^{2}\right]\right\}, \quad(\mathrm{E} 4)
\end{aligned}
$$

where

$v_{a \pm}=\frac{1}{\sqrt{2 n}} \sum_{k=1}^{n} \omega_{n}^{a k}\left(\bar{A}_{k} \pm \bar{B}_{k}\right), \quad \lambda_{a}=1-2 \cos \frac{2 \pi}{n} a$,

and $\omega_{n}=\mathrm{e}^{-i 2 \pi / n}$.

It is easy to verify that

$$
\max _{a \in\{1,2, \ldots, n\}} \lambda_{a}=\lambda_{\frac{n+1}{2}}=4 \cos ^{2} \frac{\pi}{2 n}-1 .
$$

Thus, Eq. (E4) implies that whenever the constraints $\left(\bar{A}_{a}\right)^{2}=\hat{\mathbf{1}}$ and $\left(\bar{B}_{b}\right)^{2}=\hat{\mathbf{1}}$ are satisfied for all $a, b \in$ $\{1,2, \ldots, n\}$, the right hand side of Eq. (E4) becomes a sum of squares of polynomial of Hermitian operators and hence $n\left(4 \cos ^{2} \frac{\pi}{2 n}-1\right) \hat{\mathbf{1}}-\hat{\mathcal{B}}_{\mathrm{NLOS}}^{[n]} \geq 0$. As a result, the maximal quantum mechanical expectation value of $\hat{\mathcal{B}}_{\mathrm{NLOS}}^{[n]}$ is upper bounded by $n\left(4 \cos ^{2} \frac{\pi}{2 n}-1\right)$, so is the maximal value of $\mathcal{S}_{n}$ allowed in quantum theory.

Equivalently, it follows from Eq. (E2) that in quantum theory, the maximal winning probability $R^{n}$ is upper bounded by:

$$
\frac{1}{2}+\frac{1}{6 n} \times n\left(4 \cos ^{2} \frac{\pi}{2 n}-1\right)=\frac{1}{3}+\frac{2}{3} \cos ^{2} \frac{\pi}{2 n} .
$$

which is just $R_{n}^{\text {quantum }}$ given in Eq. (46).

\section{Appendix F: Necessary and sufficient conditions for joint measurability of noisy spin observables}

Theorem 13. Consider a set of noisy spin observables along the axes $\hat{n}_{k}$, that is, a set of POVMS $\left\{E_{X_{k}}^{k}\right\}$ with $X_{k} \in\{+1,-1\}$ of the form

$$
E_{X_{k}}^{k}=\frac{1}{2} \hat{\mathbf{1}}+\frac{1}{2} \vec{\sigma} \cdot X_{k} \eta \hat{n}_{k} .
$$

Defining $2^{N}$ different 3-vectors

$$
\vec{m}_{X_{1} \ldots X_{N}} \equiv \sum_{k=1}^{N} X_{k} \hat{n}_{k},
$$

a necessary condition for the spin observables to be jointly measurable is that

$$
\eta \leq \frac{1}{N} \frac{\sum_{X_{1} \ldots X_{N}}\left|\vec{m}_{X_{1} \ldots X_{N}}\right|^{2}}{\sum_{X_{1} \ldots X_{N}}\left|\vec{m}_{X_{1} \ldots X_{N}}\right|}
$$

and a sufficient condition is that

$$
\eta \leq \frac{2^{N}}{\sum_{X_{1} \ldots X_{N}}\left|\vec{m}_{X_{1} \ldots X_{N}}\right|} .
$$

Proof. Clearly,

$$
\eta=\operatorname{Tr}\left[\left(\vec{\sigma} \cdot X_{k} \hat{n}_{k}\right) E_{X_{k}}^{k}\right] .
$$

But given that this equality holds for both values of $X_{k}$ and for all $k$, we have

$$
\eta=\frac{1}{2 N} \sum_{k=1}^{N} \sum_{X_{k}} \operatorname{Tr}\left[\left(\vec{\sigma} \cdot X_{k} \hat{n}_{k}\right) E_{X_{k}}^{k}\right] .
$$

Recall that joint measurability of POVMs $\left\{E_{X_{k}}^{k}\right\}$ for different $k$ implies the existence of another POVM $\left\{E_{X_{1} X_{2} \ldots X_{N}}\right\}$ such that

$$
E_{X_{k}}^{k}=\sum_{X_{1} \ldots X_{N}, \text { fix } X_{k}} E_{X_{1} \ldots X_{N}}
$$

Consequently,

$$
\eta=\frac{1}{2 N} \sum_{X_{1} \ldots X_{N}} \operatorname{Tr}\left[\left(\vec{\sigma} \cdot \sum_{k=1}^{N} X_{k} \hat{n}_{k}\right) E_{X_{1} \ldots X_{N}}\right] .
$$


Defining $\vec{m}_{X_{1} \ldots X_{N}}$ as above, we may write $\eta$ as

$$
\eta=\frac{1}{2 N} \sum_{X_{1} \ldots X_{N}}\left|\vec{m}_{X_{1} \ldots X_{N}}\right| \operatorname{Tr}\left[\left(\vec{\sigma} \cdot \hat{m}_{X_{1} \ldots X_{N}}\right) E_{X_{1} \ldots X_{N}}\right] .
$$

where $\hat{m}_{X_{1} \ldots X_{N}}=\vec{m}_{X_{1} \ldots X_{N}} /\left|\vec{m}_{X_{1} \ldots X_{N}}\right|$. We then note that

$$
\operatorname{Tr}\left[\left(\vec{\sigma} \cdot \hat{m}_{X_{1} \ldots X_{N}}\right) E_{X_{1} \ldots X_{N}}\right] \leq \operatorname{Tr}\left[E_{X_{1} \ldots X_{N}}\right]
$$

to obtain the inequality

$$
\eta \leq \frac{1}{2 N} \sum_{X_{1} \ldots X_{N}}\left|\vec{m}_{X_{1} \ldots X_{N}}\right| \operatorname{Tr}\left[E_{X_{1} \ldots X_{N}}\right]
$$

We need only determine the maximum value of the righthand-side in a variation over all POVMs $\left\{E_{X_{1} \ldots X_{N}}\right\}$. Given that $\sum_{X_{1} \ldots X_{N}} E_{X_{1} \ldots X_{N}}=\hat{\mathbf{1}}$, we know that $\sum_{X_{1} \ldots X_{N}} \operatorname{Tr}\left[E_{X_{1} \ldots X_{N}}\right]=2$. Consequently, the $2^{N_{-}}$ dimensional vector $\left(\frac{1}{2} \operatorname{Tr}\left[E_{X_{1} \ldots X_{N}}\right]\right)_{X_{1} \ldots X_{N}}$ has unit 1norm. Thinking of $\sum_{X_{1} \ldots X_{N}}\left|\vec{m}_{X_{1} \ldots X_{N}}\right| \operatorname{Tr}\left[E_{X_{1} \ldots X_{N}}\right]$ as a scalar product, we see that it is maximized by taking $\left(\frac{1}{2} \operatorname{Tr}\left[E_{X_{1} \ldots X_{N}}\right]\right)_{X_{1} \ldots X_{N}}$ to be a unit vector parallel to $\left(\left|\vec{m}_{X_{1} \ldots X_{N}}\right|\right)_{X_{1} \ldots X_{N}}$ with unit 1-norm, that is,

$$
\frac{1}{2} \operatorname{Tr}\left[E_{X_{1} \ldots X_{N}}\right]=\frac{\left|\vec{m}_{X_{1} \ldots X_{N}}\right|}{\sum_{X_{1}^{\prime} \ldots X_{N}^{\prime}}\left|\vec{m}_{X_{1}^{\prime} \ldots X_{N}^{\prime}}\right|}
$$

which yields the necessary condition on $\eta$, Eq. (F3).

To derive the sufficient condition, Eq. (F4), we construct a POVM that jointly measures a set of spin observables with value of $\eta$ saturating the inequality. Any set of observables with smaller $\eta$ can then be jointly measured by simply adding uniformly random noise to this POVM.

The simulating POVM is

$E_{X_{1} \ldots X_{N}}=\frac{2\left|\vec{m}_{X_{1} \ldots X_{N}}\right|}{\sum_{X_{1}^{\prime} \ldots X_{N}^{\prime}}\left|\vec{m}_{X_{1}^{\prime} \ldots X_{N}^{\prime}}\right|}\left[\frac{1}{2} \hat{\mathbf{1}}+\frac{1}{2} \vec{\sigma} \cdot \hat{m}_{X_{1} \ldots X_{N}}\right]$

It suffices to demonstrate that this is indeed a POVM and that it coarse-grains to the appropriate noisy spin observables. First, note that

$$
\begin{aligned}
\sum_{X_{1} \ldots X_{N}} \vec{m}_{X_{1} \ldots X_{N}} & =\sum_{X_{1} \ldots X_{N}}\left(\sum_{j=1}^{N} X_{j} \hat{n}_{j}\right) \\
& =\sum_{j=1}^{N} \hat{n}_{j}\left(\sum_{X_{1} \ldots X_{N}} X_{j}\right) \\
& =0 .
\end{aligned}
$$

while

$$
\begin{aligned}
\sum_{\left\{X_{i}\right\}_{i \neq k}} \vec{m}_{X_{1} \ldots X_{N}} & =\sum_{\left\{X_{i}\right\}_{i \neq k}}\left(\sum_{j=1}^{N} X_{j} \hat{n}_{j}\right) \\
& =\sum_{j=1}^{N} \hat{n}_{j}\left(\sum_{\left\{X_{i}\right\}_{i \neq k}} X_{j}\right) \\
& =\hat{n}_{k} 2^{N-1} X_{k} .
\end{aligned}
$$

Also, note that because $\vec{m}_{X_{1} \ldots X_{k} \ldots X_{N}}=$ $-\vec{m}_{-X_{1} \cdots-X_{k} \cdots-X_{N}}$, it follows that $\left|\vec{m}_{X_{1} \ldots X_{N}}\right|=$ $\left|\vec{m}_{-X_{1} \cdots-X_{N}}\right|$ and consequently that

$$
\sum_{\left\{X_{i}\right\}_{i \neq k}}\left|\vec{m}_{X_{1} \ldots X_{N}}\right|=\frac{1}{2} \sum_{X_{1} \ldots X_{N}}\left|\vec{m}_{X_{1} \ldots X_{N}}\right| .
$$

It is then easy to verify that

$$
\begin{aligned}
\sum_{X_{1} \ldots X_{N}} E_{X_{1} \ldots X_{N}} & =\sum_{X_{1} \ldots X_{N}} \frac{2\left|\vec{m}_{X_{1} \ldots X_{N}}\right|}{\sum_{X_{1}^{\prime} \ldots X_{N}^{\prime}}\left|\vec{m}_{X_{1}^{\prime} \ldots X_{N}^{\prime}}\right|} \\
& \times\left[\frac{1}{2} \hat{\mathbf{1}}+\frac{1}{2} \vec{\sigma} \cdot \hat{m}_{X_{1} \ldots X_{N}}\right] \\
& =\hat{\mathbf{1}}
\end{aligned}
$$

and that

$$
\begin{aligned}
\sum_{\left\{X_{i}\right\}_{i \neq k}} E_{X_{1} \ldots X_{N}} & =\sum_{\left\{X_{i}\right\}_{i \neq k}} \frac{2\left|\vec{m}_{X_{1} \ldots X_{N}}\right|}{\sum_{X_{1}^{\prime} \ldots X_{N}^{\prime}}\left|\vec{m}_{X_{1}^{\prime} \ldots X_{N}^{\prime}}\right|} \\
& \times\left[\frac{1}{2} \hat{\mathbf{1}}+\frac{1}{2} \vec{\sigma} \cdot \hat{m}_{X_{1} \ldots X_{N}}\right] \\
& =\frac{1}{2} \hat{\mathbf{1}}+\frac{1}{2} \vec{\sigma} \cdot \frac{2^{N}}{\sum_{X_{1}^{\prime} \ldots X_{N}^{\prime}}\left|\vec{m}_{X_{1}^{\prime} \ldots X_{N}^{\prime}}\right|} X_{k} \hat{n}_{k} .
\end{aligned}
$$

This establishes the sufficient condition.

Corollary 14. The necessary and sufficient conditions for joint measurability of a set of spin observables are: for a pair of orthogonal spin axes,

$$
\eta \leq \frac{1}{\sqrt{2}}
$$

for a triple of orthogonal spin axes,

$$
\eta \leq \frac{1}{\sqrt{3}}
$$

for a pair of trine spin axes,

$$
\eta \leq \sqrt{3}-1
$$

for a triple of trine spin axes,

$$
\eta \leq \frac{2}{3}
$$

To saturate each of these inequalities, it suffices to implement the POVM defined in Eq. (F13). 
Proof. We begin by establishing the values of $\left\{\left|\vec{m}_{X_{1} \ldots X_{N}}\right|\right\}$ for each of our four examples. For orthogonal spin axes, defined in Eq. (94), we have for $N=2$,

$$
\forall X_{1}, X_{2}:\left|\vec{m}_{X_{1} X_{2}}\right|=\left|X_{1} \hat{n}_{1}+X_{2} \hat{n}_{2}\right|=\sqrt{2},
$$

and for $N=3$,

$$
\begin{aligned}
\forall X_{1}, X_{2}, X_{3}:\left|\vec{m}_{X_{1} X_{2} X_{3}}\right| \\
=\left|X_{1} \hat{n}_{1}+X_{2} \hat{n}_{2}+X_{3} \hat{n}_{3}\right|=\sqrt{3} .
\end{aligned}
$$

For trine spin axes, defined in Eq. (100), we have for
$N=2$,

$$
\begin{aligned}
& \left|\vec{m}_{++}\right|=\left|\vec{m}_{--}\right|=1, \\
& \left|\vec{m}_{+-}\right|=\left|\vec{m}_{-+}\right|=\sqrt{3},
\end{aligned}
$$

and for $N=3$, we have (making use of the fact that $\hat{n}_{i}+\hat{n}_{j}=-\hat{n}_{k}$ for $i, j, k$ distinct),

$$
\begin{aligned}
\left|\vec{m}_{+++}\right| & =\left|\vec{m}_{---}\right|=0, \\
\left|\vec{m}_{++-}\right| & =\left|\vec{m}_{--+}\right|=\left|\vec{m}_{+-+}\right| \\
& =\left|\vec{m}_{-+-}\right|=\left|\vec{m}_{-++}\right|=\left|\vec{m}_{+--}\right|=2 .
\end{aligned}
$$

It is then straightforward to verify in each case that the necessary and sufficient conditions on $\eta$, Eqs. (F3) and (F4), coincide and yield the bounds given in Eqs. (F15)(F18). We have already shown that the bound of the sufficient condition is saturated by the POVM of Eq. (100).
[1] S. Kochen and E. P. Specker, J. Math. \& Mech. 17, 59 (1967).

[2] A. Cabello, quant-ph/0012089, Sec. I.E.

[3] A. Gleason, J. Math. \& Mech. 6, 885 (1957).

[4] J. S. Bell, Rev. Mod. Phys. 38, 447 (1966).

[5] Ernst Specker, "Die Logik nicht gleichzeitig entscheidbarer Aussagen," Dialectica 14, 239-246 (1960); English translation: "The logic of propositions which are not simultaneously decidable", Reprinted in C. A. Hooker (ed.), The Logico-Algebraic Approach to Quantum Mechanics. Volume I: Historical Evolution (Reidel, Dordrecht, 1975), pp. 135-140.

[6] J. S. Bell, Physics, 1, 195 (1964).

[7] M. Seevinck, arXiv:1103.4537 v1.

[8] A. Peres, Quantum Theory: Concepts and Methods (Kluwer, Dordrecht, 1993).

[9] A. A. Klyachko, M. A. Can, S. Binicioğlu, and A. S. Shumovsky, Phys. Rev. Lett. 101, 020403 (2008).

[10] N. D. Mermin, Phys. Today 38(4), 38 (1985).

[11] L. Hardy, Phys. Rev. Lett. 71, 1665 (1993).

[12] R. W. Spekkens, Phys. Rev. A 71, 052108 (2005).

[13] P. Busch, M. Grabowski, and P. J. Lahti, Operational quantum physics (Springer-Verlag, Berlin, 1995).

[14] T. Heinosaari, D. Reitzner, P. Stano, Found. Phys. 38, 1133 (2008).

[15] M. M. Wolf, D. Pérez-García, and C. Fernández, Phys. Rev. Lett. 103, 230402 (2009).

[16] S. Yu, N.-L. Liu, L. Li, and C. H. Oh, Phys. Rev. A 81, 062116 (2010).

[17] S. Popescu and P. Rohrlich, Found. Phys. 24, 379 (1994).

[18] R. W. Spekkens, Phys. Rev. A 75, 032110 (2007); arXiv:quant-ph/0401052

[19] A. Fine, Phys. Rev. Lett. 48, 291 (1982).

[20] N. D. Mermin, Rev. Mod. Phys., 65, 803 (1993).

[21] A. A. Klyachko, arXiv:quant-ph/0206012.

[22] O. Gühne, M. Kleinmann, A. Cabello, J.-A. Larsson, G. Kirchmair, F. Zähringer, R. Gerritsma, and C. F. Roos, Phys. Rev. A 81, 022121 (2010).
[23] M. Navascués, S. Pironio, and A. Acín, Phys. Rev. Lett. 98, 010401 (2007); idem, New J. Phys., 10, 073013 (2008); A. C. Doherty, Y.-C. Liang, B. Toner, and S. Wehner in Proceedings of the 23rd IEEE Conference on Computational Complexity (IEEE Computer Society, College Park, MD, 2008), pp. 199-210; idem, eprint arXiv:0803.4373 (2008); S. Pironio, M. Navascués, and A. Acín, SIAM J. Optim. 20, 2157 (2010).

[24] R. Clifton, Am. J. Phys. 61, 443 (1993).

[25] A. Cabello and G. García-Alcaine, J. Phys. A 29, 1025 (1996)

[26] H. S. M. Coxeter, Regular Polytopes, Dover Publications (1973).

[27] I. Pitowsky, Quantum Probability-Quantum Logic, (Springer, Berlin, 1989).

[28] D. Z. Albert, Y. Aharonov, and S. D'Amato, Phys. Rev. Lett. 54, 5 (1985).

[29] M. S. Leifer and R. W. Spekkens, Phys. Rev. Lett. 95, 200405 (2005).

[30] P. Badziçg, I. Bengtsson, A. Cabello, H. Granström, J.A. Larsson, Found. Phys. 41, 41 (2011).

[31] J. Barrett, N. Linden, S. Massar, S. Pironio, S. Popescu, and D. Roberts, Phys. Rev. A 71, 022101 (2005) .

[32] N. S. Jones and Ll. Masanes, Phys. Rev. A 72, 052312 (2005) .

[33] J. S. Bell, Speakable and Unspeakable in Quantum Mechanics (Cambridge University Press, Cambridge, 2004), Chapter 7 and Chapter 24.

[34] R. Cleve, P. Høyer, B. Toner, and J. Watrous, in Proceedings of the 19th IEEE Annual Conference on Computational Complexity 2004, Amherst, MA, (IEEE Conference Proceedings, New York, 2004), pp. 236-249.

[35] D. Avis, H. Imai, T. Ito, and Y. Sasaki, J. Phys. A 38, 10971 (2005).

[36] Ll. Masanes, Quantum Inf. Comput. 3, 345 (2003).

[37] D. Collins and N. Gisin, J. Phys. A 37, 1775 (2004).

[38] L. Vaidman, arXiv:quant-ph/0107057.

[39] S. Braunstein and C. M. Caves, Ann. Phys. 202, 22 
(1990).

[40] D. Boschi, S. Branca, F. De Martini, and L. Hardy, Phys. Rev. Lett. 79, 2755 (1997).

[41] H. P. Stapp, Mind, Matter, and Quantum Mechanics (Springer-Verlag, Berlin, 1993); Am. J. Phys. 65, 300 (1997).

[42] N. D. Mermin, arXiv:quant-ph/9711052 v1.

[43] W. Unruh, arXiv:quant-ph/9710032 v2.

[44] T. Fritz, arXiv:1006.2497v1 [quant-ph].

[45] K. Pál and T. Vértesi, Phys. Rev. A 82, 022116 (2010).

[46] H. Everett, III, Rev. Mod. Phys. 29, 454 (1957).

[47] P. Mitchell, S. Popescu, and D. Roberts, Phys. Rev. A 70, 060101(R) (2004).

[48] D. Collins, Ph.D. thesis, University of Bristol (2002), chap. 3.

[49] H.-J. Schmidt, arXiv:cond-mat/0604591 v1 [condmat.mtrl-sci]

[50] R. W. Spekkens, D. H. Buzacott, A. J. Keehn, B. Toner, G. J. Pryde, Phys. Rev. Lett. 102, 010401 (2009).

[51] J. Barrett, unpublished.

[52] D. J. Saunders, S. J. Jones, H. M. Wiseman, and G. J. Pryde, Nature Physics 6, 845 (2010).

[53] W. van Dam, e-print arXiv:quant-ph/0501159

[54] Ll. Masanes, A. Acín, and N. Gisin, Phys. Rev. A73, 012112 (2006).
[55] G. Brassard, H. Buhrman, N. Linden, A. A. Méthot, A. Tapp, and F. Unger, Phys. Rev. Lett. 96, 250401 (2006).

[56] N. Linden, S. Popescu, A. J. Short, and A. Winter, Phys. Rev. Lett. 99, 180502 (2007).

[57] N. Brunner and P. Skrzypczyk Phys. Rev. Lett. 102, 160403 (2009).

[58] M. Pawłowski, T. Paterek, D. Kaszlikowski, V. Scarani, A. Winter, and M. Żukowski, Nature 461, 1101 (2009).

[59] J. Allcock, N. Brunner, M. Pawłowski, and V. Scarani, Phys. Rev. A 80, 040103(R) (2009).

[60] M. Navascués and H. Wunderlich, Proc. Roy. Soc. Lond. A 466, 881 (2009)

[61] A. Acín, R. Augusiak, D. Cavalcanti, C. Hadley, J. K. Korbicz, M. Lewenstein, Ll. Masanes, and M. Piani, Phys. Rev. Lett. 104, 140404 (2010).

[62] J. Oppenheim and S. Wehner, Science 330, 1072 (2010).

[63] R. F. Werner and M. M. Wolf, Quant. Inf. Comput. 1 (3), 1 (2001).

[64] Y.-C. Liang and A. C. Doherty, Phys. Rev. A 75, 042103 (2007).

[65] S. L. Braunstein, A. Mann, and M. Revzen, Phys. Rev. Lett. 68, 3259 (1992).

[66] S. Wehner, Phys. Rev. A 73, 022110 (2006). 